\title{
Widespread regulation of gene expression in the Drosophila genome by the histone acetyltransferase dTip60
}

\author{
Inaugural-Dissertation \\ zur \\ Erlangung des Doktorgrades \\ Dr. rer. nat. \\ des Fachbereichs \\ Biologie und Geografie \\ an der \\ Universität Duisburg-Essen \\ vorgelegt von \\ Corinna Schirling \\ geboren in Heilbronn a. N.
}

Dezember 2008 
Die der vorliegenden Arbeit zugrunde liegenden Experimente wurden am Max-PlanckInstitut für Molekulare Genetik in Berlin und am Zentrum für Medizinische Biotechnologie (ZMB), Arbeitsgruppe Genetik an der Universität Duisburg-Essen durchgeführt.

1. Gutachter: Prof. Dr. Ann Ehrenhofer-Murray

2. Gutachter: Prof. Dr. Bernhard Horsthemke

3. Gutachter: Prof. Dr. Harald Saumweber, HU Berlin

Vorsitzender des Prüfungsausschusses: Prof. Dr. Daniel Hoffmann

Tag der mündlichen Prüfung: 23. März 2009 


\section{Table of contents}

Table of figures

List of tables

Abbrevations

$1 \quad$ Introduction 9

1.1 Chromatin organization 9

1.2 Histone modifications, histone variants and chromatin remodeling complexes 11

1.3 The MYST family of HATs 13

1.4 The MYST HAT dTip60 is part of a multiprotein complex 14

1.5 Some Tip60 complex components can have functions outside of the Tip60 complex 17

1.6 Genetic interactions of dTip60 18

1.7 Functions of Tip60 18

1.8 Aim of this work 20

$2 \quad$ Material and Methods 22

2.1 Organisms 22

2.1.1 E. coli strains _ 22

2.1.2 Drosophila stocks__ 22

2.2 Media and growth conditions _ 23

2.2.1 Media and growth conditions for E. coli__ 23

2.2.2 Cultivation of Schneider SL2 Zellen___ 23

2.2.3 Drosophila husbandry __ 23

2.3 Molecular cloning ___ 24

2.4 P-element transformation _ 26

$2.5 \quad$ P-element mobilization __ 26

2.6 Antibody generation _ 27

2.7 Preparation of cell extracts, SDS-PAGE and Western blot $\_28$

$2.8 \quad$ Immunohistochemistry _ 28

2.8.1 Whole-mount fluorescent staining of Drosophila embryos __ 28

2.8.2 In situ staining of Drosophila embryos ___ 29 
2.8.3 Fluorescent staining of polytene chromosomes 30

2.8.4 Staining of imaginal wing discs 30

2.9 dTip60 knock-down in SL2 cells 31

2.10 Genome-wide gene expression profiling 31

2.11 Quantitative real-time PCR 32

2.12 Chromatin Immunoprecipitation (ChIP) 34

2.13 Bioinformatics 34

3 Results 36

3.1 Phenotypes of dTip60 knock-down in Drosophila 36

3.1.1 Use of the Gal4/UAS system for RNA interference in flies 36

3.1.2 Strong knock-down of dTip60 led to lethality, whereas moderate knock-down caused developmental defects in the wing

3.1.3 Knock-down of dTip60 in the imaginal wing disc led to apoptosis and enrichment of H3K27me3 41

3.2 Generation of an antiserum specific for dTip60 42

3.3 Nuclear localization of dTip60 during embryonic development 43

3.4 dTip60 localized to interbands on polytene chromosomes 45

3.5 Genome-wide expression analysis revealed a major role for dTip60 in gene regulation 48

3.6 dTip60-regulated genes were involved in chromatin-related processes 50

3.7 dTip60 acted as a repressor at distinct genes 51

3.8 dTip60 was physically located at repressed genes 55

3.9 Cooperation of dTip60 and HDAC1 in gene repression 56

4 Discussion 59

4.1 dTip60 was essential for viability in flies 59

4.2 Effects of dTip60 on wing development 60

4.3 dTip60 in transcriptional activation 62

4.4 Function and localization of dTip60 on polytene chromosomes 63

4.5 dTip60 in transcriptional repression 63

4.5.1 dTip60 might mediate its repressive function by deposition of $\mathrm{H} 2 \mathrm{Av}$ 64

4.5.2 The repressive function of dTip60 might be independent of its HAT function 65

4.5.3 dTip60 might cooperate with HDAC1 in gene regulation 65 

70

6

Zusammenfassung 71

$7 \quad$ References 72

$8 \quad$ Danksagung 80

9 Lebenslauf 81

\section{Table of figures}

Figure 1 Chromatin forms higher structures............................................. 10

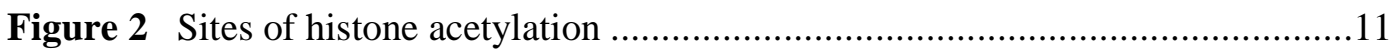

Figure 3 Phylogenetic tree of MYST histone acetyltransferases. ..........................14

Figure 4 Components of transcription-associated complexes that contain Pontin and Reptin. .17

Figure 5 Samples of purified recombinant fusion proteins dTip60-A and dTip60-B as used for generation of a dTip60-specific antiserum. .27

Figure 6 Mechanism of RNAi knock-down induced by the Gal4/UAS system. .37

Figure 7 Overview of dsRNA constructs used for construction of the UAS-Gal4 RNAi fly line (UAS sense and antisense) and RNAi in SL2 cells (ds1, ds2, ds3). .38

Figure 8 Downregulation of dTip60 by RNAi resulted in defects in the Drosophila wing.

Figure 9 Position, length and frequency of ectopic wing veins caused by dTip60RNAi driven by $\mathrm{T} 80$ or en.

Figure 10 Effects of en-Gal4-driven dTip60 downregulation in the imaginal wing disc.

Figure 11 Western blot analysis indicated that the antiserum raised against dTip60 recognized dTip60 protein..

Figure 12 Localization of the dTip60 protein in Drosophila embryos. .44

Figure 13 Ubiquitous expression of dTip60 RNA during embryogenesis. .45

Figure 14 dTip60 localized to interbands of polytene chromosomes.

Figure 15 dTip60 is reduced on polytene chromosomes of dTip60-RNAi animals. ...47 
Figure 16 Proliferation of SL2 cells was reduced after 5 days of RNAi treatment with dsRNA against dTip60 (ds1-3) and Aur, but not with mock-treatment against eGFP. ..48 Figure 17 After five days of RNAi treatment with ds3, dTip60 mRNA was visibly reduced in comparison to Actin mRNA levels.

Figure 18 Genome-wide expression analysis upon loss of dTip60. .50

Figure 19 Proliferation of treated (dTip60-RNAi) and mock-treated (eGFP-RNAi) cells. .52

Figure 20 Upregulation of selected genes in SL2 cells after zero to five days of dTip60 RNAi treatment. .53

Figure 21 Downregulation of selected genes and dTip60 expression in SL2 cells after zero to five days of dTip60 RNAi treatment. .54

Figure 22 Localization of dTip60 protein at dTip60-regulated genes by ChIP analysis. 


\section{List of tables}

Table 1 Components of the Tip60 complex in H. sapiens, D. melanogaster and $S$. cerevisiae. .16

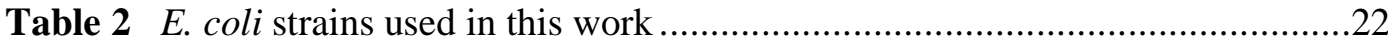

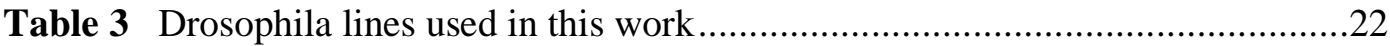

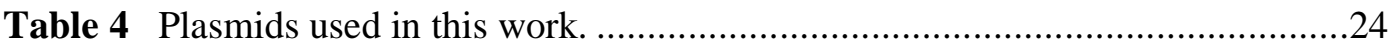

Table 5 Oligonucleotides used for molecular cloning, tagging and generation of

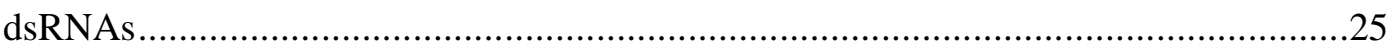

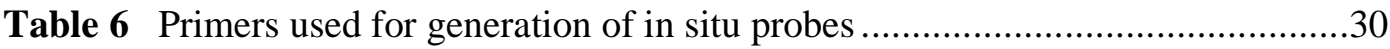

Table 7 Primers used for quantitative PCR (qPCR) in the body of the gene ...............33

Table 8 Primers used for qPCR at open reading frames (ORFs) ............................33

Table 9 Phenotypes of dTip60 RNAi knock-down in flies........................................39

Table 10 GO category association of genes regulated by dTip60 ...........................51

Table 11 Comparison of genes upregulated upon dTip60 depletion with TSA treatment

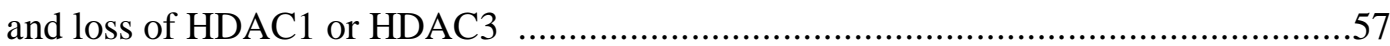

Table 12 Comparison of genes downregulated upon dTip60 depletion with TSA

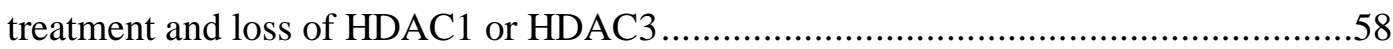




\section{Abbreviations}

aa

bp

ChIP

HAT

HDAC

$\mathrm{kDa}$

Mbp

ORF

PAGE

PCR

RNAi

rpm

SDS

SL2

wt amino acid

base pair

Chromatin immunoprecipitation

histone acetyltransferase

histone deacetylase

kilo dalton

mega base pairs

open reading frame

polyacrylamide gel electrophoresis

polymerase chain reaction

RNA interference

rounds per minute

sodium dodecyl sulfate

Schneider cells

wild type 


\section{Introduction}

\subsection{Chromatin organization}

In eukaryotes, the genome is organized into chromatin, which consists of histone proteins, chromatin-associated proteins and DNA. The basic repeat unit of chromatin is the nucleosome, within which 146 base pairs of DNA are wrapped around a histonoctamer composed of two copies each of the histones H2A, H2B, H3 and H4 (Kornberg, 1974; Luger et al., 1997). Not all, but most eukaryotes have another histone called H1, which serves as a linker histone (Godde and Ura, 2008). Chromatin forms higher order structures. "Beads on a string" refers to a structure in which non-condensed nucleosomes are repeated every $200 \pm 40 \mathrm{bp}$ and that is about $10 \mathrm{~nm}$ in width (Figure 1). The nucleosomal array is proposed to be further condensed in a $30 \mathrm{~nm}$ fiber, with six nucleosomes per turn. Higher-order chromatin folding is achieved with the help of superordinate proteins.

Chromatin compaction is necessary to adapt the size of the DNA molecule to nuclear dimensions. The Drosophila genome, for example, is about $180 \mathrm{Mbp}$ in size and has four chromosomes. Since one base pair corresponds to $3,4 \times 10^{-8}$ meters, a DNA molecule can be of considerable length. Chromatin compactions allows the structured packaging of DNA of several meters in length in few $\mu \mathrm{m}$. This is furthermore important to enable the correct distribution of chromosomes during mitosis.

On a global scale, chromatin is organized in heterochromatin and euchromatin. Heterochromatic domains display a closed chromatin conformation in comparison to euchromatic domains, where the chromatin structure is more open (Grewal and Moazed, 2003). This can be experimentally demonstrated by a nuclease assay: euchromatin is more accessible to nuclease than heterochromatin (Wallrath and Elgin, 1995). Furthermore, heterochromatin contains few actively transcribed genes, whereas euchromatin is rich in actively transcribed genes. Silencing in heterochromatin is established and maintained with the help of heterochromatic proteins that bind to nucleosomes, for example HP1 in Drosophila (McBryant et al., 2006).

A phenomenon called position effect variegation (PEV) in Drosophila illustrates the properties of eu- and heterochromatin (Ebert et al., 2006). Euchromatic genes, for example the white gene, which is responsible for the red eye colour of Drosophila, become silenced when their position is changed from eu- to heterochromatic regions. 
Although the sequence of the gene is unaltered, its expression is modified by its position within the chromatin. Phenotypically, the extent of silencing varies between the clonal populations that give rise to the insect compound eye, thus leading to a variegation in eye colour.

As a consequence of chromatin compaction, chromatin is generally repressive and has to be opened up to allow access for transcription, replication, recombination and repair (Ehrenhofer-Murray, 2004). In the next paragraphs, it will be discussed how dynamic changes in chromatin structure can be achieved.

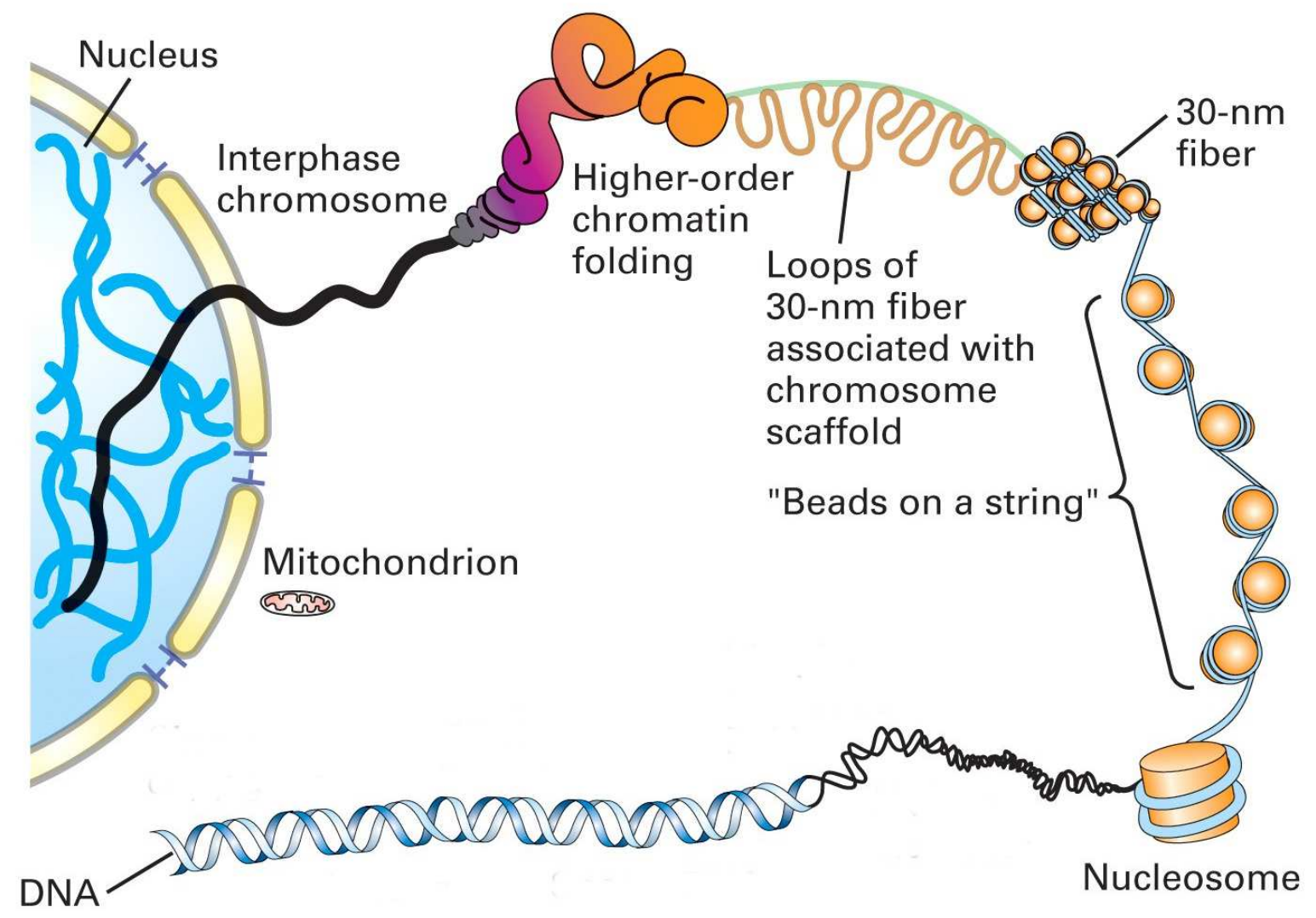

Figure 1: Chromatin forms higher structures. (Modified after Lodish, $5^{\text {th }}$ edition.) 


\subsection{Histone modifications, histone variants and chromatin remodeling complexes}

Chromatin structure can be altered by three mechanisms: histone modifications, histone variants and chromatin remodeling complexes.

Histones are small basic proteins and can carry posttranslational modifications, namely acetylation, methylation, phosphorylation, ubiquitylation, sumoylation and ADPribosylation (Ehrenhofer-Murray, 2004). The majority of these modifications are positioned at the $\mathrm{N}$-terminal tails of histones, with the exception of ubiquitylation, which is found at the C-terminal part of $\mathrm{H} 2 \mathrm{~A}$ and $\mathrm{H} 2 \mathrm{~B}$. However, it is becoming increasingly evident that histones can also carry posttranslational modifications in the core region (Zhang et al., 2003).

Histone acetylation is introduced by histone acetyltransferases (HATs) at the $\varepsilon-\mathrm{NH}_{3}{ }^{+}$ position of lysine residues of the N-terminal histone tail. An overview of the sites of histone acetylations on different histones is given in Figure 2.

H2A SGRGKQGGKARAKAKTRSSR
$1 \quad 5 \quad 9 \quad 20$

$\mathrm{H} 2 \mathrm{~B}$

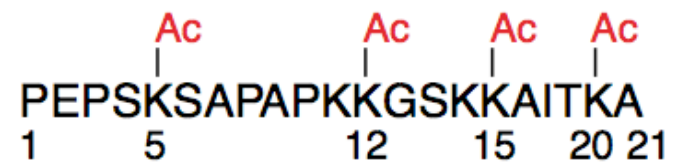

H3

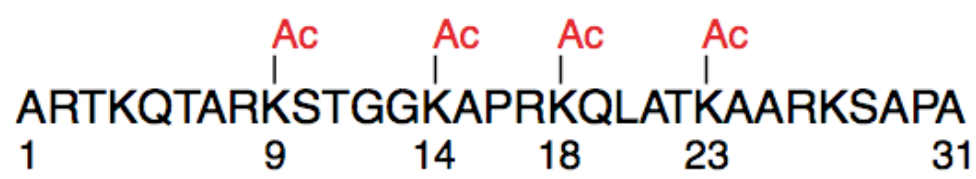

$\mathrm{H} 4$

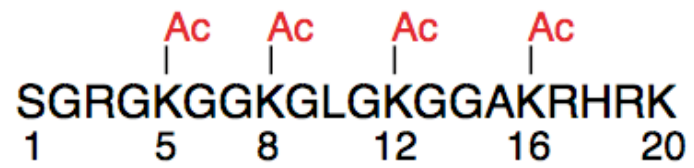

Figure 2: Sites of histone acetylation (Carrozza et al., 2003) 
Histone acetylation is reversible, with histone deacetylation being faciliated by histone deacetylases (HDACs). In euchromation, histone acetylation is enriched, whereas in heterochromatin, histones are hypoacetylated. Generally, histone acetylation is thought to open up chromatin structure by neutralizing histone charge, which weakens DNAhistone and nucleosome contact. This reduces chromatin compaction and faciliates access of DNA-binding factors (Workman and Kingston, 1998). The biological effects might also result from read-out of histone modifications by superordinate proteins. It is proposed that histone modifications form a histone code and can serve as a platform for recruitment of other multi-protein complexes with functions in transcriptional activation or repression (Turner, 2002).

Another way to modify chromatin structure is by incorporation of histone variants. Examples for histone variants are CenH3 (Cid in Drosophila, Cse4 in S. cerevisiae), which is enriched in centromeric regions, and variants of H2A, H2A.X and H2A.Z (Kamakaka and Biggins, 2005). In Drosophila, the properties of H2A.X and H2A.Z are unified in $\mathrm{H} 2 \mathrm{Av}$. When DNA double-strand breaks (DSB) occur, H2A.X/H2Av is phosphorylated at the site of DNA damage. This serves as a signal for the recruitment of the DSB repair machinery (Kinner et al., 2008). It has been demonstrated that H2Av localizes to centromeric heterochromatin (Swaminathan et al., 2005). Furthermore, there is evidence that the Remodeling and Spacing Factor RSF governs silent chromatin formation via replacement of H2Av (Hanai et al., 2008). Interestingly, a role for $\mathrm{H} 2 \mathrm{Av}$ in chromatin architecture has recently emerged: $\mathrm{H} 2 \mathrm{Av}$ is enriched at the 5' end of genes, and genes that possess $\mathrm{H} 2 \mathrm{Av}$ are likely to have experienced a transcription event (Mavrich et al., 2008).

Chromatin remodeling machines are essential for changes and dynamics of chromatin structure. These remodeling factors do not affect the biochemical properties of nucleosomes. Instead, they use the energy from ATP to slide nucleosomes along a particular DNA sequence, alter DNA-histone interactions, ease histone exchange or even enable complete removal of histones at a specific location (Becker and Horz, 2002). As chromatin remodeling factors need the energy from ATP-hydrolysis, they all have an ATPase subunit and therefore can be grouped according to the sequence of this subunit. The Swi2/Snf2 superfamily of chromatin remodelers is divided into four classes: SWI/SNF, ISWI, CHD and INO80. In yeast, the INO80 complex and the RSC complex, which belongs to SWI/SNF, can also function in DSB repair. 


\subsection{The MYST family of HATs}

HATs are divided into five families: Gen5-related HATs, p300/CBP HATs, the general transcription factor HATs, nuclear hormone receptor-related HATs and the MYST family of HATs (Carrozza et al., 2003). The MYST family is named for its founding members $\underline{\mathrm{MOZ}}, \underline{\mathrm{Y}} \mathrm{bf} 2 / \mathrm{Sas} 3, \underline{\mathrm{S}} \mathrm{as} 2$ and $\underline{\mathrm{Tip}} 60$. These proteins are grouped because of their sequence similarities in that they share a structurally similar catalytic HAT domain (Sanjuan and Marin, 2001). MYST HATs are involved in a wide range of cellular functions and are highly conserved from yeast to humans (Figure 3).

The MYST family can be further subdivided into three subgroups (Utley and Cote, 2003): The first contains proteins carrying a PHD finger (MOZ and MORF), the second contains a zinc finger motif (HBO1, Sas2), and the third, comprising Esa1, dMOF and Tip60, contains a chromodomain, which in dMOF has RNA-binding capacity (Akhtar et al., 2000).

In Drosophila, there are five MYST HATs. The properties of the MYST HAT dMOF have been well characterized. $\mathrm{dMOF}$ is a catalytically active subunit of the Dosage Compensation Complex (DCC) in Drosophila (Buscaino et al., 2003). In order to ensure equal expression of $\mathrm{X}$-chromosomal genes in male and female flies, the male $\mathrm{X}$ chromosome is hyperacetylated by dMOF, which is specific for H4K16 acetylation. Recent work demonstrated that MOF also has a function in gene regulation on autosomes (Kind et al., 2008).

Another MYST HAT in Drosophila, Enok, plays a role in mushroom body formation in the drosophila brain (Scott et al., 2001). The Drosophila homologue of HBO1, Chameau, is a dominant suppressor of position effect variegation and enhances silencing of Hox genes by the Polycomb group proteins. Interestingly, overexpression by Chameau partially rescues telomeric silencing in sas $2 \Delta$ knock-out mutants in $S$. cerevisiae, suggesting that Sas2 and Chameau might have equivalent functions (Grienenberger et al., 2002). Furthermore, there is evidence that Chameau and the histone deacetylase $\mathrm{dRpd} 3$ act as antagonistic cofactors of dJun and $\mathrm{dFos}$, modulating JNK-dependent transcription during metamorphosis in Drosophila (Miotto et al., 2006). The proposed Drosophila MYST HAT CG1894 is of unknown function and has not been further characterized. The properties of dTip60 will be discussed in detail in the following paragraphs. 


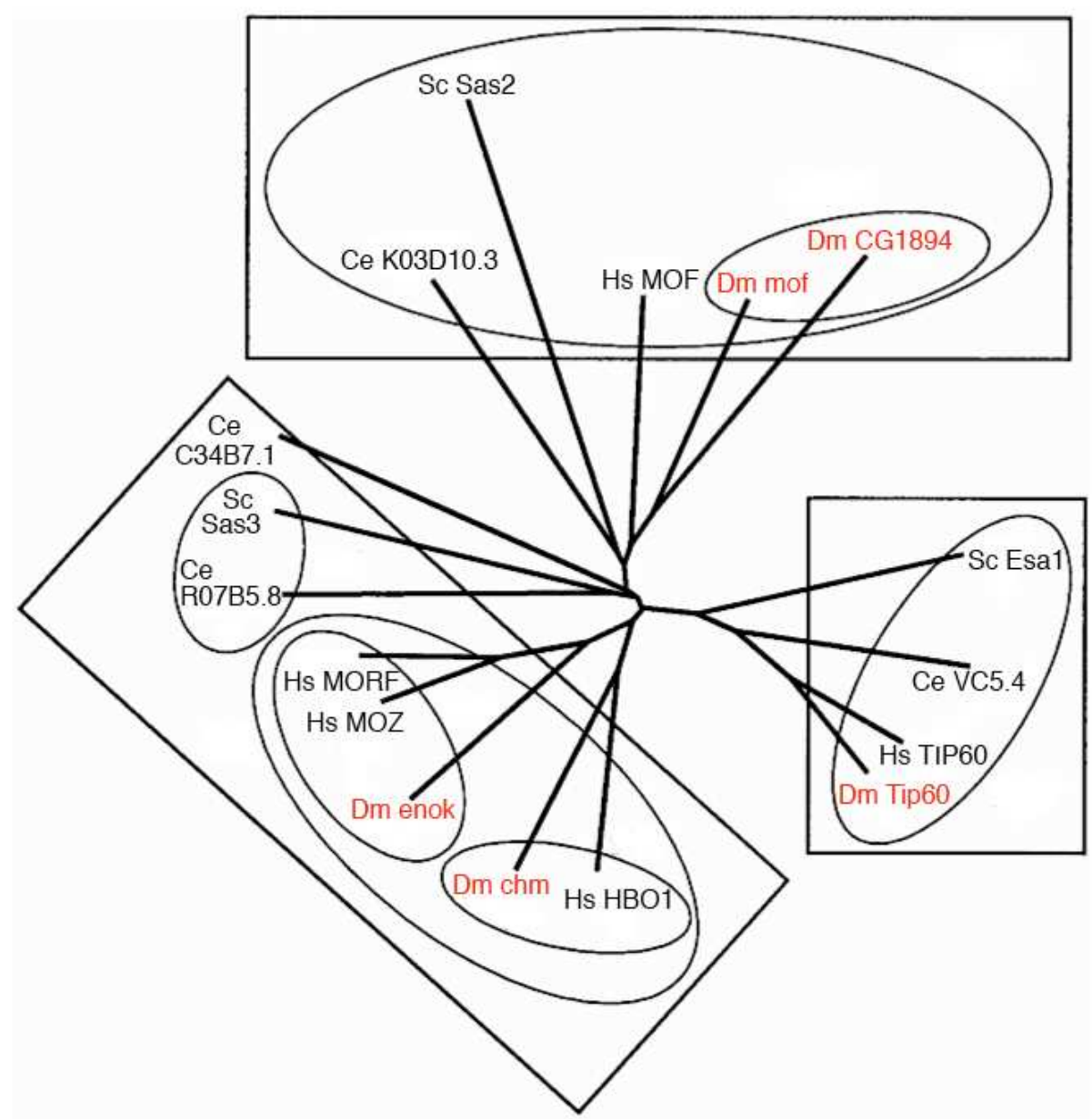

Figure 3: Phylogenetic tree of MYST histone acetyltransferases. (Sanjuan and Marin, 2001)

\subsection{The MYST HAT dTip60 is part of a multiprotein complex}

Tip60 was first identified as a protein interacting with the Tat protein from HIV, and hence was named Tat interactive protein, $60 \mathrm{kDa}$ (Kamine et al., 1996). Like many other HATs, Tip60 is part of a multiprotein complex (Table 1) whose basic composition is evolutionarily conserved (Doyon et al., 2004). Remarkably, the Tip60 complex in human and Drosophila unites two distinct chromatin-modifying activities, histone acetylation and chromatin remodeling (Sapountzi et al., 2006; Squatrito et al., 2006). These activities are found in two separate complexes in the yeast Saccharomyces cerevisiae, the HAT complex NuA4 and the ATP-dependent chromatin remodeler SWR1. 
In vitro, the Tip60 complex preferentially acetylates nucleosomal H2A and H4 (Ikura et al., 2000). Next to the Tip60 enzyme itself, the NuA4 part of the Tip60 complex contains the scaffolding protein TRRAP, actin and the actin-related protein BAF35a as well as several proteins containing motifs common to many chromatin proteins (Sapountzi et al., 2006). The proteins Mrg15 and $\operatorname{MrgX}$ contain a chromodomain, and ING3 contains a PHD finger, motifs that in some contexts recognize methylated histones. Furthermore, DMAP contains a SANT domain, and Gas41 a YEATS domain. Other components are Epc1 and Epc1-like, which are homologous to Drosophila E(Pc), and FLJ11730, which is homologous to S. cerevisiae Eaf6. All these components have equivalents in the respective fly and yeast complexes (Sapountzi et al., 2006). A similar complex has been identified genetically in C. elegans (Ceol and Horvitz, 2004).

The chromatin-remodeler portion of the Tip60 complex comprises p400/ Domino, which is a SWI2/ SNF2-like ATPase, and the RuvB-like helicase proteins RuvBL1 and RuvBL2 (Ikura et al., 2000), whose homologs in Drosophila are called dPontin and dReptin, respectively (Gallant, 2007). It further contains the bromodomain protein Brd8, which may bind acetylated histones, and YL-1, which is homologous to the yeast SWR1 component Vps72 (Sapountzi et al., 2006). Notably, the Tip60 complex from Drosophila (dTip60) co-purifies with H2B and the histone variant H2Av (Kusch et al., 2004). When incorporated into nucleosomes in vitro, phospho- $\mathrm{H} 2 \mathrm{Av}$ is the preferred substrate of the dTip60 complex and is acetylated at lysine 5 (K5). The dTip60 complex catalyzes replacement of acetylated phospho-H2Av by unmodified $\mathrm{H} 2 \mathrm{Av}$ via its chromatin remodeler moiety (Kusch et al., 2004). Thus, the dTip60 complex is involved in a concerted acetylation-remodeling reaction that may be required to reverse the phospho-H2Av mark imposed by DNA damage. In agreement with this, Tip60 complexes play a role in DNA damage repair in several organisms (Squatrito et al., 2006). More generally, this acetylation-remodeling activity may reflect the general activity of the Tip60 complex in transcriptional activation. 
Table 1: Components of the Tip60 complex in H. sapiens, D. melanogaster and S. cerevisiae. (Sapountzi et al., 2006). The asterisk (*) denotes subunits of the distinct SWR1 complex in yeast.

\begin{tabular}{|c|c|c|c|}
\hline $\begin{array}{l}\text { H. sapiens } \\
\text { complex }\end{array}$ & $\begin{array}{l}D . \\
\text { melanogaster } \\
\text { complex }\end{array}$ & $\begin{array}{l}\text { S. cerevisiae } \\
\text { complex (NuA4) }\end{array}$ & Proposed function \\
\hline TIP60 & dTip60 & Esa1 & Acetyltransferase \\
\hline TRRAP & dTra1 & Tra1 & PIKK domain \\
\hline Actin & Act87E & Act1 & ATPase, cytoskeleton \\
\hline BAF53a & BAP55 & Arp4 & Actin related, DNA repair \\
\hline P400/Domino & Domino & Eaf1/Swr1* & SWI2/SNF2-like ATPase \\
\hline RuvBL1 & dPontin & Rvb1* & \multirow[t]{2}{*}{ Helicase/ ATPase } \\
\hline RuvBL2 & dReptin & Rvb2* & \\
\hline $\operatorname{Mrg} 15$ & dMrg15 & \multirow[t]{2}{*}{ Eaf3 } & \multirow[t]{2}{*}{ Chromo domain, senescence } \\
\hline MrgX & & & \\
\hline MrgBP & dMrgBP & Eaf7 & \\
\hline Epc1 & $\mathrm{E}(\mathrm{Pc})$ & Epl1 & $\begin{array}{l}\text { Transcription control, } \\
\text { silencing }\end{array}$ \\
\hline Epc-like Protein & & & Transcription regulation \\
\hline ING3 & dIng3 & Yng2 & $\begin{array}{l}\text { PHD finger domain, growth } \\
\text { inhibitor, apoptosis }\end{array}$ \\
\hline Brd8/ TRCp120 & $\mathrm{dBrd} 8$ & Bdf1* & Bromo domain \\
\hline YL-1 & dYL-1 & Yps72* & Chromatin remodeling \\
\hline DMAP & dDMAP1 & Eaf2 & $\begin{array}{l}\text { SANT Domain, DNA } \\
\text { replikation }\end{array}$ \\
\hline Gas41 & dGas41 & Yaf9 & YEATS Domain, cell viability \\
\hline FLJ11730 & dEaf6 & Eaf6 & \\
\hline $\begin{array}{l}\text { (H2A.X/ } \\
\text { H2A.Z) }\end{array}$ & $\mathrm{H} 2 \mathrm{Av}$ & $\mathrm{H} 2 \mathrm{~A} / \mathrm{H} 2 \mathrm{~A} . \mathrm{Z}^{*}$ & \multirow[t]{2}{*}{ Histone } \\
\hline$(\mathrm{H} 2 \mathrm{~B})$ & $\mathrm{H} 2 \mathrm{~B}$ & $\mathrm{H} 2 \mathrm{~B} *$ & \\
\hline
\end{tabular}




\subsection{Some Tip60 complex components can have functions outside of the Tip60 complex}

Several of the Tip60 complex subunits are part of other complexes or have functions independent of Tip60. Pontin and Reptin are associated with other complexes (Figure 4), for example the Ino80 chromatin remodeling complex and the Uri complex (Gallant, 2007). The Uri complex probably contributes to the control of gene expression via the E3-ubiquitin ligase $\mathrm{SCF}^{\mathrm{Skp} 2}$ and its association with RNA polymerase II and the Paf complex (Adelman et al., 2006). Furthermore, Reptin in Drosophila is a component of the PRC1 complex (Qi et al., 2006), whereas Pontin purifies with the Brahma chromatin remodeling complex (Diop et al., 2008). Reporter gene assays demonstrated that Pontin and Reption antagonistically influence the transactivation potential of the B-cateninTCF complex, which is central to Wingless signaling (Bauer et al., 2000). Their residence in several complexes may explain these antagonistic effects. Also, the scaffolding protein TRRAP is present in several other HAT complexes, notably several Gcn5-containing complexes (Robert et al., 2006).

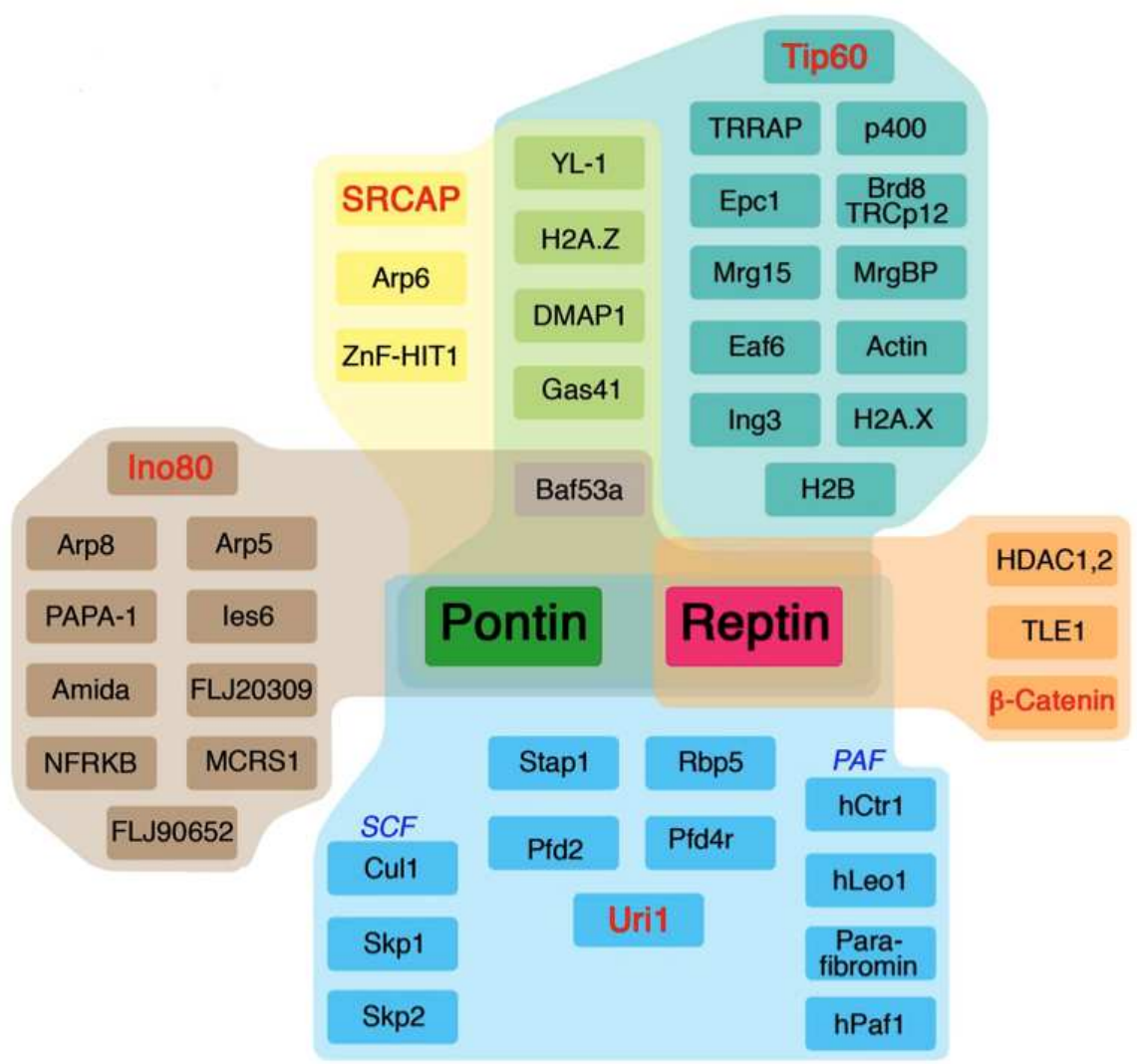

Figure 4: Components of transcription-associated complexes that contain Pontin and Reptin. (Source: (Gallant, 2007) 


\subsection{Genetic interactions of dTip60}

Recently, consequences of mutations of Reptin and its role in association with the dTip60 complex have been evaluated in flies (Qi et al., 2006). A P-element insertion in the Drosophila Reptin gene led to dramatic reduction of Reptin mRNA levels. Genetically, this Reptin mutant interacted with mutants of Polycomb Group (PcG) genes in a way that indicated that Reptin and Polycomb interact in the same pathway in vivo. Furthermore, the study demonstrated that Reptin is a suppressor of position-effect variegation and contributes to the generation of silent chromatin at many loci in Drosophila. Since Reptin has coresidency in more than one protein complex and can function independently of the dTip60 complex, the authors asked whether the phenotype and the proposed function of Reptin are dependent on other dTip60 complex components. It was shown that P-element-based mutations in the dTip60 complex components Enhancer of Polycomb, Domino and dMRG15 led to identical phenotypes as mutations in Reptin. This suggests that Reptin as a part of the dTip60 complex promotes the generation of silent chromatin.

Other work in Drosophila showed that dTip60 is essential on a cellular and multicellular level (Zhu et al., 2007). Transient transfection of SL2 cells with a vector that caused dTip60-RNAi led to morphological defects and lethality. On an organismal level, strong knock-down of dTip60 with the UAS/Gal4 system was reported to lead to lethality in the pupal stage. Further characterization of dTip60 expression levels revealed that it is differentially expressed during development: dTip60 mRNA levels peak at embryogenesis and are relatively low in $2^{\text {nd }}$ instar. In the adult fly, dTip60 mRNA levels peak again. Taken together, these findings highlight the importance of dTip60 for viability in Drosophila melanogaster.

\subsection{Functions of Tip60}

Among the many roles of histone acetyltransferases, gene activation is probably the most widely recognized. HATs are generally thought to activate gene transcription by loosening chromatin compaction and by creating binding sites for the bromodomain moieties of transcription factor complexes through lysine acetylation, therefore facilitating access of the transcriptional machinery to the DNA (Ehrenhofer-Murray, 2004). In agreement with this notion, the Tip60 complex is recruited to promoters by 
DNA-binding factors and acts as a transcriptional co-activator in several contexts. For example, Tip60 serves as a co-activator for nuclear hormone receptors (Gaughan et al., 2001), c-myc (Patel et al., 2004) and NF-KB (Baek et al., 2002). The co-activator function in some instances is mediated by histone acetylation in the promoter region (Taubert et al., 2004), whereas in other cases, Tip60 acetylates the transcription factor itself and thus modulates its activity, resulting in context-dependent transcriptional outcomes (Sapountzi et al., 2006). As an example, Tip60 acetylates p53, which helps to distinguish between the cell-cycle arrest and apoptotic functions of p53 (Legube et al., 2004; Sykes et al., 2006; Tang et al., 2006).

Interestingly, Tip60 is not exclusively involved in gene activation, but also has a role in gene regulation as a co-repressor. While this may not require its HAT activity, Tip60 interaction with the DNA binding factors STAT3 and KLF4 serves to recruit the histone deacetylase HDAC7 to gene promoters, which causes gene repression (Ai et al., 2007; Xiao et al., 2003).

The cooperation of Tip60 with some transcription factors seems to be highly context dependent in that it serves as a co-activator in some cases, whereas in others it is a transcriptional repressor. Myc recruits Tip60 to chromatin via the TRRAP subunit (Frank et al., 2003) and interacts with Pontin, thus regulating cellular growth and proliferation by gene repression (Bellosta et al., 2005). Moreover, Tip60 not only interacts with myc, but can also counteract myc as a haplo-insufficient tumour suppressor by modulating oncogene-induced DNA-damage response during lymphomagenesis in both mouse and humans (Gorrini et al., 2007). These findings imply complex, context-dependent roles of the Tip60 complex in transcription, precluding a simple prediction for Tip60 function.

On a physiological level, there are indications that Tip60 might be involved in Alzheimers disease. Tip60 forms a complex with AICD, which is a cleavage product of the amyloid- 3 precurser protein APP (Cao and Sudhof, 2001). Tip60, AICD and the transcriptional protein Fe65 stimulate acetylation and co-activate genes that are linked to apoptosis and neurotoxicity (Kinoshita et al., 2002). However, in other cases, Tip60 is not necessary for this transcriptional activation and can serve as a co-repressor for Fe65 (Sumioka et al., 2005).

Interestingly, there are two more examples indicating the formation of a complex between Tip60, its complex components and proteolytically cleaved intracellular domains of transmembrane proteins, one interaction partner being the low density 
lipoprotein receptor related protein (Kinoshita et al., 2003), the other being the Notch intracellular domain NICD (Gause et al., 2006). The cooperation between Tip60 and NICD might be mediated by the subunit TRRAP, and there is evidence that another HAT, dGCN5, is recruited (Gause et al., 2006). Again, Tip60 function is highly context-dependent, since other data indicate that Tip60 inhibits Notch signaling (Kim et al., 2007).

As a result of its roles in transcriptional regulation and double-strand break repair, Tip60 is also involved in several physiological processes on an organismal level. In $C$. elegans, a genetically defined Tip60 complex regulates Ras-mediated vulval induction in cooperation with a chromatin remodeling complex carrying histone deacetylase activity (Ceol and Horvitz, 2004). In Drosophila, the dTip60 complex components Reptin, Domino, Mrg15 and Epc1 promote the generation of silent chromatin in that mutations in these genes suppress position-effect variegation (Qi et al., 2006).

\subsection{Aim of this work}

The aim of this work was to gain insight into the properties of dTip60 in flies and its function in gene regulation. First, we were interested in the consequences of dTip60 reduction in flies. To this end, we constructed a dTip60 knock-down line in flies using the Gal4/UAS system. We found that a strong knock-down of dTip60 was lethal, whereas a moderate modulation of dTip60 protein levels led to the formation of ectopic veins in the wing. Next, we evaluated the function of dTip60 in imaginal wing discs. RNAi knock-down of dTip60 in imaginal wing discs led to an increased H3K27me3 and increased apoptosis. We further characterized the localization of dTip60 and found that in Drosophila embryos, dTip60 was located in the nucleus. On polytene chromosomes, dTip60 localized to regions within the interbands.

In order to investigate the role of dTip60 in gene regulation, we performed genome wide transcription analysis on dTip60-RNAi treated SL2 cells. dTip60 significantly regulated approximately $10 \%$ of all genes in Drosophila, which illustrates its physiological importance. We asked whether genes with defined biological functions were enriched among those regulated by dTip60, and found that indeed, chromatinrelated functions were highly enriched. The effect of dTip60 was validated for a subset of genes using qPCR. Surprisingly, our findings suggest that the histone acetyltransferase dTip60 has repressive as well as activating function. This raised the 
question whether this regulation was directly or indirectly mediated by dTip60. We performed chromatin immunopurification and found that dTip60 protein was directly located at promotor regions of repressed genes, suggesting a direct role in gene repression.

We hypothesized that dTip60 might act in cooperation with an HDAC. Comparison of the gene expression signature of dTip60 with that of HDACs revealed a significant correlation with the expression signature of HDAC1. This suggests that the dTip60 complex might recruit an HDAC1-containing complex to regulate gene expression in the Drosophila genome. 


\section{Material and Methods}

\subsection{Organisms}

\subsubsection{E. coli strains}

Table 2: E. coli strains used in this work

\begin{tabular}{|c|c|}
\hline Name & Description \\
\hline TOP10 & $\begin{array}{l}\text { F mcrA } \Delta \text { (mrr-hsdRMS-mcrBC) } \varphi 801 \text { lacZ } \Delta \text { M15 } \Delta \text { lacX74 recA1 } \\
\text { ara } \Delta 139 \Delta \text { (ara-leu) } 7697 \text { galU galK rpsL (StrR) endA1 nupG } \\
\text { (chemically or electro-competent; Invitrogen) }\end{array}$ \\
\hline DH5 $\alpha$ & 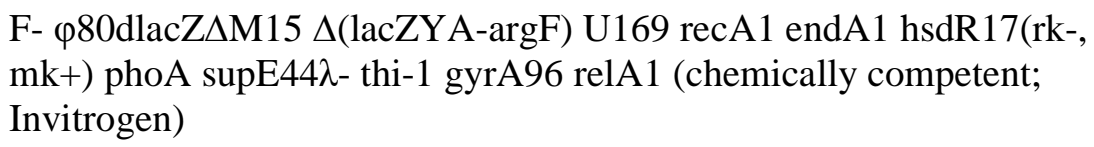 \\
\hline BL21 (DE3) & $\begin{array}{l}\text { F- omp T } h s d \mathrm{~S}\left(\mathrm{r}_{\mathrm{B}}^{-} \mathrm{m}_{\mathrm{B}}^{-}\right), d c m^{+} \mathrm{Tet}^{\mathrm{r}} \text { gal } \lambda(\mathrm{DE} 3) \text { endA Hte }[\arg U \text { ileY } \\
\left.\text { leuW } \mathrm{Cam}^{\mathrm{r}}\right] .\end{array}$ \\
\hline
\end{tabular}

\subsubsection{Drosophila stocks}

Table 3: Drosophila lines used in this work

\begin{tabular}{|l|l|}
\hline Fly line & Description \\
\hline Oregon R & Wild-type \\
\hline Da-Gal4 & Homozygous, located on 2nd chromosome \\
\hline T80-Gal4/CyO & Heterozygous, balanced over CyO, located on 2nd chromosome \\
\hline En-Gal4 & Homozygous, located on 2nd chromosome \\
\hline $\mathrm{w}^{1118}$ & Hemizygous \\
\hline dTip60-RNAi & Hemizygous \\
\hline
\end{tabular}

All fly strains were obtained from the laboratory of Prof. Saumweber (HU Berlin) with the exception of dTip60-RNAi, which was generated for this work (see 2.4). Further lines of dTip60-RNAi were obtained by P-element mobilization (see $\mathbf{2 . 5}$ ) 


\subsection{Media and growth conditions}

\subsubsection{Media and growth conditions for $E$. coli}

E. coli strains used for plasmid amplification and expression of recombinant protein were cultured according to standard procedures (Sambrook et al., 1989) at $37^{\circ} \mathrm{C}$ in Luria-Bertani (LB) medium supplemented with either $100 \mu \mathrm{g} / \mathrm{ml}$ ampicillin oder 50 $\mu \mathrm{g} / \mathrm{ml}$ kanamycin. For transformation, SOC medium was used. For plates, $20 \mathrm{~g} / \mathrm{l}$ agar was added to liquid media.

LB $10 \mathrm{~g} / \mathrm{l}$ caseinpeptone, $5 \mathrm{~g} / \mathrm{l}$ yeast extract, $5 \mathrm{~g} / \mathrm{l} \mathrm{NaCl}$

SOC $2 \mathrm{~g} / \mathrm{l}$ tryptone, $500 \mathrm{mg} / \mathrm{l}$ yeast extract, $10 \mathrm{mM} \mathrm{NaCl}, 2.5 \mathrm{mM} \mathrm{KCl}$, $10 \mathrm{mM} \mathrm{MgCl} 2,10 \mathrm{mM} \mathrm{MgSO} 4,20 \mathrm{mM}$ glucose

\subsubsection{Cultivation of Schneider SL2 Zellen}

Schneider cells (SL2) were derived from a primary culture of Drosophila embryos aged 20-24h (Schneider, 1972). Cells were cultured at $26^{\circ} \mathrm{C}$ in InsectExpress Sf9-S2 Medium (PAA). Unless noted otherwise, the medium was supplemented with $10 \%$ fetal calf serum (FCS, from PAA). Cells were split (1:1) at least once per week.

To assay cell viability and number, $50 \mu \mathrm{l}$ cells were mixed with $50 \mu \mathrm{l}$ trypan blue solution $(0,4 \%$; Sigma) and counted in a Neubauer counting chamber.

\subsubsection{Drosophila husbandry}

Fly strains were maintained in cylindric plastic vials with foam plugs and incubated at room temperature or as indicated. Instant Formula 24-4 (Carolina Biological Supply Company) was used as medium, and yeast was added to freshly prepared medium. 


\subsection{Molecular cloning}

Molecular cloning was performed according to standard procedures (Sambrook et al., 1989). Kits for plasmid purifications were purchased from Qiagen or Macherey-Nagel. Enzymes and respective buffers from NEB, Promega and Stratagene were used. Plasmids used in this work are listed in Table 4, oligonucleotides are listed in Table 5.

Table 4: Plasmids used in this work.

\begin{tabular}{|l|l|}
\hline Plasmid & Description \\
\hline pOT2- LD31064 & Contains full-length dTip60 cDNA; Open Biosystems \\
\hline pUAST & pUC Gal4 UAS; (Brand and Perrimon, 1993) \\
\hline pUAST-Tip60 RNAi & for details see Figure 7 \\
\hline pET15b & N-terminal His-Tag, Novagen \\
\hline pET15b-Tip60 A & His- tagged Tip60 fragment, AS 30 - 304 \\
\hline pET15b-Tip60 B & His- tagged Tip60 fragment, AS 305 - 541 \\
\hline pET15b-Tip60 full & His- tagged Tip60 fragment, AS 30 - 541 \\
\hline
\end{tabular}


Table 5: Oligonucleotides used for molecular cloning, tagging and generation of dsRNAs (fw $=$ forward, $\mathrm{rv}=$ reverse $)$

\begin{tabular}{|c|c|}
\hline Name & Sequence (5' to 3' direction) \\
\hline dTip60 sense fw & CCC GCG GCC GCA CAA GCG CTT GGA CGA GTG GG \\
\hline dTip60 sense rv & CGC CTC GAG GCC CTC GAA CTT GGA CAA CTC G \\
\hline dTip60 antisense $\mathrm{fw}$ & CGC GGT ACC GCG GAG AGA CGG CTC ACA AAC AGG \\
\hline dTip60 antisense rv & GCC CTC GAG GCC CTT GCG CTG ATA CGG AGG C \\
\hline dTip60 A fw & GCG CGC CAT ATG CCC GTC AGG ATG CAC AAA ACG \\
\hline dTip60 A rv & GCGCGGATCCTCTCACAGGCATTCCCTGCTCTTCTT \\
\hline dTip60 B fw & GCG CGC CAT ATG GAG AGG CAC CTG TCC AAG TGC \\
\hline dTip60 B rv & $\begin{array}{l}\text { GCG CGG ATC CTC TCA TTT GGA GCG CTT GGA CCA } \\
\text { GTC TTT GGG }\end{array}$ \\
\hline dTip60 full fw & dTip60 A fw \\
\hline dTip60 full rv & dTip60 B rv \\
\hline $\mathrm{ds} 1 \mathrm{fw}$ & $\begin{array}{l}\text { TTAATACGACTCACTATAGGGAGAAACAAGCGCTTGGA } \\
\text { CGAGTGG }\end{array}$ \\
\hline ds1 rv & $\begin{array}{l}\text { TTA ATA CGA CTC ACT ATA GGG AGA GCG ATG CCT } \\
\text { GCC CAG CTC GAT }\end{array}$ \\
\hline $\mathrm{ds} 2 \mathrm{fw}$ & $\begin{array}{l}\text { TA ATA CGA CTC ACT ATA GGG CCT ACA ACT CCG } \\
\text { CAG ACA G }\end{array}$ \\
\hline ds2 rv & $\begin{array}{l}\text { TA ATA CGA CTC ACT ATA GGG GAA CTC TGT CAT } \\
\text { TAC GTA GAA G }\end{array}$ \\
\hline $\mathrm{ds} 3 \mathrm{fw}$ & $\begin{array}{l}\text { TTA ATA CGA CTC ACT ATA GGG AGA TCC TAT CGA } \\
\text { TCG TAC TGG GC }\end{array}$ \\
\hline ds3 rv & $\begin{array}{l}\text { TTA ATA CGA CTC ACT ATA GGG AGA TCA TTT GGA } \\
\text { GCG CTT GGA CCA GTC }\end{array}$ \\
\hline Aur fw & $\begin{array}{l}\text { TAA TAC GAC TCA CTA TAG } \\
\text { GGATACGATTGGAGTCCCCGGGAC }\end{array}$ \\
\hline Aur rv & $\begin{array}{l}\text { TAA TAC GAC TCA CTA TAG } \\
\text { GGAGCTGTAGGTGCTCTCCGTGCTG }\end{array}$ \\
\hline eGFP fw & $\begin{array}{l}\text { TAA TAC GAC TCA CTA TAG GGA GAC CTG GTC GAG } \\
\text { CTG GAC GGC GAC }\end{array}$ \\
\hline eGFP rv & $\begin{array}{l}\text { TCT CCC TAT AGT GAG TCG TAT TAC GCG CTT CTC } \\
\text { GTT GGG GTC }\end{array}$ \\
\hline
\end{tabular}




\subsection{P-element transformation}

For dTip60-RNAi in flies, two dTip60 fragments were amplified from genomic DNA. The sense fragment spanned the base pairs 191-1222, while the antisense fragment spanned the base pairs 250-1275. The fragments were cloned in the P-element transformation vector pUAST (Brand and Perrimon, 1993) in sense and antisense orientation to generate the vector pUAST-Tip60-RNAi, which was injected into w1118 embryos to establish a homozygous transgenic line. Germline transformation was performed as described previously (Gortchakov et al., 2005).

\subsection{P-element mobilization}

As a genomic source of transposase for P-element mobilization, the strain $\mathrm{P}[\mathrm{ry}+\Delta 2-3] 99 \mathrm{~B}$ was used (Robertson et al., 1988).

F0:

$$
\frac{\mathrm{X}}{\mathrm{Y}} ; \frac{+}{+} ; \frac{\Delta 2-3, \mathrm{Ki}}{\mathrm{TM} 3, \mathrm{Sb}} \times \frac{\mathrm{P}\left[\mathrm{w}^{+} \text {Tip60-RNAi }\right]}{\mathrm{P}\left[\mathrm{w}^{+} \text {Tip60-RNAi }\right]} ; \frac{+}{+} ; \frac{+}{+}
$$

F1: $\frac{\mathrm{P}\left[\mathrm{w}^{+} \text {Tip60-RNAi }\right]}{\mathrm{Y}} ; \frac{+}{+} ; \frac{\Delta 2-3, \mathrm{Ki}}{+} \times \frac{\mathrm{w}}{\mathrm{w}} ; \frac{+}{+} ; \frac{+}{+}$

F2: $\quad \frac{\mathrm{w}}{\mathrm{Y}} ; \frac{\mathrm{P}\left[\mathrm{w}^{+} \text {Tip60-RNAi }\right]}{+} ; \frac{+}{+} \times \frac{\mathrm{w}}{\mathrm{w}} ; \frac{+}{+} ; \frac{+}{+}$

F3: $\quad \frac{\mathrm{w}}{\mathrm{Y}} ; \frac{\mathrm{P}\left[\mathrm{w}^{+} \text {Tip60-RNAi }\right]}{+} ; \frac{+}{+} \times \frac{\mathrm{w}}{\mathrm{w}} ; \frac{\mathrm{P}\left[\mathrm{w}^{+} \text {Tip60-RNAi }\right]}{+} ; \frac{+}{+}$

Briefly, males carrying the transposase $\Delta 2-3$ were crossed to females homozygous for the P-element with the sequence for dTip60-RNAi. In the F1 generation, both the transposase and the P-element are present, which leads to relocation of the P-element in the germline. Therefore, flies carrying the P-element on the autosome will be present in the F2 and are selected by the following crosses by taking advantage of the $\mathrm{X}$ chromosomal position of the white-gene and the previous location of the P-element. 


\subsection{Antibody generation}

A full-length dTip60 cDNA vector (LD31064) was obtained from Open Biosystems. Three dTip60 fragments were amplified from LD31064 and cloned into pET15b (Novagen). dTip60-) corresponded to amino acids 30-304, dTip60-B corresponded to amino acids 305-541, and dTip60 full corresponded to amino acids 30-541. The recombinant His-tagged fusion proteins dTip60-A and dTip60-B were purified using Talon beads (Clontech) according to the manufacturer's instruction. The respective eluate was further purified by seperating the proteins by SDS-PAGE, and the gel was stained with $1 \mathrm{M}$ ice-cold $\mathrm{KCl}$. The band representing the respective dTip60 fusion protein was cut out and the protein was electroeluted from the band. A small sample of purified dTip60-A and dTip60-B was assayed on SDS-PAGE and stained with Coomassie Blue (Figure 5). dTip60-A and dTip60-B were used for immunization of animals, and polyclonal dTip60-specific antibodies were raised in guinea pig (Pineda).
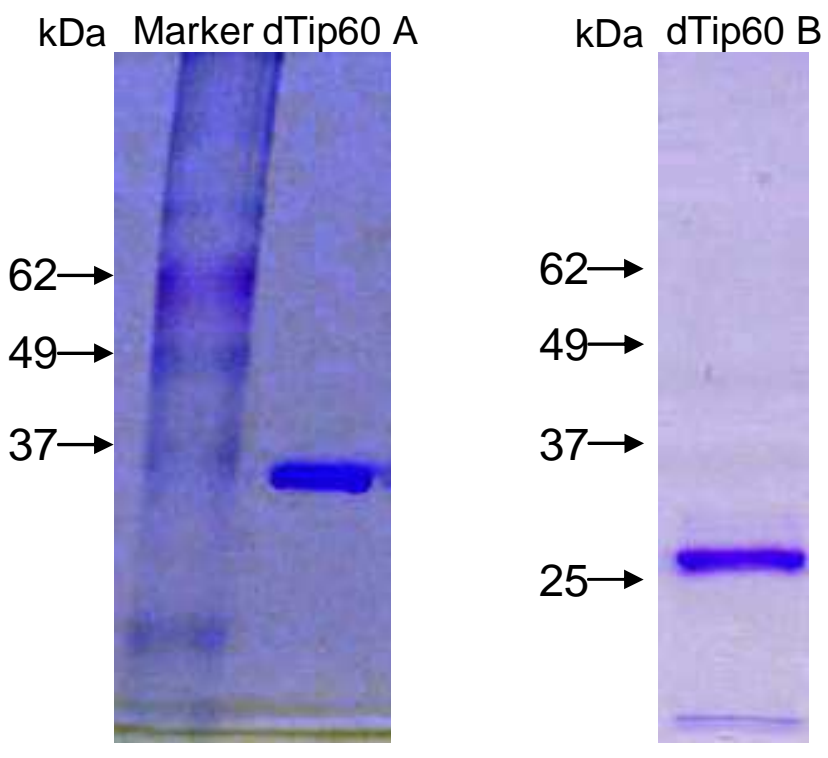

Figure 5: Samples of purified recombinant fusion proteins dTip60-A and dTip60-B as used for generation of a dTip60-specific antiserum. 


\subsection{Preparation of cell extracts, SDS-PAGE and Western blot}

For E. coli extracts, cells were induced with $1 \mathrm{mM}$ IPTG for three hours and collected by centrifugation. Cell pellets were resuspended in $100 \mu$ Lämmli buffer (Sambrook et al., 1989) and incubated for $10 \mathrm{~min}$ at $96^{\circ} \mathrm{C}$.

Western blot analysis on Kc nuclear extracts was performed as described previously (Eggert et al., 2004). Proteins were separated on 10\% SDS-polyacrylamide gels and blotted to nitrocellulose membranes, blocked and incubated with anti-dTip60 (1:5000) or preimmune serum (1:200) as primary and goat anti-guinea pig IgG (peroxidase conjugated, 1:5000, Sigma) as secondary antibody.

SDS-PAGE and Western blot analysis was performed according to standard procedures (Sambrook et al., 1989).

\subsection{Immunohistochemistry}

\subsubsection{Whole-mount fluorescent staining of Drosophila embryos}

Whole-mount fluorescent staining of Drosophila embryos was essentially performed as described previously (Kesper et al., 2007). Briefly, embryos were collected for 0-24 hours, washed in $0,7 \% \mathrm{NaCl}$ and dechorionated with agitation in $50 \%$ bleach/ $0,7 \%$ $\mathrm{NaCl}$ for $4 \mathrm{~min}$ in an eppendorf tube. After five washes with $0,7 \% \mathrm{NaCl}$, embryos were fixed for $30 \mathrm{~min}$ in $1 \mathrm{ml}$ fixing mixture $(500 \mu \mathrm{l}$ heptane, $450 \mu \mathrm{l} \mathrm{PBS}, 50 \mu 137 \%$ formaldehyde). The lower phase was removed and embryos were devitellinized by addition of $1 \mathrm{ml}$ of methanol and vigorous shaking by hand. Embryos were washed with ethanol and either stored at $-20^{\circ} \mathrm{C}$ or immediately used for experiments.

Embryos were rehydrated by washing with $70 \%$ ethanol, incubation in $50 \%$ ethanol for 5 minutes, and another $5 \mathrm{~min}$ in $30 \%$ ethanol. Next, embryos were washed with PBST (1 x PBS, 0,1\% Triton $\mathrm{X} 100)$ three times for $10 \mathrm{~min}$ at room temperature with mild agitation. After blocking for 30 minutes at room temperature with $5 \%$ heat inactivated goat serum (Dianova) in PBT, embryos were incubated with anti-dTip60 (1:400 in PBT) or preimmune serum $\left(1: 200\right.$, PBT) as a control overnight at $4{ }^{\circ} \mathrm{C}$ without agitation. After brief washing with PBT, embryos were incubated with goat anti-guinea pig Cy3 (1:200, Dianova) as a secondary antibody for 2 hours at room temperature. Embryos 
were washed four times for 10 min with PBT and mounted on slides.

Images were taken using a Zeiss Axiovert 200 microscope and electronically processed with the MetaMorph program (Molecular Devices).

\subsubsection{In situ staining of Drosophila embryos}

For in situ staining of Drosophila embryos, a digoxigenin-labeled RNA antisense probe specific for dTip60 mRNA was generated from a DNA template corresponding to base pairs 250- 813 of dTip60. This DNA template carried a T7 promotor on its 3' end. T7 transcription was carried out with DIG RNA labeling mix (Roche) according to manufacturer's instructions. As a control, a digoxigenin-labeled RNA sense probe was generated from a DNA template corresponding to base pairs 250 - 1116 of dTip60 with a 5' T7 promotor. After the synthesis of digoxigenin-labeled probes, the DNA template was digested with DNase I and the RNA probe was precipitated with $\mathrm{LiCl}$ and the pellet was resuspended in $\mathrm{H}_{2} \mathrm{O}$.

Embryos were washed, dechorionated, fixed and devitellinized as described (2.8.1). Rehydration was carried out in 50\% methanol in PBT and embryos were post-fixated for 10 min in 3,7\% formaledhyde in PBT. Next, embryos were washed five times in PBT for five min, in 50\% hybridisation buffer (50 ml formamid, $25 \mathrm{ml} 20 \mathrm{x} \mathrm{SSC}, 0.1$ $\mathrm{ml}$ 10\% Tween 20, ad $100 \mathrm{ml}$ aqua dest.) in PBT and for another $10 \mathrm{~min}$ in hybridisation buffer only. Prehybridisation was carried out in hybridisation buffer at $70^{\circ} \mathrm{C}$ for at least one hour. Digoxigenin-labeled RNA probe $(1: 1000$ in hybridisation buffer) was added and incubated at $70^{\circ} \mathrm{C}$ overnight. Embroys were washed once in hybridisation buffer for $30 \mathrm{~min}$, once in $50 \%$ hybridisation buffer/ PBT for $30 \mathrm{~min}$ and four times in PBT. Incubation with anti-digxigenin alkaline phosphatase coupled antibody (1: 2000, Roche) in PBT was carried out for one to four hours at room temperature. Embryos were washed three times for $20 \mathrm{~min}$ in PBT and three times for 5 min in freshly prepared reaction solution (100 mM Tris $\mathrm{pH}$ 9,5, $100 \mathrm{mM} \mathrm{NaCl}, 50 \mathrm{mM}$ $\mathrm{MgCl}_{2}$ ). Colour reaction was performed in BM purple AP substrate solution (Roche) until sufficient staining was visible. Colour reaction was stopped by washing with PBT and embryos were mounted on sildes. 
Table 6: Primers used for generation of in situ probes

\begin{tabular}{|l|l|l|}
\hline Name & Description & Sequence (5' to 3' direction) \\
\hline $250 \mathrm{fw}$ & $\begin{array}{l}\text { dTip60 antisense } \\
\text { probe for in situ } \\
\text { hybridisation }\end{array}$ & $\begin{array}{l}\text { CGC GGT ACC GCG GAG AGA CGG CTC ACA } \\
\text { AAC AGG }\end{array}$ \\
\cline { 3 - 4 } HFA rv & $\begin{array}{l}\text { TAA TAC GAC TCA CTA TAG GGG AAC TCT } \\
\text { GTC ATT ACG TAG AAG }\end{array}$ \\
\hline dsT fw & $\begin{array}{l}\text { dTip60 sense } \\
\text { probe for in situ } \\
\text { hybridisation } \\
\text { (control) }\end{array}$ & $\begin{array}{l}\text { TTA ATA CGA CTC ACT ATA GGG AGA AAC } \\
\text { AAG CGC TTG GAC GAG TGG }\end{array}$ \\
\cline { 3 - 3 } & $\begin{array}{l}\text { GCG CGC GAA TTC CCC TGG TAT TTC TCA } \\
\text { CCC TAT CCG }\end{array}$ \\
\hline
\end{tabular}

\subsubsection{Fluorescent staining of polytene chromosomes}

Polytene chromosomes were prepared from wandering third instar larvae and were essentially fixed and stained as described (Eggert et al., 2004). Briefly, polytene chromosomes were first fixed in $3.7 \%$ paraformaldehyde for $30 \mathrm{~s}$, then refixed in a solution of $50 \%$ glacial acetic acid and $3.7 \%$ formaldehyde for 4-5 min and squashed. Polytene chromosomes were incubated with anti-dTip60 (1:5000) and goat anti-guinea pig Cy3 (1:200).

\subsubsection{Staining of imaginal wing discs}

Wing imaginal discs were dissected from wandering third instar larvae and fixed with 4\% formaldehyde for immuno-labeling following standard procedures. Primary antibodies: guinea pig anti-dTip60 (1:400), mouse anti-engrailed/invected 4D9 (Developmental Studies Hybridoma Bank, University of Iowa), rabbit anti-trimethylHistone H3 (Lys27) (1:500, upstate). Secondary antibodies: goat anti-guinea pig Cy3, goat anti-mouse, goat-anti rabbit (all three 1:500, Dianova). Cell death was analyzed by acridine orange (Invitrogen, USA) vital staining as described (Kramer and Staveley, 2003). 


\section{9 dTip60 knock-down in SL2 cells}

Three double-stranded RNA (dsRNA) corresponding to different parts of dTip60 cDNA were synthesized: ds1 corresponded to base pairs 190 - 807, ds2 to base pairs 603 1116 and ds3 to base pairs 1321 - 1618. As a negative control, a dsRNA for eGFP was used, and as a positive control, RNAi knock-down was performed with a dsRNA against the kinase Aurora B (Muller et al., 2006). In a first step, a DNA template was synthesized by PCR using primers that added a T7 transcription site to both the 5' and 3 ' end. Transcription of these DNA templates by the T7 polymerase led to the formation of dsRNA. For this synthesis, the MEGAscript Kit (Ambion), which contains a T7 polymerase, was used according to the manufacturer's instruction. dsRNAs were precipitated with $\mathrm{LiCl}$, and RNA concentration was measured photometrically and visualised by agarose gelelektrophoresis.

$10^{6} \mathrm{SL} 2$ cells were incubated with $10 \mu \mathrm{g}$ dsRNA in $1 \mathrm{ml}$ serum-free medium with Pen/Strep $(50 \mathrm{U} / \mathrm{ml})$ with agitation at room temperature for $10 \mathrm{~min}$ and for $50 \mathrm{~min}$ at $26^{\circ} \mathrm{C}$ without agitation. $2 \mathrm{ml}$ medium with Pen/Strep and FCS to a final concentration of $10 \%$ were added. Cells were harvested at the indicated timepoints and RNA was extracted using the NucleoSpin RNA II kit (Macherey -Nagel).

\subsection{Genome-wide gene expression profiling}

Expression profiling was performed in two separate batches on three independent pairs of dTip60 RNAi- and eGFP-treated samples using Affymetrix GeneChip Drosophila 2.0 arrays. Two batches were assayed by ImaGenes (Berlin), one batch was assayed by Dr. Klein-Hitpaß (Institut für Zellbiologie, Universitätsklinikum Essen), who performed analysis of all three batches. RNA was amplified, labeled and hybridized according to manufacturer's instructions and scanned in a GeneChip 3000 scanner with G7 update. Array images (CEL files) were processed to determine signals and detection calls (Present, Absent, Marginal) for each probeset using the Affymetrix GCOS1.4 software (MAS 5.0 statistical algorithm). Scaling across all probesets to an average intensity of 1000 was used to compensate for variations in the amount and quality of the cRNA samples and other experimental variables of non-biological origin. Pairwise 
comparisons of treated versus control samples was carried out with GCOS1.4, which calculates the change as a Signal Log Ratio (basis 2) and the significance of each change in gene expression (change p-value) based on a Wilcoxon ranking test.

Probesets exhibiting a significant increase or decrease $(\mathrm{p} \leq 0.002)$ were identified by filtering using the Affymetrix Data Mining Tool 3.0. To limit the number of false positives, further target identification was restricted to those probesets that received at least one present detection call in the treated/control pair. The list of reliably measured probesets resulting from batch one (1 replicate each) was combined with the four target lists resulting of a cross-comparison of batch 2 arrays (2 replicates each). 1485 probesets representing 1390 genes, which exhibited increase or decrease change calls in $\geq 4$ of the 5 comparison were identified and defined as consistently regulated targets.

Microarray data have been deposited at NCBI Gene Expression Omnibus (http://www.ncbi.nlm.nih.gov/geo/).

\subsection{Quantitative real-time PCR}

cDNA was synthesized from total RNA using the SuperScript III kit (Invitrogen). Realtime PCR was performed using real master mix (5Prime) containing Sybr Green and analyzed in a Rotor Gene 3000 (Corbett). Samples were cycled 45 times for $15 \mathrm{sec}$ at $94^{\circ} \mathrm{C}, 30 \mathrm{sec}$ at $56^{\circ} \mathrm{C}$ and $40 \mathrm{sec}$ at $68^{\circ} \mathrm{C}$, and the $\mathrm{C}_{\mathrm{t}}$ value for each reaction was determined. For expression analysis, the difference in $C_{t}$ between treated and untreated samples was determined, with both samples normalized to actin expression. For ChIP analysis, a standard curve of input samples was used to calculate the amount of DNA precipitated relative to the input DNA. The primers used for quantitative PCR analysis of expression levels are given in Table 7. For semiquantitative PCR of dTip60 expression levels, the same primer pairs as for quantitative PCR were used. Primers specific for ORF were used for quantitative analysis of ChIP experiments and are given in Table 8. 
Table 7: Primers used for quantitative PCR (qPCR) in the body of the gene (5' to 3 ' direction).

\begin{tabular}{|l|l|l|}
\hline QPCR & Forward & Reverse \\
\hline Fz2 & CAGCAAAGAAAGAGCCCAAG & CCGACACATTGGTATGGTGA \\
\hline CG14273 & TGACGTTTGGCAAATTGAAA & GGAGCCAGGAGCACTATTTG \\
\hline CG8942 & CACGATGGCTATCAGCTCAA & ACCCATCCCATCGTATTCAA \\
\hline CG5397 & GAGCGAGGACGATGGTAGAG & GTGGGAGTCAGGACACTCGT \\
\hline Drip & GCCAGGATATCAAGGGATCA & AAGCAGGTGCGGACATTTAC \\
\hline Mth12 & CAGCATCCGCAAAGTGAAG & CGGGAATAAGTAATCCCTCGTAG \\
\hline CG10131 & ACATCCTGGAGAGCCAGTTG & GATGGCATAAAGGTGCTCGT \\
\hline Ac3 & CGTTCTGATAAACCGGCACT & TAGCCGCTAATTGGTTCGAG \\
\hline CG16888 & GATTGAGTTGCATCGGGAAC & TCAGCTTCATTCGATTCGTG \\
\hline Nvy & GCAGTACGTGACCAACAACG & ATACCCCGTATTCCCCGTAG \\
\hline CG11899 & CTGCAGTGGCTCTGAACTTG & GCGACAGAAAATTGGAGGAC \\
\hline Actin & CATCGTAAGGTGCTTCGTGA & GGAGGTTAGAGGGCGTGCTT \\
\hline & & \\
\hline
\end{tabular}

Table 8: Primers used for $\mathrm{qPCR}$ at open reading frames (ORFs)

\begin{tabular}{|l|l|l|}
\hline ORF & Forward & reverse \\
\hline $\begin{array}{l}\text { CG5397 } \\
\text { Prom }\end{array}$ & GGCCGCAAAAGTTTAGCTTAC & CCAAGATCACCACCGAAAGT \\
\hline Drip Prom & GAGTGCGCGCTAACTCAC & TGCTCGCAATTAACCTTCG \\
\hline $\begin{array}{l}\text { CG8942 } \\
\text { Prom }\end{array}$ & GTCGAGTCCTCAACGGATGT & CACCTGCGATCGGTTACAA \\
\hline background & CAATGATCCTGCATTGTAACCA & TGATGGCAATTGAAACCATC \\
\hline
\end{tabular}




\subsection{Chromatin Immunoprecipitation (ChIP)}

ChIP was performed according to standard protocols (Wu et al., 2003). Briefly, $10^{8}$ SL2 cells were crosslinked with $1 \%$ fomaldehyde for $10 \mathrm{~min}$ at room temperature. Cells were harvested by centrifugation and incubated for $10 \mathrm{~min}$ in SDS lysis buffer (1\% SDS, $10 \mathrm{mM}$ EDTA, $50 \mathrm{mM}$ Tris-HCl, $\mathrm{pH} 8,1)$ with intermittent agitation. The cell lysate was sonicated for 30 cycles ( $10 \mathrm{sec}$. on, $30 \mathrm{sec}$. off) and the average length of the resulting DNA fragments was assayed to be approximately 500 bp by agarose gelelectrophoresis. After centrifugation, lysate was diluted tenfold in IP buffer $(0,01 \%$ SDS; 1,1\% Triton X-100; 1,2 mM EDTA; 16,7 mM Tris-HCl, pH 8,1; 16,7 mM NaCl; $1 \mathrm{mM}$ PMSF; Roche complete proteinase inhibitor).

ChIP was performed using $6 \mu 1$ dTip60 antiserum or preimmune serum on $300 \mu 1$ precleared chromatin solution with $35 \mu 1$ of $50 \%$ prewashed protein A sepharose beads at $4^{\circ} \mathrm{C}$ for two hours. $150 \mu \mathrm{l}$ cell lysate was set aside as input. Protein A sepharose beads were collected by brief centrifugation and washed five times with low or high salt buffer (0,1\% SDS, $1 \%$ Triton X-100, 2 mM EDTA, 20 mM Tris-HCl, pH 8,1, low 150 $\mathrm{mM} \mathrm{NaCl}$, high $500 \mathrm{mM} \mathrm{NaCl})$, and finally with lithium wash buffer $(0,25 \mathrm{M} \mathrm{LiCl}, 1 \%$ NP-40, $1 \%$ sodium deoxycholate, $1 \mathrm{mM}$ EDTA, $10 \mathrm{mM}$ Tris- $\mathrm{HCl} \mathrm{pH}$ 8,1). ProteinDNA complexes were eluted by adding $250 \mu \mathrm{l}$ of elution buffer (1\% SDS, 0,1M $\mathrm{NaHCO}_{3}$ ) for $15 \mathrm{~min}$ at room temperature. To reverse formaldehyde crosslinks, $20 \mu 15$ $\mathrm{M} \mathrm{NaCl}$ was added to the eluates and input, and incubated at $65^{\circ} \mathrm{C}$ for 4 hours. Next, 10 $\mu 1$ of $0,5 \mathrm{M}$ EDTA, $20 \mu \mathrm{l}$ of $1 \mathrm{M}$ Tris- $\mathrm{Cl}(\mathrm{pH} 6,5)$, and $2 \mu 1$ of $10 \mathrm{mg} / \mathrm{ml}$ proteinase $\mathrm{K}$ were added and incubated at $45{ }^{\circ} \mathrm{C}$ for 1 hour. Phenol/chloroform extraction was performed and DNA was precipitated with ethanol. DNA pellets were dissolved in $30 \mu 1$ of ultrapure $\mathrm{H}_{2} \mathrm{O}$ for further experiments.

\subsection{Bioinformatics}

For GOstat analyses of differentially regulated gene products, the software at http://gostat.wehi.edu.au (Beissbarth and Speed, 2004) was used.

Two batches of gene expression data were compared with the help of bioinformatics: one group contained genes up- or downregulated upon dTip60 loss as determined in this work. The other group contained gene expression signatures of HDAC1 or HDAC3 
RNAi and TSA treatment as determined by (Foglietti et al., 2006). We asked if there is an overlap of respective subgroups based on flybase numbers. We evaluated whether this overlap is significant, with 13966 currently defined as the totality of Drosophila genes by Flybase. P-values were determined using Fisher's exact test as implemented in the statistical software R, version 2.7.1. 


\section{Results}

\subsection{Phenotypes of dTip60 knock-down in Drosophila}

\subsubsection{Use of the Gal4/UAS system for RNA interference in flies}

The observation that dTip60 is a histone acetyltransferase suggests a function in gene regulation. In order to investigate dTip60 function in the organismal context, we sought to characterize the consequences of the loss of dTip60 in flies with the help of the Gal4/UAS system (Brand and Perrimon, 1993). This system makes use of P-elements, which are class II transponsons specific for Drosophila and allow the random insertion of genetic information in the genome. Flies are transformed with a P-element that contains the Gal4 Upstream Activating Sequence (UAS), a genetic marker (e.g. a gene that influences the eye colour) and the desired genetic element. This element is transcribed when the Gal4 protein is present, which binds to the UAS and activates transcription. Gal4 is a yeast-specific protein and is expressed as a transgene in a variety of Drosophila fly lines under the control of diverse promotors that are active in a defined tissue or at a defined point of time during development. The GAL4 transcriptional activator protein has a relatively high basal activity, but modulation of this activity as a function of temperature is possible (McGuire et al., 2004). In short, crossing a UAS line to a Gal4 line leads to expression of the genetic information of interest in the F1 generation, with the temporal and spatial expression depending on the properties of the Gal4 line chosen.

The Gal4/UAS system can be used for RNAi knock down of a gene of interest by constructing an inverted repeat containing part of the DNA of the gene of interest. Transcription of this inverted repeat leads to formation of a double-strand RNA (dsRNA), which is then degraded by the RNAi machinery and leads to downregulation of the respective gene (Figure 6). 


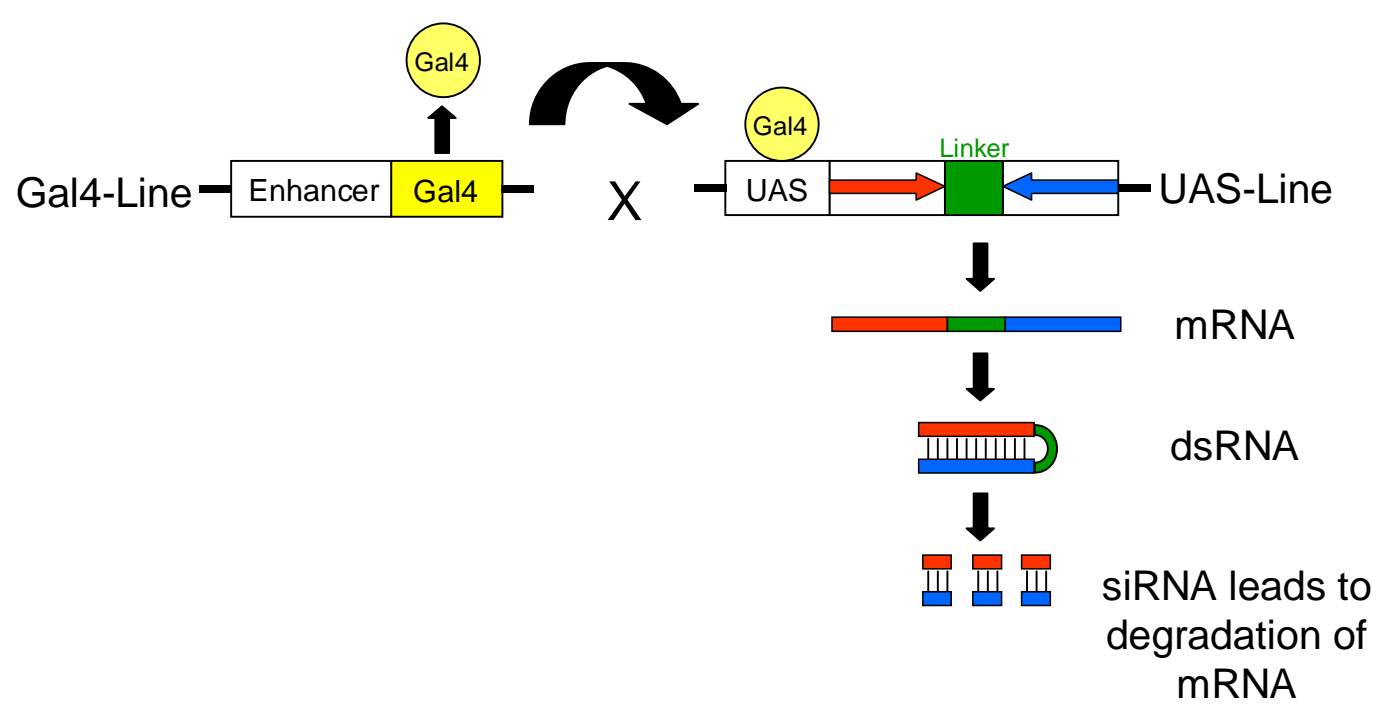

Figure 6: Mechanism of RNAi knock-down induced by the Gal4/UAS system.

\subsubsection{Strong knock-down of dTip60 led to lethality, whereas moderate knock-down caused developmental defects in the wing}

In order to characterize dTip60 loss in flies, we generated a dTip60-RNAi line using the Gal4/UAS system. Flies were transformed with a Gal4-driven dTip60 RNAi construct, and one transgenic line was obtained. The position of the sense- and antisense fragments of this construct relative to the dTip60 cDNA are given in Figure 7. The transgenic line, which carried the P-element insertion on the X-chromosome, was further used to obtain eight autosomal P-element insertions through mobilization of this P-element by crossing it to a line expressing transposase. All lines showed the same phenotypes described here, indicating that the effects of dTip60 knock-down were independent of the chromosomal localization of the P-element. Flies with the Gal4dTip60 RNAi P-element were homozygously or hemizygously viable, fertile and did not show signs of abnormal development. 

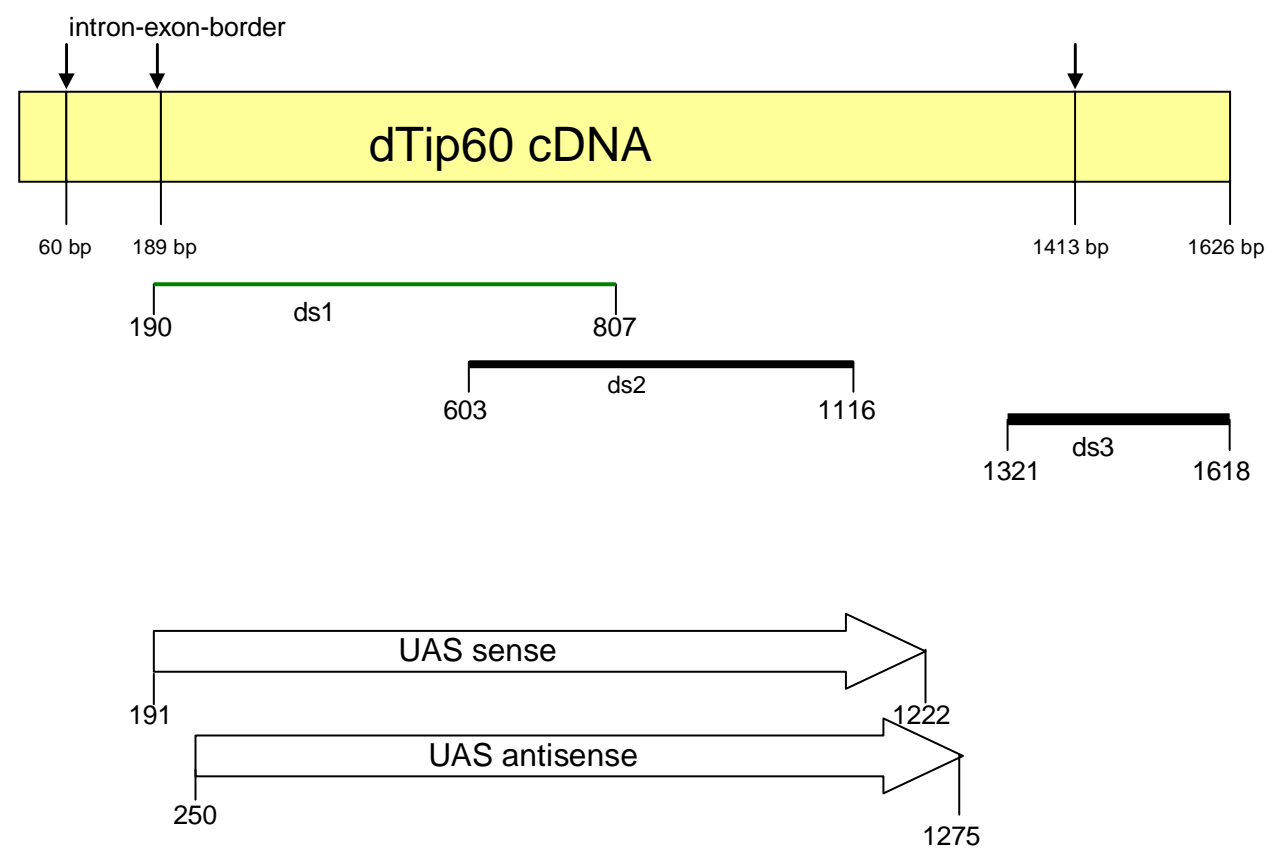

Figure 7: Overview of dsRNA constructs used for construction of the UAS-Gal4 RNAi fly line (UAS sense and antisense) and RNAi in SL2 cells (ds1, ds2, ds3). Arrows indicate intron-exon-borders of dTip60 cDNA.

In order to induce dTip60 RNAi expression, we crossed the insertion line to different Gal4 driver lines. Knock-down of dTip60 under the control of the daughterless promoter (da-Gal4), which is an early and ubiquitous driver line (Gortchakov et al., 2005), caused pupal lethality at $29^{\circ} \mathrm{C}$. This effect was reproduciable with the T80-Gal4 driver line at $29^{\circ} \mathrm{C}$ (Table 1), which is a late and ubiquitous driver line (Negeri et al., 2002). This indicated that dTip60 is essential, which was consistent with previous work (Zhu et al., 2007). 
Table 9. Phenotypes of dTip60 RNAi knock-down in flies

\begin{tabular}{|c|c|c|}
\hline Cross (F0) & Gal4 expression line & Phenotype (F1) \\
\hline $\begin{array}{l}\text { da-Gal4 x } \\
\text { dTip60-RNAi }\end{array}$ & $\begin{array}{l}\text { da }=\text { daughterless promotor } \\
\text { (early and ubiquitous } \\
\text { expression) }\end{array}$ & $\begin{array}{ll}29^{\circ} \mathrm{C}: & 100 \% \text { lethal } \\
25^{\circ} \mathrm{C}: & \text { few escapers } \\
22^{\circ} \mathrm{C}: & \text { no effect }\end{array}$ \\
\hline $\begin{array}{l}\text { T80-Gal4 x } \\
\text { dTip60-RNAi }\end{array}$ & $\begin{array}{l}\text { T80 promotor } \\
\text { (late and ubiquitous } \\
\text { expression) }\end{array}$ & $\begin{aligned} 29^{\circ} \mathrm{C}: & >90 \% \text { lethality } \\
27^{\circ} \mathrm{C}: & \text { little lethality } \\
& \text { survivors } \sim 100 \% \text { ectopic wing veins } \\
25^{\circ} \mathrm{C}: & \text { no lethality } \\
& \text { ca. } 50 \% \text { ectopic wing veins }\end{aligned}$ \\
\hline $\begin{array}{l}\text { en-Gal4 x } \\
\text { dTip60-RNAi }\end{array}$ & $\begin{array}{l}\text { en = engrailed promotor } \\
\text { (early expression in part of } \\
\text { the posterior imaginal wing } \\
\text { disc) }\end{array}$ & $\begin{aligned} 29^{\circ} \mathrm{C}: & \text { no lethality } \\
& \text { ca. } 50 \% \text { ectopic wing veins }\end{aligned}$ \\
\hline
\end{tabular}

At $27^{\circ} \mathrm{C}$, only little lethality was observed in the T80 cross, and more than $90 \%$ of the survivors showed ectopic veins parallel to L5 (Figure 8). These ectopic veins varied in length, with most of them of medium length. They often occurred at a similar position close to the posterior crossvein. This effect was also observed, although at lower frequency, using a Gal4-driver line under the control of the engrailed promotor (enGal4) at $29^{\circ} \mathrm{C}$, which is expressed early in the posterior part of the wing imaginal disc. At low frequency, a bulge close to L3 was observed in both en- and T80-driven dTip60RNAi. Occasionally, the formation of ectopic veins in other regions of the wing was observed parallel to L1, L2 and L3 (Figure 9). A further decrease of temperature led to reduced lethality in da-Gal4 driven dTip60-RNAi flies and reduced the formation of ectopic veins in T80-Gal4 driven dTip60-RNAi flies. 

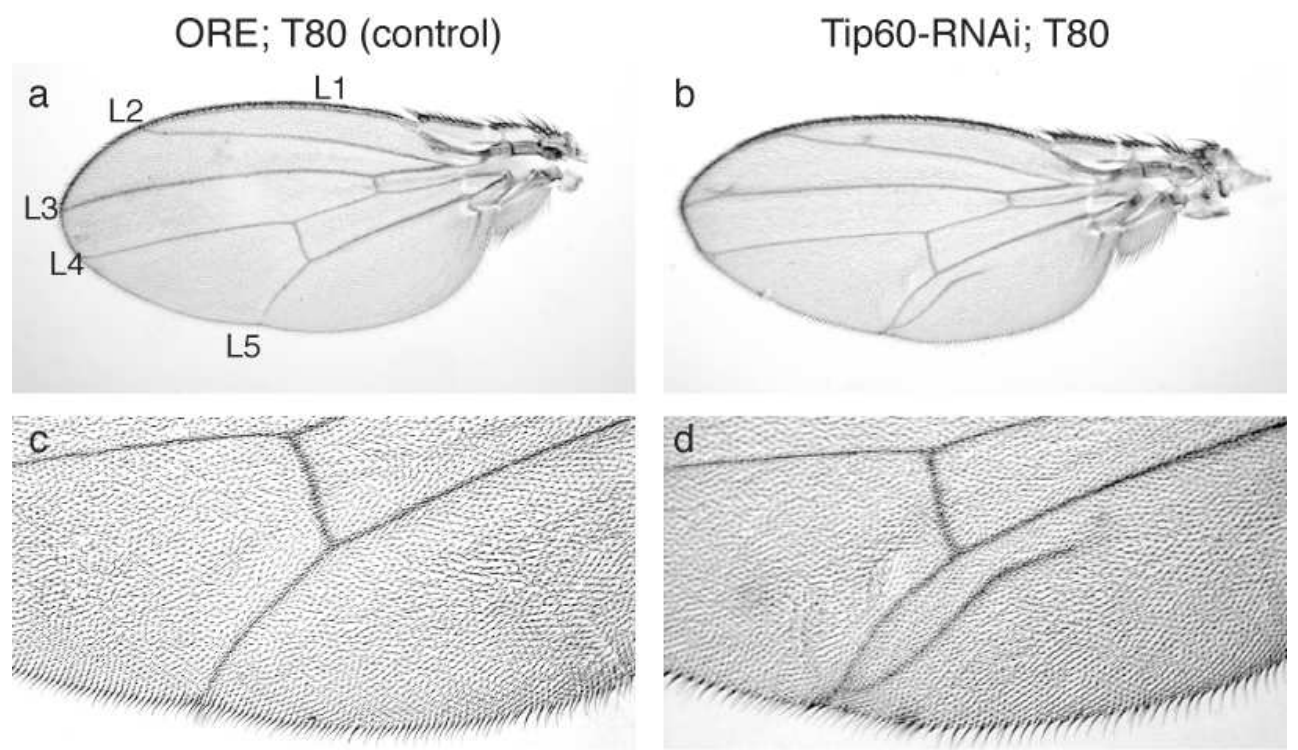

Figure 8: Downregulation of dTip60 by RNAi resulted in defects in the Drosophila wing. a) Wild-type wing with longitudinal veins L1 - L5 indicated and b) wing from a representative fly with dTip60-RNAi driven by T80-Gal4. Magnification of the area around L5 in wild-type (c) and dTip60-RNAi (d).

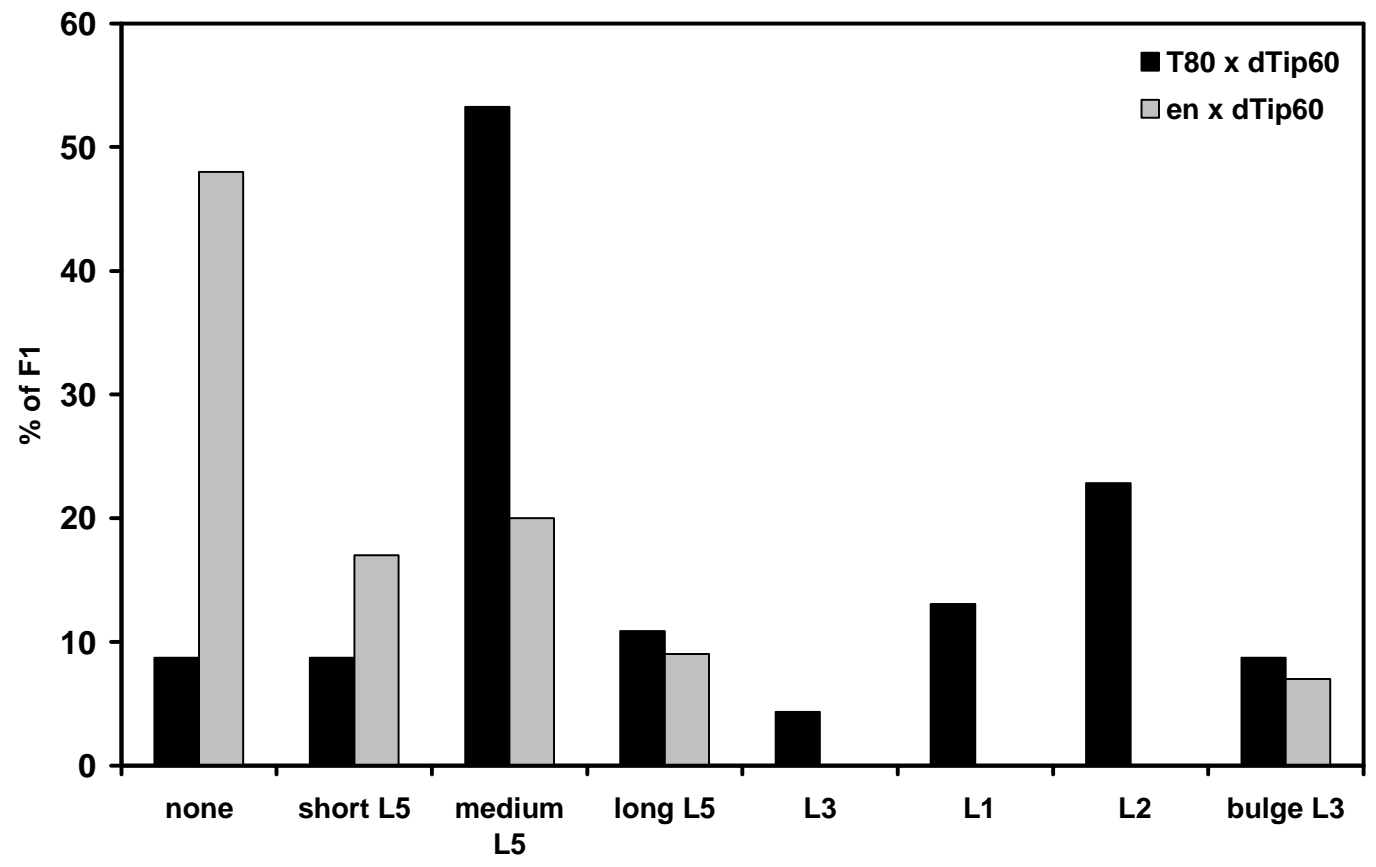

Figure 9: Position, length and frequency of ectopic wing veins caused by dTip60-RNAi driven by T80 or en. 


\subsubsection{Knock-down of dTip60 in the imaginal wing disc led to apoptosis and enrichment of H3K27me3}

We further evaluated the effect of dTip60-RNAi in the wing imaginal disc. In order to enhance the wing phenotype, we combined dTip60 knock-down with Gal4-driven expression of Dicer, a component of the RNAi machinery (Hammond et al., 2000). Here, en-Gal4 led to a Notch phenotype (data not shown), which was consistent with dTip60 playing a role in role in Notch signaling (Gause et al., 2006).

In the en-expressing part of the disc, dTip60 levels were weakly but visibly reduced (Figure 10). This caused increased apoptosis as measured by staining with acridine orange. Furthermore, the level of H3 K27 tri-methylation was increased (Figure 10). Since this is a chromatin mark associated with PRC2-dependent gene repression (Czermin et al., 2002; Muller et al., 2002), this suggested that dTip60 loss resulted in a global loss of gene expression.
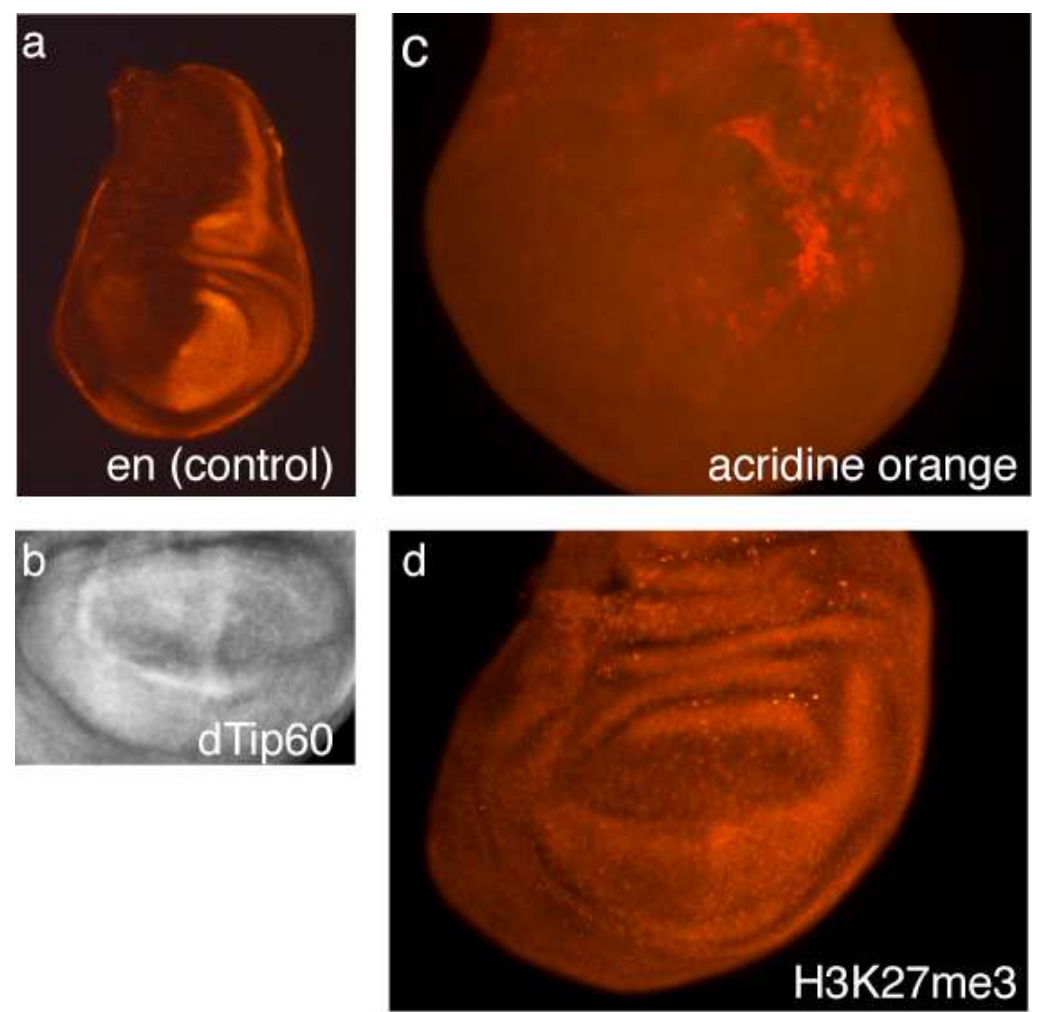

Figure 10: Effects of en-Gal4-driven dTip60 downregulation in the imaginal wing disc. a) en expression in the posterior part of the imaginal wing disc. b) en-Gal4-driven dTip60-RNAi caused reduced levels of dTip60 in the posterior part of the imaginal wing disc. c) Reduction of dTip60 protein level led to increased cell death as visualized by acridine orange staining. d) H3K27me3 increased upon decrease of dTip60 protein levels. (Images courtesy of Prof. Ansgar Klebes.) 


\subsection{Generation of an antiserum specific for dTip60}

In order to further characterize the properties of dTip60, an antibody was raised against

a fragment spanning the N-terminal amino acids 30 - 304 of dTip60 (dTip60-A).

Western blotting of E. coli extracts containing recombinant dTip60 showed that the antiserum gave a strong band for the $\mathrm{N}$-terminal dTip60 fragment which was used for immunization (dTip60-A), but not for a C-terminal dTip60 fragment (dTip60-B; spanning amino acids 305-541). The antiserum also gave a clear band for full length recominant dTip60 (Figure 11 A).

Nuclear cell extracts from cultured Drosophila cells showed a single band for dTip60, while the preimmunse serum from the animal in which the antibody was raised gave only weak background signals (Figure 11 B). This suggested that the antibody was specific to dTip60.

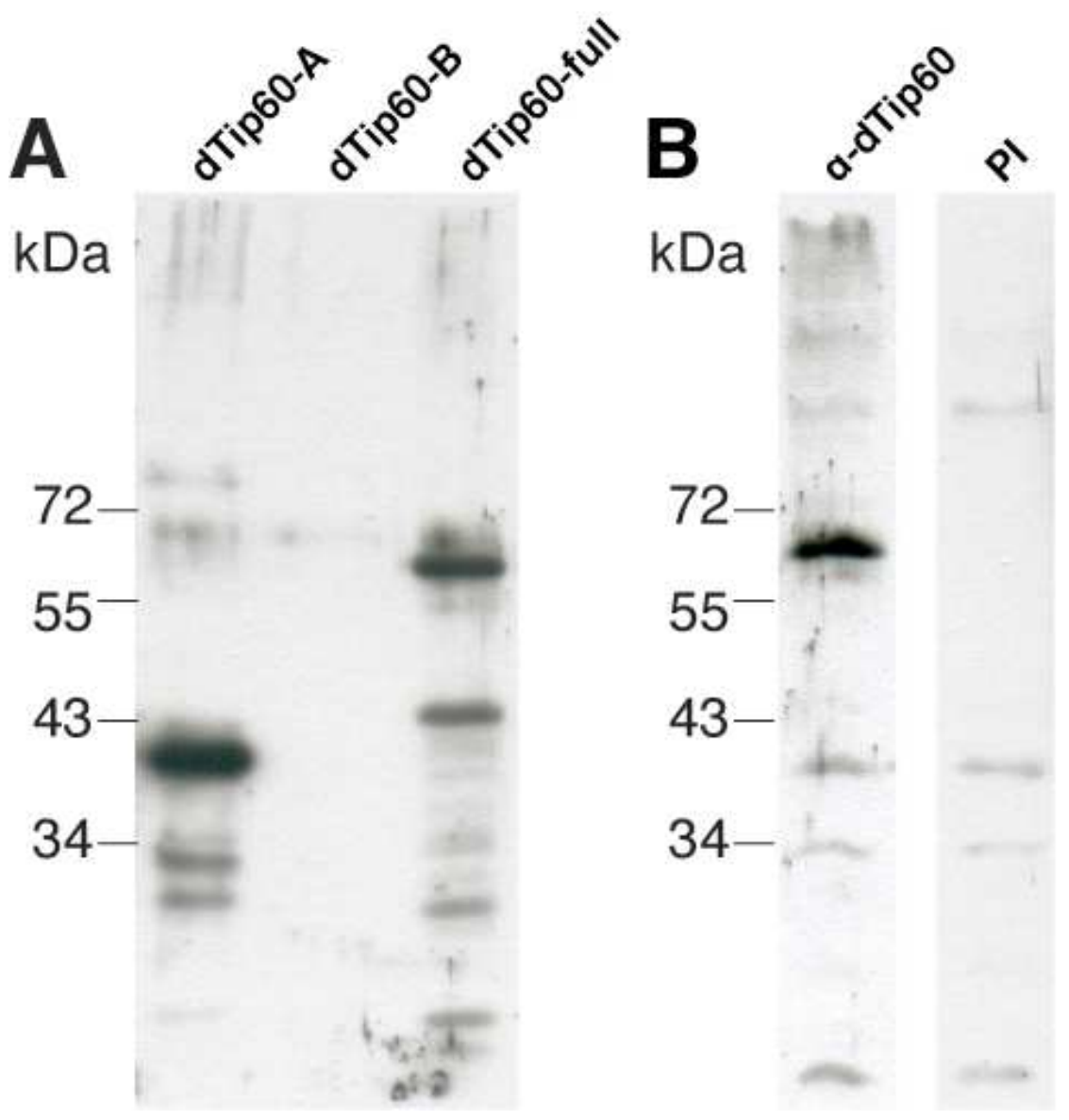

Figure 11: Western blot analysis indicated that the antiserum raised against dTip60 recognized dTip60 protein. (A) Whole cell extracts from E. coli expressing recominant His-tagged dTip60. (B) Nuclear extracts from cultured Drosophila cells probed with anti-dTip60 serum or preimmune serum from the aninmal in which the antiserum was raised. 


\subsection{Nuclear localization of dTip60 during embryonic development}

We were interested in evaluating the localization of dTip60 during embryonic development. The histone acetyltransferase function of dTip60 as well as its potential function in gene regulation might suggest a nuclear localization. In order to determine when and where dTip60 is present during embryonic development, we performed whole-mount fluorescent dTip60 antibody staining of Drosophila embryos. Significantly, dTip60 showed nuclear localization in embryos starting at stage 3 (Figure 12A). A weak staining of the yolk was also observed.

In the nucleus of blastoderm cells in the early Drosophila embryo, a heterochromatin region facing outward of the embryo can be visualized by microscopy. We asked how localization of dTip60 related to this heterochromatic region and performed staining of late-stage embryos with DAPI and for dTip60. The heterochromatic region within the nucleus gave a bright DAPI staining (Figure 6B (a) and Figure 6C (a)). dTip60 also localized to the nuclei of blastoderm embryos. It was predominantly enriched in euchromatic regions and showed lesser staining of the peripheral heterochomatic chromosomal areas (Figure 6B, C).

Next, we wanted to gain insight into the distribution of dTip60 mRNA in Drosophila embryos. To this end, we performed in situ staining of embryos with a dTip60-specific probe. We found that at age $20-24$ hours, embryos showed ubiquitous staining for dTip60 (Figure 13, p. 45).

Altogether, these findings indicated a nuclear localization of dTip60 beginning at early stages of embryonic development. 

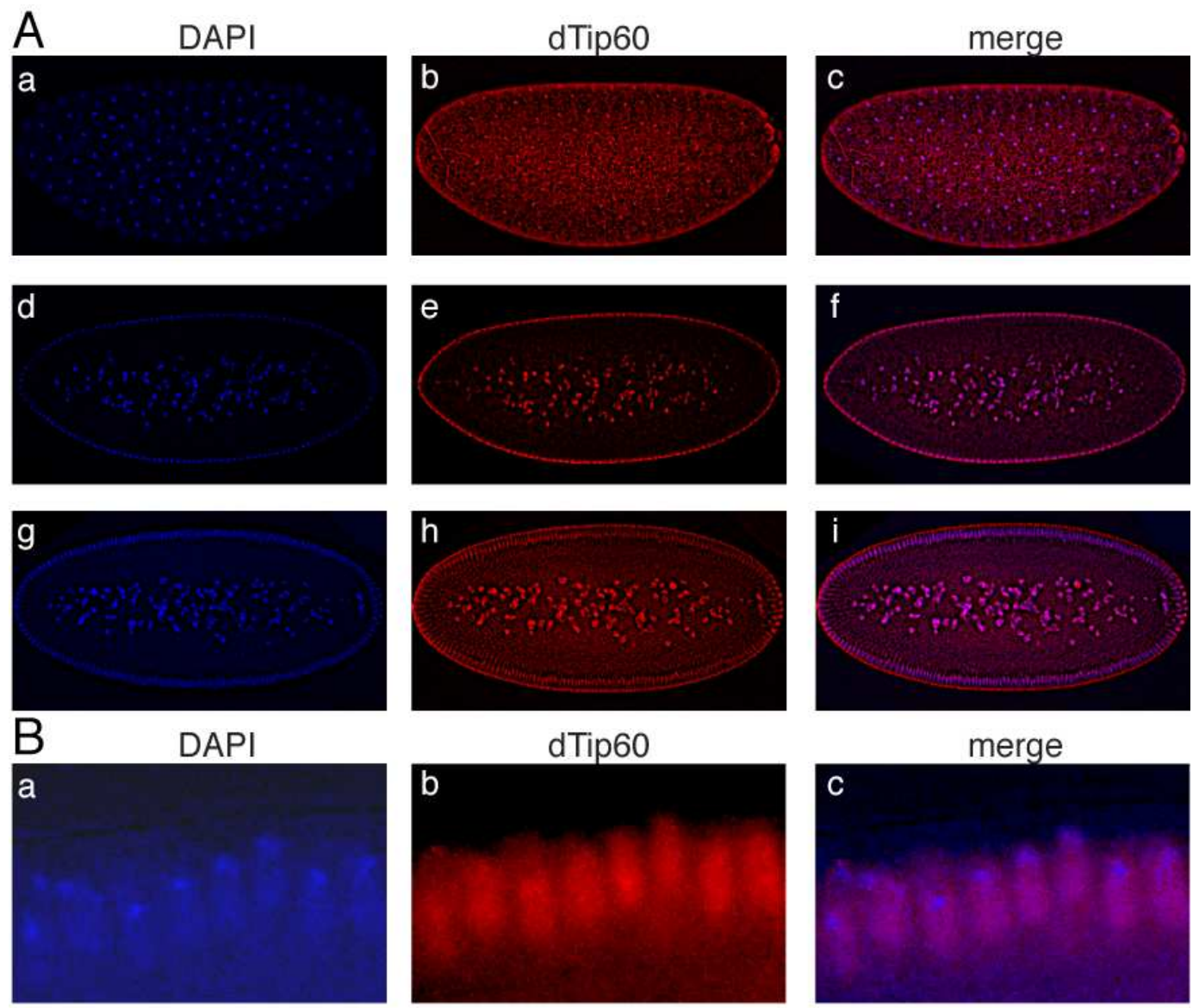

dTip60

merge
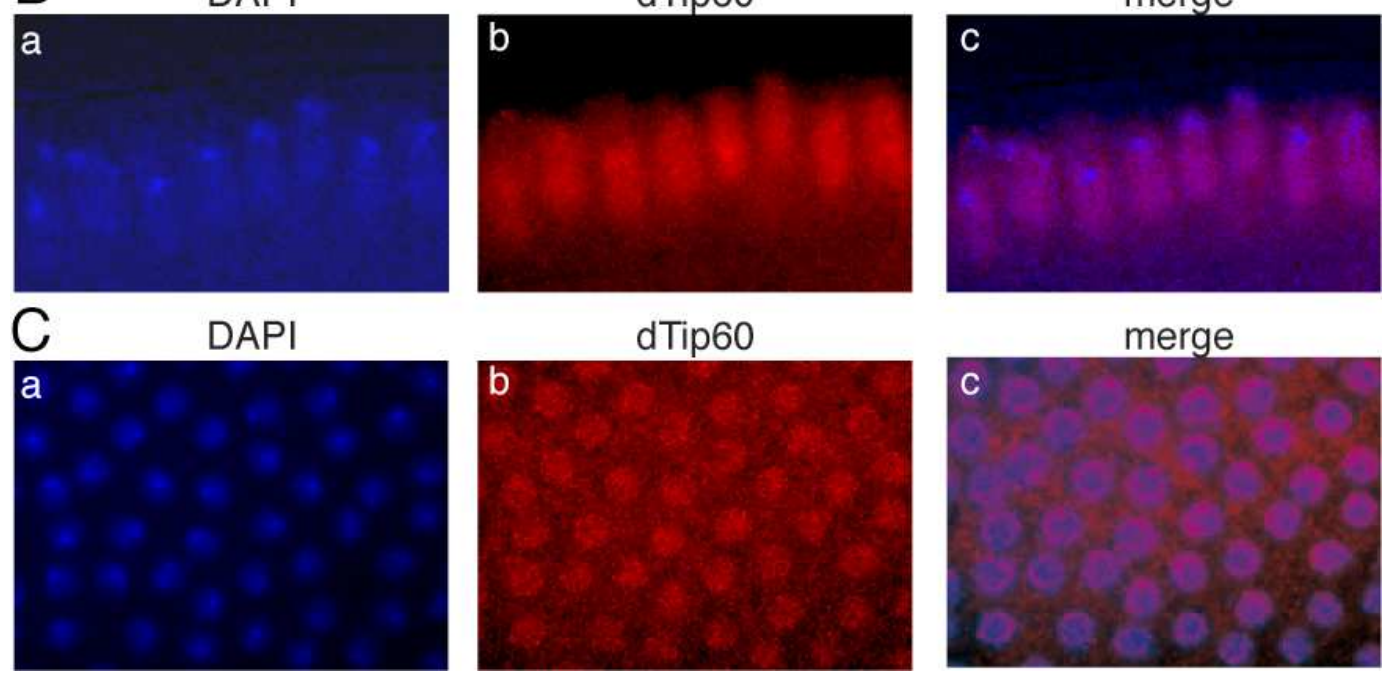

dTip60

merge
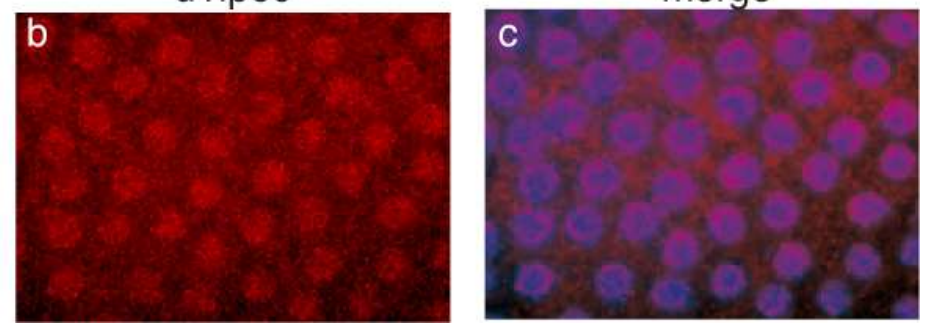

Figure 12: Localization of the dTip60 protein in Drosophila embryos. (A) Early embryos from wild-type flies were double labelled with DAPI (blue, a, d, g) and the dTip60 antibody (red, b, e, h). dTip60 was present in embryos after stage 3 (a-c) and mainly stained the nuclei of yolk cells and the blastoderm (d-i). (B) and (C) Nuclear localization of dTip60 in the blastoderm embryo. dTip60 was enriched in the euchromatic region of chromosomes (with technical help from Dörthe Kesper). 

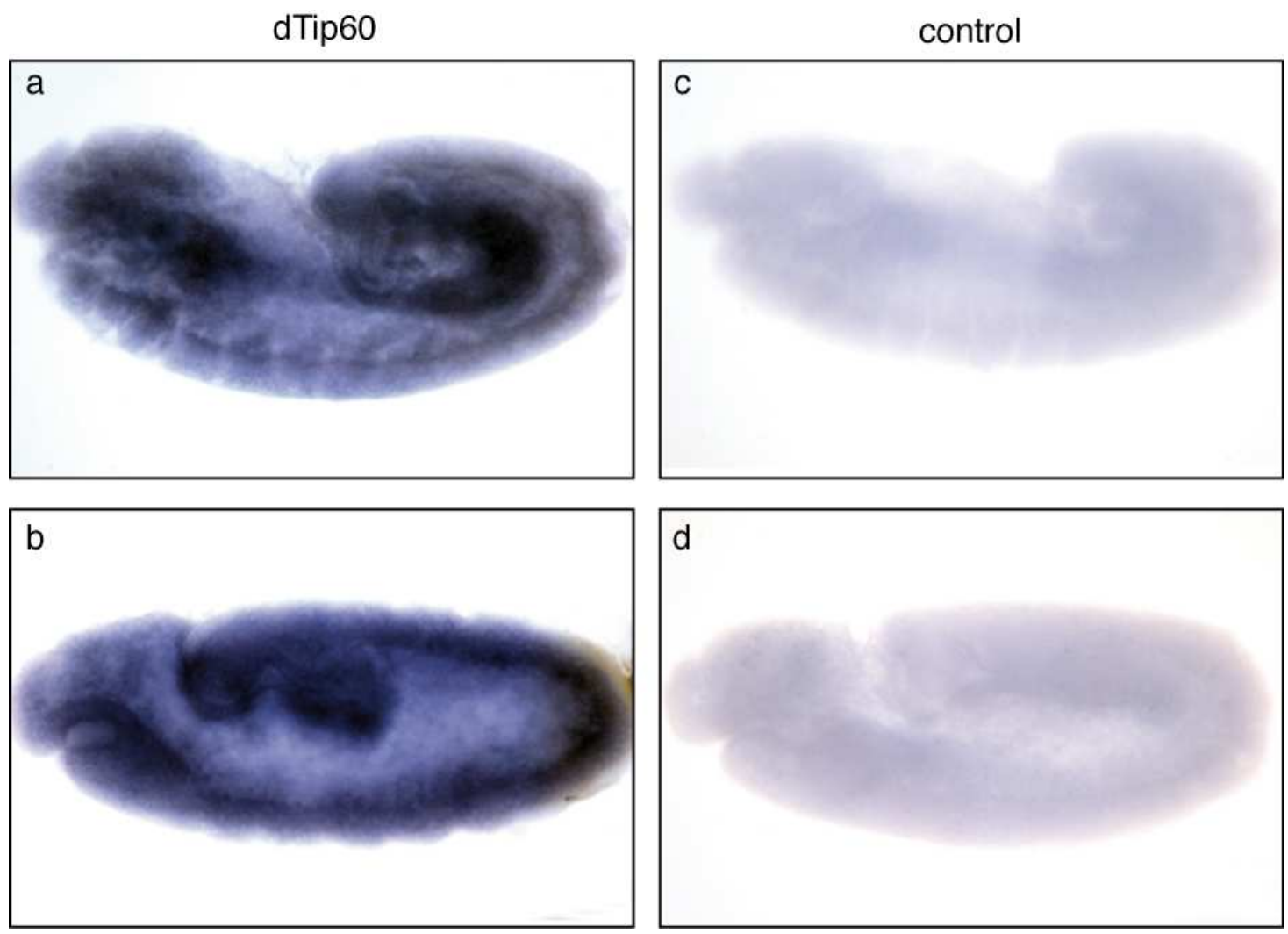

Figure 13: Ubiquitous expression of dTip60 RNA during embryogenesis. Lateral views of embryos hybridized with digoxigenin-labeled RNA probes of dTip60 in antisense $(a, b)$ and, as a control, in sense (c, d) orientation (with technical help from Dörthe Kesper).

\section{4 dTip60 localized to interbands on polytene chromosomes}

In order to gain further insight into both the chromosomal localization of dTip60 and its possible role in gene regulation, we made use of the polytene chromosomes in the salivary glands of Drosophila L3 larvae. Polytene chromosomes occur when cells of a specialized tissue, i. e. salivary gland cells, undergo several rounds of replication, but no cell divisions (endomitosis). These multiple rounds of replication lead to the formation of polytene chromosomes, where homologous chromatids remain together. Polytene chromosomes are organized in bands and interbands, which are the result of differential chromatin compaction. Inactive genes are frequently located in the heterochromatic bands, whereas actively transcribed genes are thought to be located in the euchromatic interbands (Ebert et al., 2006).

We asked where dTip60 is localized on polytene chromosomes. Staining of dTip60 on polytene chromosomes showed that it localized to a subset of interbands (Figure 14), where it was restricted to defined regions within the interbands. The overlay of the 
DNA staining with the dTip60 staining showed that the dTip60 staining intercalated with the DNA signal, which stains the highly compacted DNA of the bands. Overall, dTip60 was present in roughly $50 \%$ of the interbands, where it was either localized in compact dots within the interband, or in a narrow stripe next to a band. Comparison of the dTip60 pattern with patterns of other proteins known to be located at interband regions showed that the dTip60 pattern was distinct from the patterns of BEAF and Modmdg4 (data not shown).

In summary, dTip60 was localized to interbands, often staining the region adjacent to a band or producing a punctate pattern within a region of the interband. The localization of dTip60 at interbands was consistent with a role for dTip60 in gene regulation.
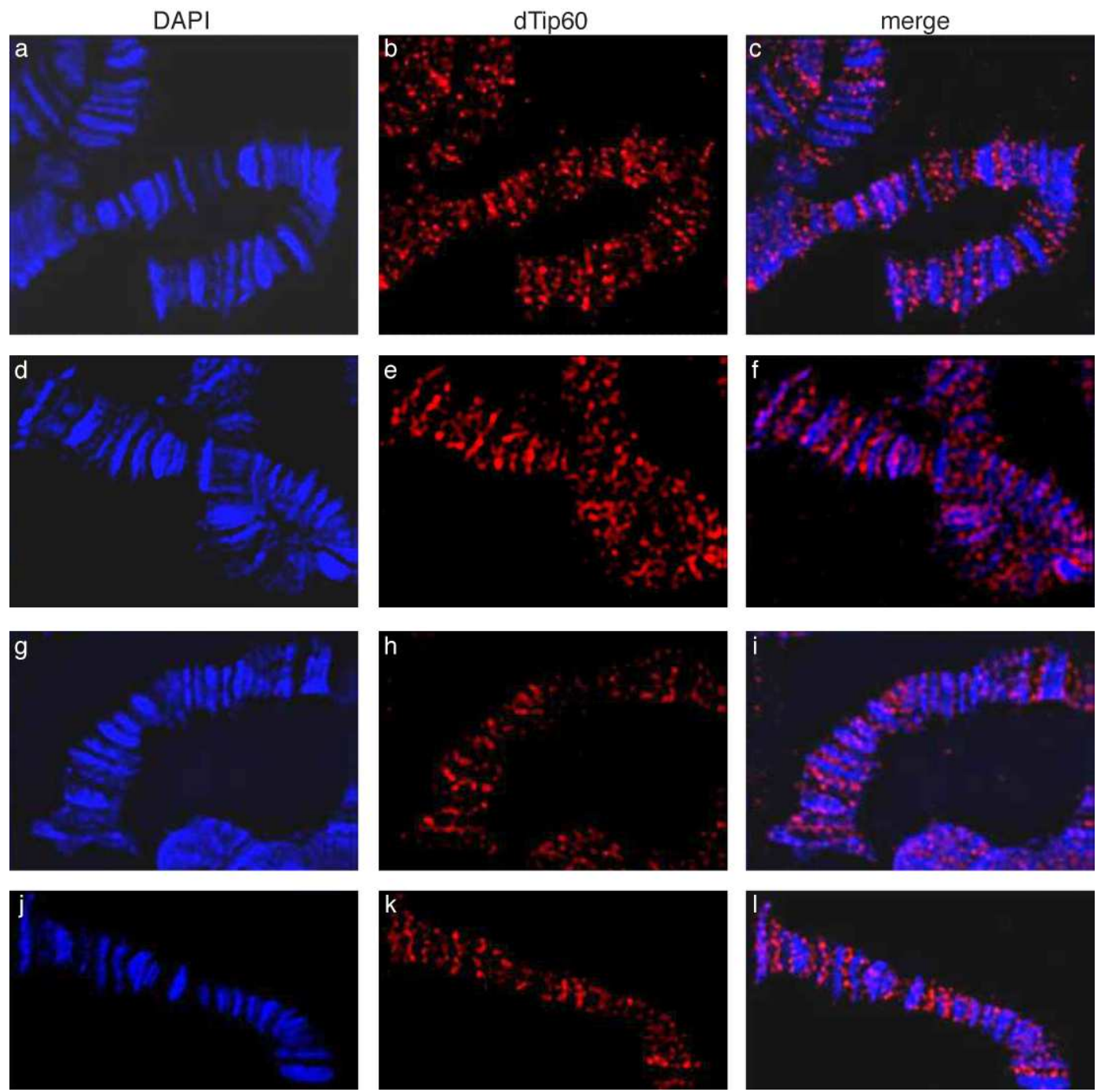

Figure 14: dTip60 localized to interbands of polytene chromosomes. Four different views of chromosome 3L showing the distal area with region 61 stained for DNA with DAPI (blue, a, d, g, j) and for dTip60 (red, b, e, h, k). Image: Prof. Harald Saumweber. 
Next, we asked whether dTip60 staining was reduced on polytene chromosomes from dTip60-RNAi larvae. dTip60-RNAi was driven by da-Gal4, whereas a cross of ORE to da-Gal4 was used as a control. The experiment was carried out at $29^{\circ} \mathrm{C}$ to enhance the RNAi knock down.

In dTip60-RNAi animals, dTip60 signal was strongly reduced in comparison to the control. The remaining background staining did not show a distinct banding pattern. The banding pattern of dTip60 appeared clearly in animals where dTip60 was present, whereas in dTip60-RNAi animals, only little signal remained at all. These findings show that the level of dTip60 protein was reduced on polytene chromosomes of dTip60RNAi animals.

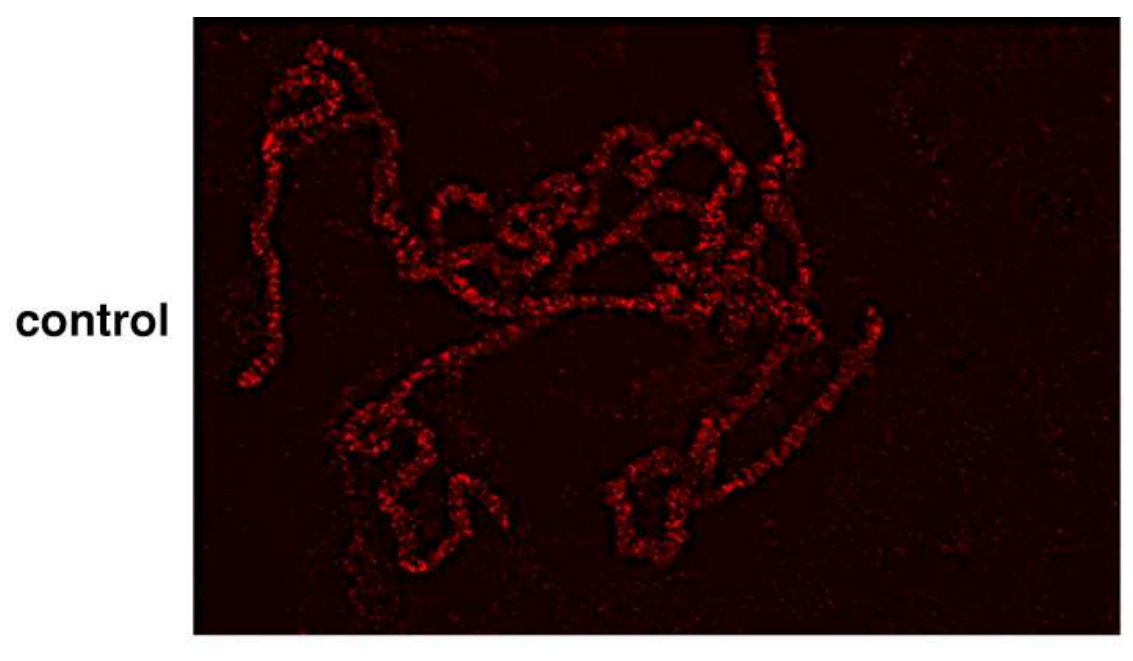

\section{dTip60-RNAi}

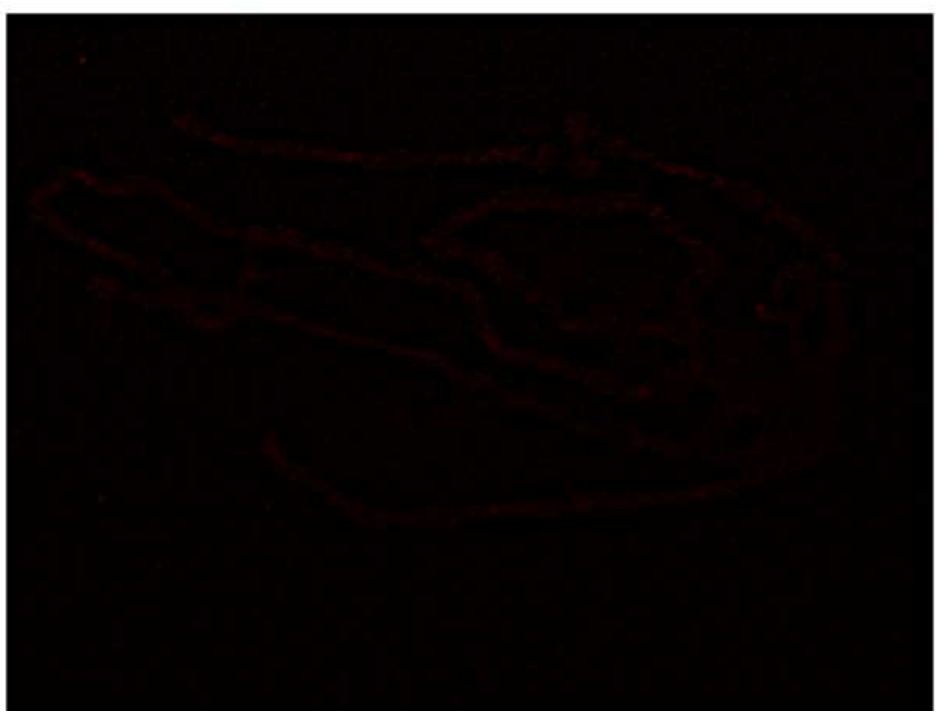

Figure 15: dTip60 is reduced on polytene chromosomes of dTip60-RNAi animals. Comparison of control (top) and dTip60-RNAi (bottom) reveal that dTip60 staining reduced in dTip60-RNAi animals. Images were electronically processed (right panel) in order to reduce optical distortion. 


\subsection{Genome-wide expression analysis revealed a major role for dTip60 in gene regulation}

Next, we sought to identify genes that are regulated by dTip60. A priori, one might expect dTip60 to activate transcription of target genes because of its function as a histone acetyltransferase. To test this, we used RNAi to downregulate dTip60 in SL2 cells and compared the genome-wide expression profiles to those of mock-treated cells. An overview of the position and lenght of constructs used for RNAi experiments is given in Figure 7.

We performed RNAi of dTip60 in SL2 cells using the dsRNAs ds1, ds2 and ds3. Mocktreatment with RNAi against eGFP, which is not present in Drosophila cells, was used as a negative control. RNAi against Aurora B (Aur), an essential kinase whose loss leads to a visible cell phenotype (Lange et al., 2002) was used as a positive control. Cells were counted after 2 and 5 days of RNAi-treatment. Proliferation was reduced in comparison to mock-treated cells upon treatment with the three dsRNAs directed against dTip60, as well as with the dsRNA against Aur (Figure 16). The reduced cell proliferation upon dTip60-RNAi reflected the fact that dTip60 was essential in SL2 cells (Kusch et al., 2004).

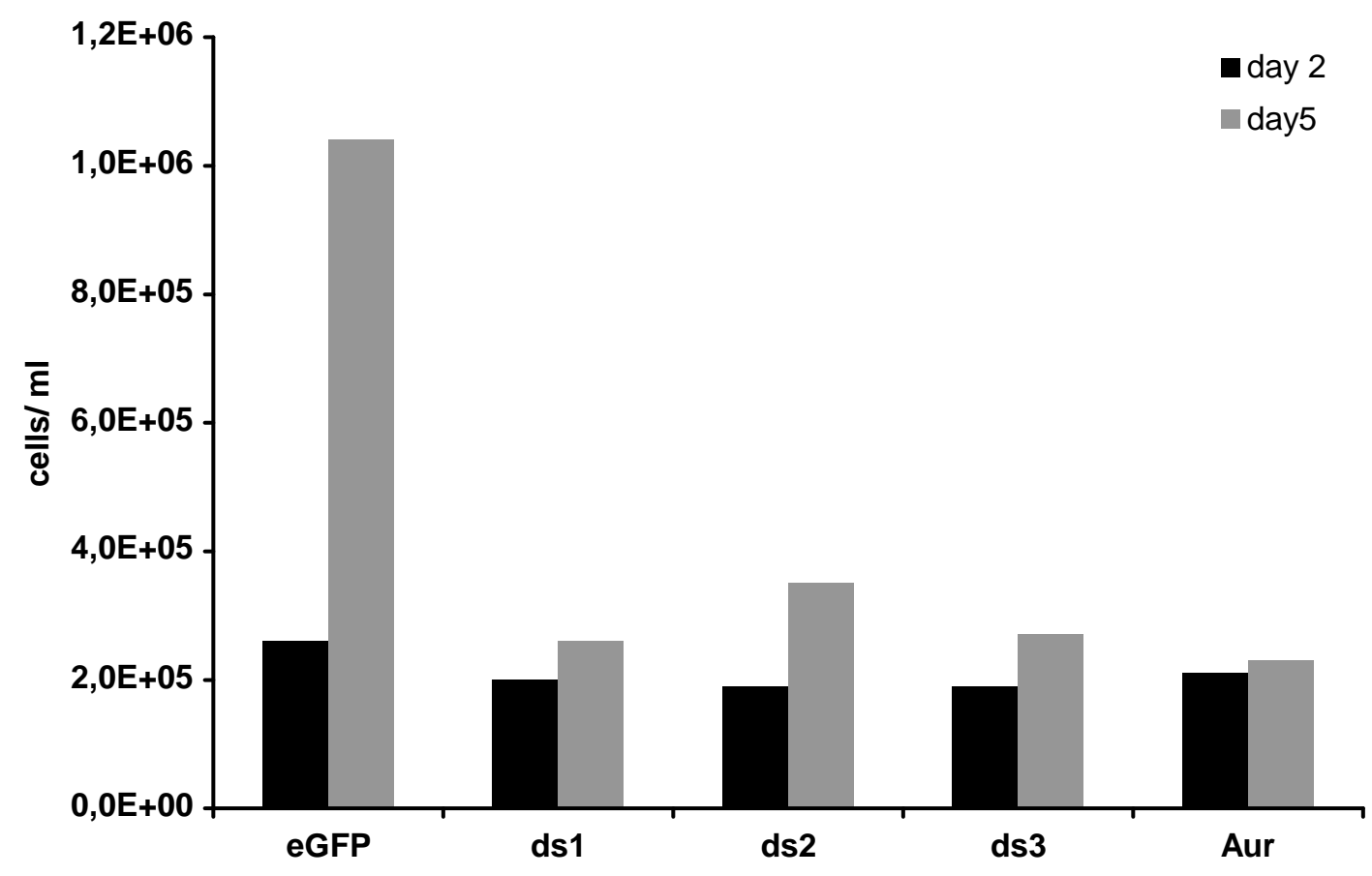

Figure 16: Proliferation of SL2 cells was reduced after 5 days of RNAi treatment with dsRNA against dTip60 (ds1-3) and Aur, but not with mock-treatment against eGFP. 
We concentrated on ds3 for further RNAi experiments and evaluated the intensity of dTip60-RNAi knock-down by ds3 with semiquantitative PCR and real-time PCR. ds3 effectively reduced dTip60 mRNA levels (Figure 17). Quantitative real-time PCR revealed that the level of dTip60 mRNA was knocked down to $8 \%$.

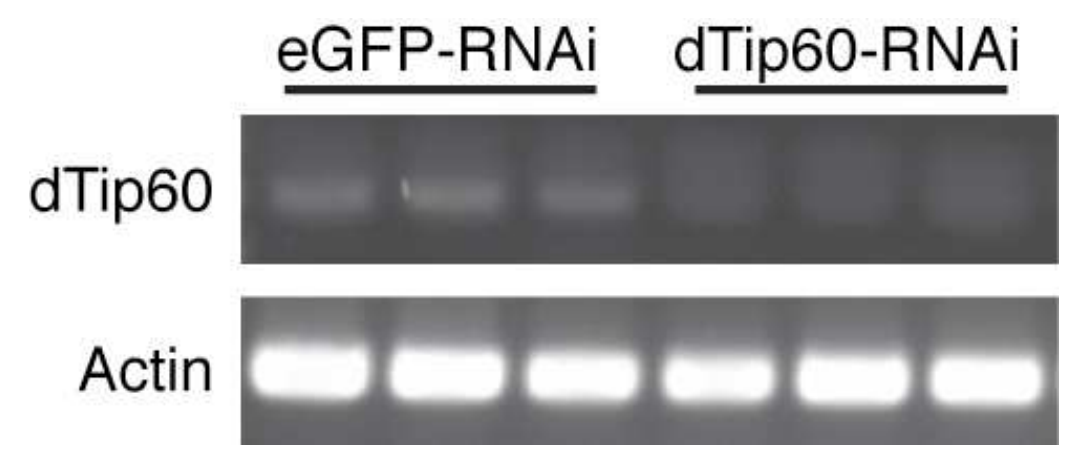

Figure 17: After five days of RNAi treatment with ds3, dTip60 mRNA was visibly reduced in comparison to Actin mRNA levels.

We next performed microarray-based expression analysis on three biological replica of a dTip60 knock-down and identified 1390 reliable measured targets that showed significant change of expression levels upon downregulation of dTip60 ( $<<0.05$, see Materials and Methods for details). Drosophila is currently predicted to have about 13800 genes. Therefore, our analysis indicated that roughly $10 \%$ of all genes in Drosophila SL2 cells were regulated by a dTip60-containing complex, which emphasized the central role of dTip60 in gene regulation. Of those 1390 genes, 785 were downregulated indicating that dTip60 was required for their activation. Surprisingly, 605 genes were upregulated in the absence of dTip60 (Figure 18). Change of gene expression could either be due to a direct function of dTip60 in activation or repression, or could be the consequence of indirect effects of dTip60, e. g. on the expression of transcription factors. 


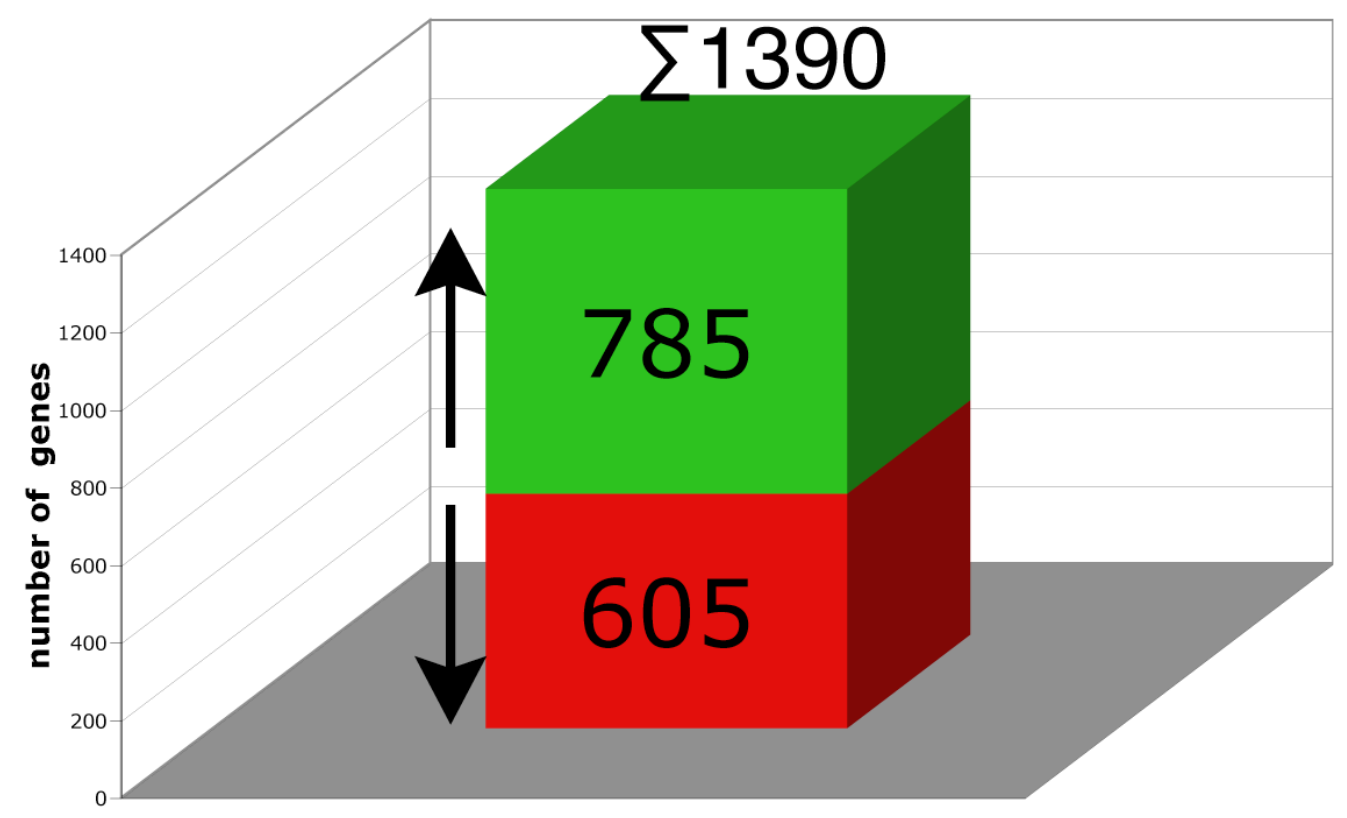

Figure 18: Genome-wide expression analysis revealed that 1390 genes were significantly regulated by dTip60, with 785 upregulating and 605 genes downregulating their expression upon loss of dTip60.

\section{6 dTip60-regulated genes were involved in chromatin-related processes}

We were interested in the biological function of the genes regulated by dTip60 and therefore performed an analysis of the Gene Ontology (GO) annotations of these potential target genes. The Gene Ontology assigns clearly defined terms describing biological functions to genes, which faciliates bioinformatical analysis of those terms.

Of the 1390 genes which significantly changed their expression upon dTip60 loss, 1123 were GO annotated. We asked whether there was an enrichment of a GO category among these genes and indeed found that there was a strong enrichment of genes with chromatin-related annotations (Table 2). This suggested that dTip60 plays an important role in the regulation of genes with chromatin-associated functions. 
Table 10: GO category association of genes regulated by dTip60 (with computational support by Dr. Ludger Klein-Hitpaß)

\begin{tabular}{|c|c|c|c|l|}
\hline GO category & $\begin{array}{c}\text { Total number } \\
\text { of genes in } \\
\text { category } \\
\text { dTip60-regulated } \\
\text { genes in category }\end{array}$ & $\begin{array}{c}\text { Number of } \\
\text { dT-value }\end{array}$ & Biological function \\
\hline $0006325 /$ & 302 & 140 & $<10^{-100}$ & $\begin{array}{l}\text { Establishment and/or } \\
\text { maintenance of chromatin } \\
\text { architecture/ DNA } \\
\text { packaging }\end{array}$ \\
\hline 0006333 & 240 & 130 & $<10^{-100}$ & $\begin{array}{l}\text { Chromatin assembly or } \\
\text { disassembly }\end{array}$ \\
\hline 0051276 & 391 & 167 & $<10^{-100}$ & $\begin{array}{l}\text { Chromosome organization } \\
\text { and biogenesis }\end{array}$ \\
\hline
\end{tabular}

\section{7 dTip60 acted as a repressor at distinct genes}

We further validated the effect of dTip60 on gene regulation by determining how the expression of the affected genes changed during the course of dTip60 depletion. We thus sought to gain insight into the dynamics of dTip60 mRNA loss and how immediate the response in expression levels of potential dTip60 target was. One might expect that direct targets of dTip60 respond shortly after the dTip60 mRNA decrease, whereas genes indirectly affected by dTip60 loss, for instance because of a loss in cell viability or changes in cell cycle progression, might show a delay in their downregulation.

To this end, we performed a time-course experiment of dTip60 knock-down. We measured cell proliferation and found that after three days of RNAi treatment, proliferation of treated cells stalled in comparison to mock-treated cells (Figure 19). 


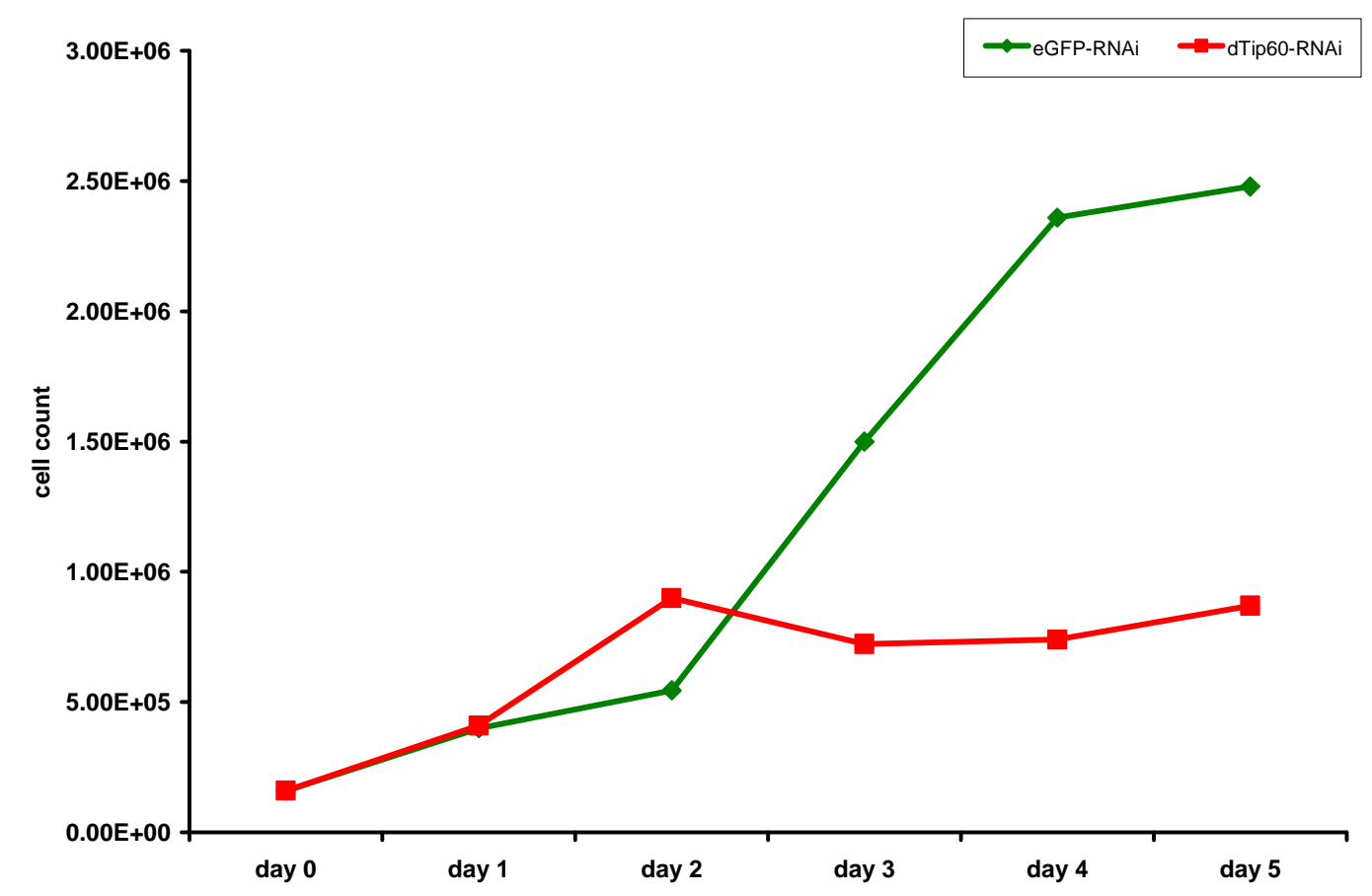

Figure 19: Proliferation of treated (dTip60-RNAi) and mock-treated (eGFP-RNAi) cells. Cell proliferation stalled after three days of dTip60 knock-down.

In order to assess changes of mRNA level during the time course of dTip60 depletion, RNAi-treated cells were harvested immediately and in intervals of 24 hours, and the expression of dTip60 and selected genes that served as representative examples from the microarray analysis were tested by reverse transcription and quantitative PCR.

We asked how dTip60 mRNA levels changed during dTip60 knock-down. Within 24 hours of RNAi depletion, the level of dTip60 mRNA dropped to approximately $14 \%$ (Figure 16). In the next two days, the dTip60 mRNA level was further reduced, and this level remained stable for the last two days of the time course experiment. The maximum level of dTip60 mRNA reduction occurred after 36 hours, with 7\% of dTip60 mRNA remaining, which was in good agreement with the depletion experiments used for the microarray analysis.

First, we evaluated the expression of genes that were identified as upregulated upon loss of dTip60 in the microarray analysis. A representative sample of seven genes was selected. In the microarray analysis, mthl 2 displayed a prominent upregulation with the $\log _{2}$ (dTip60 RNAi/ eGFP RNAi) above 4.85 in all three microarray experiments. CG10131 showed a moderate upregulation ( $\log _{2}$ (dTip60 RNAi/ eGFP RNAi) above 0.37). Fz2 was upregulated by a $\log _{2}$ (dTip60 RNAi/ eGFP RNAi) of more than 1.3. 
The other selected genes (CG14273, CG8942, CG5397, drip) displayed an intermediate upregulation, with their $\log _{2}$ (dTip60 RNAi/ eGFP RNAi) being above 2. All seven genes were present in both dTip60 knock-down and mock-treated cells and showed robust expression.

In the time course depleton of dTip60, six of these seven genes showed upregulation after two days of RNAi treatment (CG14273, CG8942, CG5397, drip, CG10131, mth12), and their expression further increased up to five days of treatment (Figure 20). Of these, mthl 2 showed the strongest upregulation, which is in accordance with the findings of the gene expression analysis. This suggested that these genes might be direct targets of dTip60. In contrast, the gene Fz2 showed a delayed upregulation after four days of RNAi treatment, suggesting that it might be indirectly affected by dTip60 loss.

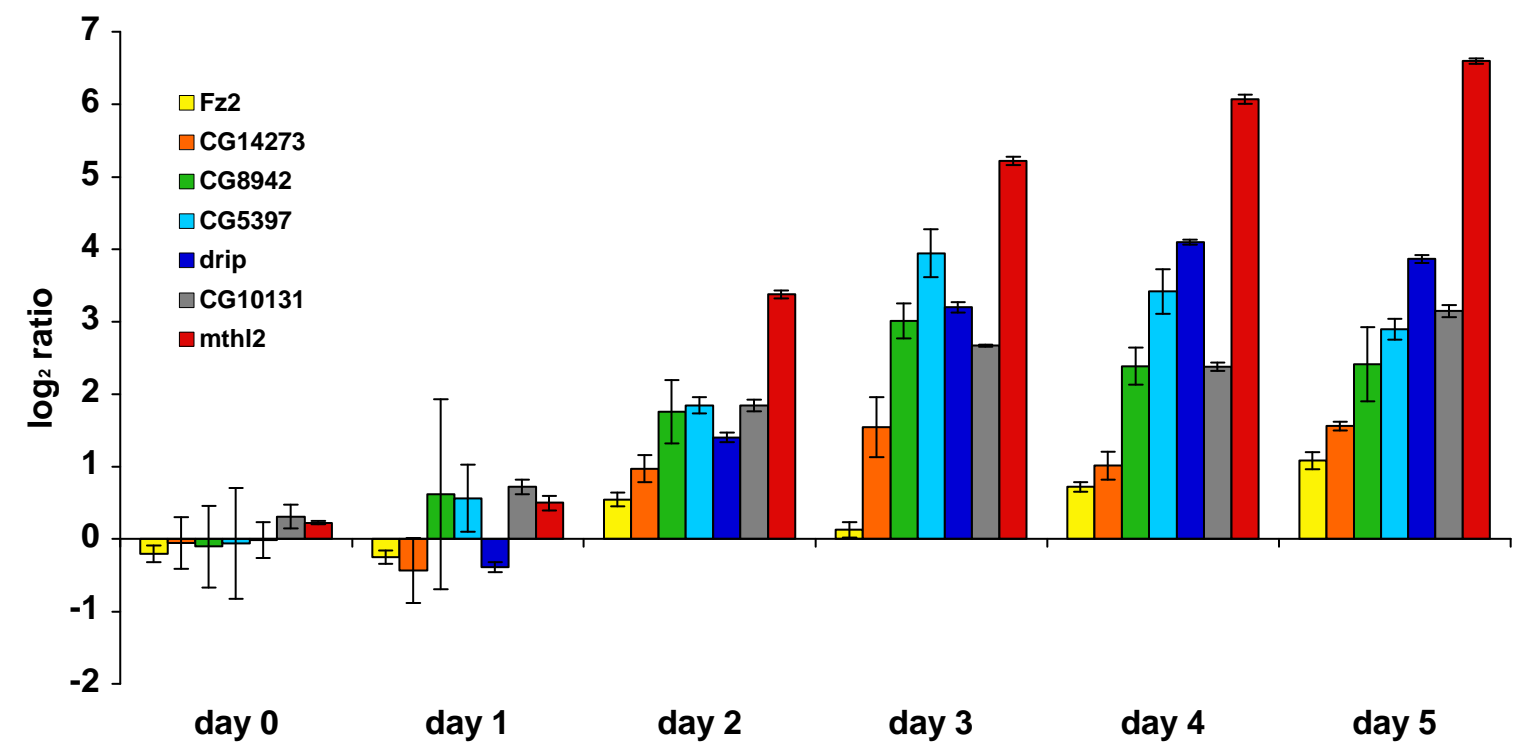

Figure 20: Upregulation of selected genes in SL2 cells after zero to five days of dTip60 RNAi treatment.

We next investigated the expression levels of genes that showed downregulation in the microarray analysis. Again, candidate genes were selected based on the magnitude of their change in gene expression and on whether they were present upon RNAi treatment against dTip60 or mock treatment. Ac3 displayed a moderate downregulation in all three microarray experiments, with its $\log _{2}$ (dTip60 RNAi/ eGFP RNAi) being below 0.5. All other selected genes (His4r, CG16888, nvy, CG11899) showed a more 
pronounced downregulation with a $\log _{2}$ (dTip60 RNAi/ eGFP RNAi) below -1.5 in all three microarray experiments.

The analysis of these candidates in the time course experiment gave a mixed picture. The gene most consistently downregulated was CG11899, though strong downregulation was only seen after prolonged RNAi treatment (Figure 16), implying that it might indirectly be affected by dTip60 reduction. Three other genes (Ac3, CG16888, nvy) showed a moderate level of downregulation that was apparent at later timepoints of RNAi treatment, again suggesting that they were not direct targets of dTip60. One gene, His4r, only showed downregulation after four days of RNAi treatment, but not at the other timepoints, which might explain why it appeared as regulated in the microarray analysis that was performed at this timepoint. Therefore, from this analysis it seemed unlikely that His4r was directly regulated by dTip60.

Taken together, this analysis suggested that the expression of six genes - CG8942, CG5397, CG10131, drip and mthl2 - increased as a result of dTip60 loss, suggesting a direct role for dTip60 in their repression. In contrast, the genes downregulated in the absence of dTip60 showed a moderate and delayed downregulation, implying that their changes may be due to indirect effects of dTip60, for instance on cell viability.

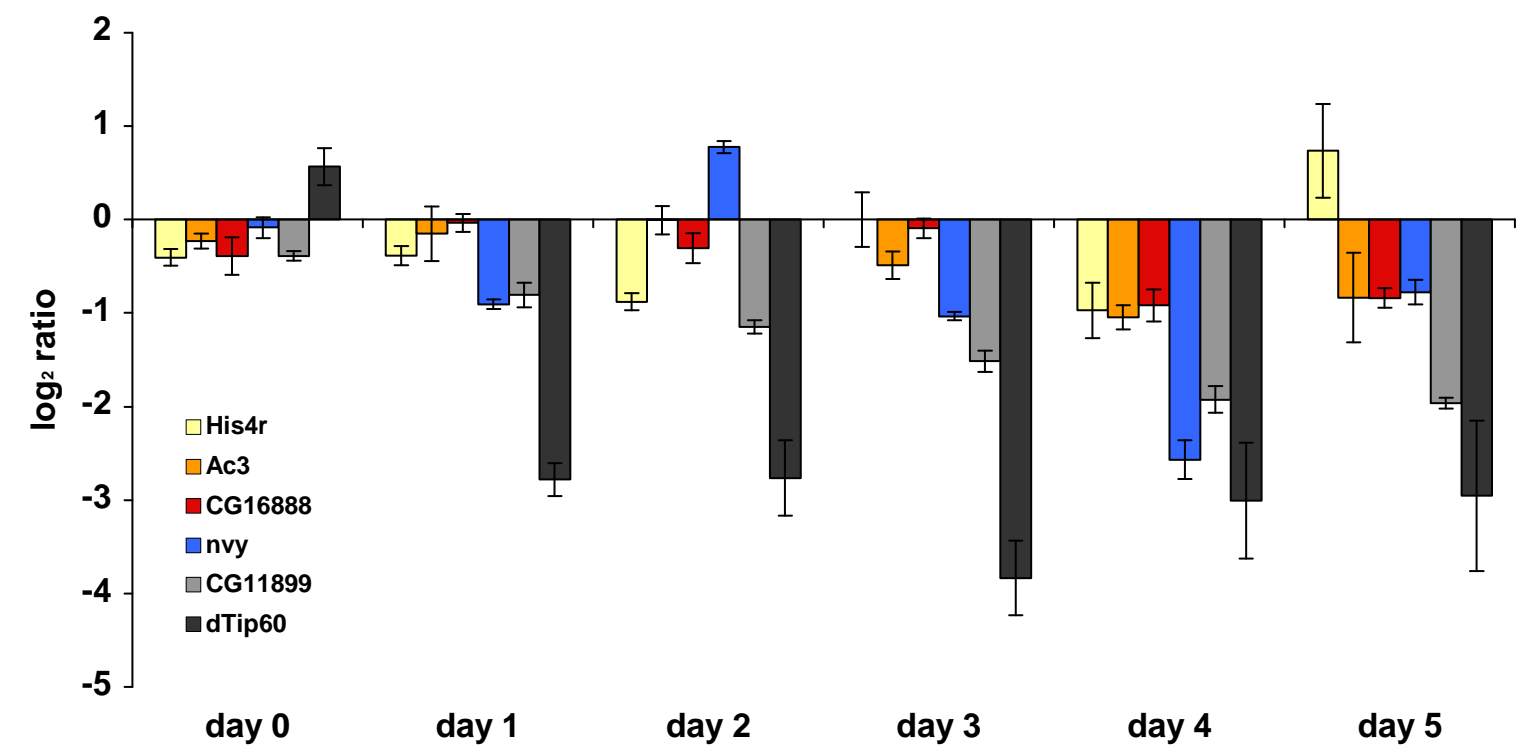

Figure 21: Downregulation of selected genes and dTip60 expression in SL2 cells after zero to five days of dTip60 RNAi treatment. 


\section{8 dTip60 was physically located at repressed genes}

The finding that dTip60 might have repressive function raises the question whether this effect is mediated directly or indirectly. Therefore, we asked if dTip60 was physically associated with the promotor region of these potential target genes. In order to test this, we measured the association of dTip60 with its target genes by chromatin immunoprecipitation analysis (ChIP). We performed ChIP with either dTip60 antiserum or with preimmunserum as a control. Promotor or ORFs of dTip60 target genes were tested, and an intergenic control region downstream of the 3' end of Hsp70 at the chromosomal location 87C served as a control. Significantly, dTip60 was enriched on the promotor sequences of the CG5397, drip, CG8942 and mthl2, whereas no enrichment was observed on an intergenic control region (Figure 22). Furthermore, dTip60 showed enrichment in the gene body of the CG5397 and drip genes. Taken together, this showed that dTip60 was directly located at the repressed genes, further indicating that its repressive effect was due to a direct function at these sequences.

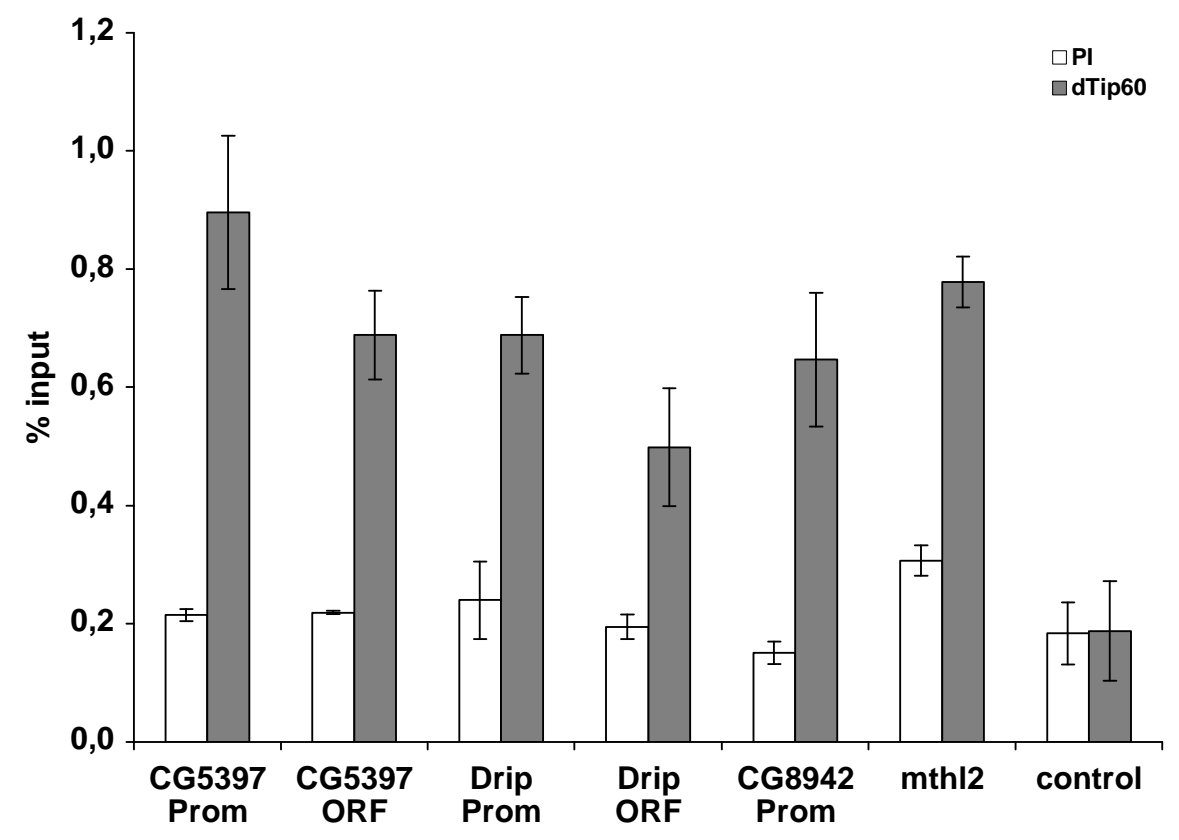

Figure 22: Localization of dTip60 protein at dTip60-regulated genes by ChIP analysis. dTip60 binding is shown as enrichment in ChIP experiments with the dTip60 antibody or preimmune serum (PI) relative to the input DNA. Error bars indicate standard deviations of three independent determinations. Prom, enrichment in promoter region; ORF, enrichment in the coding region of the respective gene. Control, enrichment on a fragment in the intergenic region downstream of the 3' end of Hsp70 at 87C (with technical help from Franziska Seifert) 


\subsection{Cooperation of dTip60 and HDAC1 in gene repression}

Histone acetyltransferases are generally thought to be involved in gene activation. However, our results suggested that dTip60 played a direct role in the repression of a number of genes in the embryonic SL2 cell line. In humans, there is evidence that hTip60 acts in a complex with a histone deacetylase, which mediates a repressive function (Ai et al., 2007; Xiao et al., 2003). Therefore, we hypothesized that in Drosophila, dTip60 might also work with an HDAC to repress distinct genes.

In order to test this hypothesis, we made use of the work of Foglietti et al, who performed genome-wide expression analysis of SL2 cells treated with Trichostatin A (TSA), which inhibits class I and II, but not class III HDACs, as well as individual RNAi knock-down of HDAC1-4 and HDAC X (Foglietti et al., 2006). First, we compared the genes upregulated or downregulated upon dTip60 loss to those upregulated or downregulated as a result of treatment with TSA. Importantly, there was a significant overlap between those group of genes $(\mathrm{p}<0.0002)$, and this overlap increased over the time of TSA treatment (Table 3). 118 genes that appeared upregulated after five days of TSA treatment were also identified as upregulated upon dTip60 loss. For downregulated genes, there was an overlap of 109 genes (Table 4).

The respective $\mathrm{p}$ values $\left(5.15 \times 10^{-42}\right.$ for upregulated genes, and $6.08 \times 10^{-48}$ for downregulated genes) indicate that these findings are statistically highly significant. These results strongly supported the notion that dTip60 cooperates with a TSA-sensitive HDAC to regulate gene expression.

We next sought to determine which HDAC might cooperate with dTip60 in gene repression. Foglietti et al. performed genome-wide expression analysis upon HDAC1, 2, 3, 4 and $\mathrm{X}$ depletion, but surprisingly only found significant changes in gene expression for HDAC1 and HDAC3 knock-down (Foglietti et al., 2006). We therefore compared our expression data to the effect of depleting those two HDACs. Interestingly, there was a significant overlap between genes upregulated by dTip60 and HDAC1, but not HDAC3 (Table 3). This suggested that dTip60 and HDAC1 cooperated in gene repression, perhaps by histone deacetylation in the promoter region of genes. In addition, the overlap in gene expression signatures between dTip60 and HDAC1 extended to genes downregulated by the two factors (Table 4), implying that they not only cooperated in gene repression, but also in gene activation. 
Table 11: Comparison of genes upregulated upon dTip60 depletion with TSA treatment and loss of HDAC1 or HDAC3 as determined by Foglietti et al. Analysis was performed with scientific support from Prof. Daniel Hoffmann.

\begin{tabular}{|c|c|c|c|}
\hline \multicolumn{4}{|c|}{ Genes upregulated upon dTip60 loss $(\mathrm{n}=605)$} \\
\hline & $\begin{array}{c}\text { Total genes } \\
\text { upregulated by } \\
\text { treatment }\end{array}$ & $\begin{array}{l}\text { Overlap with } \\
\text { dTip60 }\end{array}$ & $\mathrm{P}$-value \\
\hline $\begin{array}{l}\text { TSA } \\
12 \mathrm{~h}\end{array}$ & 352 & 31 & 0.0002 \\
\hline $\begin{array}{l}\text { TSA } \\
24 \mathrm{~h}\end{array}$ & 310 & 40 & $5.72 \times 10^{-10}$ \\
\hline $\begin{array}{l}\text { TSA } \\
3 \text { days }\end{array}$ & 407 & 69 & $4.72 \times 10^{-23}$ \\
\hline $\begin{array}{l}\text { TSA } \\
5 \text { days }\end{array}$ & 660 & 118 & $5.15 \times 10^{-42}$ \\
\hline $\begin{array}{l}\text { HDAC1 } \\
3 \text { days }\end{array}$ & 138 & 21 & $4.88 \times 10^{-07}$ \\
\hline $\begin{array}{l}\text { HDAC1 } \\
5 \text { days }\end{array}$ & 494 & 55 & $5.54 \times 10^{-31}$ \\
\hline $\begin{array}{l}\text { HDAC3 } \\
3 \text { days }\end{array}$ & 6 & 0 & 1 \\
\hline $\begin{array}{l}\text { HDAC3 } \\
5 \text { days }\end{array}$ & 29 & 4 & 0.0351 \\
\hline
\end{tabular}


Table 12: Comparison of genes downregulated upon dTip60 depletion with TSA treatment and loss of HDAC1 or HDAC3 as determined by Foglietti et al. Analysis was performed with scientific support from Prof. Daniel Hoffmann.

\begin{tabular}{|c|c|c|c|}
\hline \multicolumn{4}{|c|}{ Genes downregulated upon dTip60 loss $(n=785)$} \\
\hline & $\begin{array}{c}\text { Total genes } \\
\text { downregulated by } \\
\text { treatment }\end{array}$ & $\begin{array}{c}\text { Overlap with } \\
\text { dTip60 }\end{array}$ & P-value \\
\hline $\begin{array}{l}\text { TSA } \\
12 \mathrm{~h}\end{array}$ & 237 & 43 & $6.42 \times 10^{-12}$ \\
\hline $\begin{array}{l}\text { TSA } \\
24 \mathrm{~h}\end{array}$ & 255 & 68 & $2.97 \times 10^{-28}$ \\
\hline $\begin{array}{l}\text { TSA } \\
3 \text { days }\end{array}$ & 218 & 75 & $1.59 \times 10^{-39}$ \\
\hline $\begin{array}{l}\text { TSA } \\
5 \text { days }\end{array}$ & 385 & 109 & $6.08 \times 10^{-48}$ \\
\hline $\begin{array}{l}\text { HDAC1 } \\
3 \text { days }\end{array}$ & 39 & 6 & 0.0206 \\
\hline $\begin{array}{l}\text { HDAC1 } \\
5 \text { days }\end{array}$ & 338 & 86 & $6.50 \times 10^{-34}$ \\
\hline $\begin{array}{l}\text { HDAC3 } \\
3 \text { days }\end{array}$ & 4 & 0 & 1 \\
\hline $\begin{array}{l}\text { HDAC3 } \\
5 \text { days }\end{array}$ & 35 & 5 & 0.0444 \\
\hline
\end{tabular}




\section{Discussion}

The MYST histone acetyltransferase dTip60 is part of a multimeric protein complex that unites both HAT and chromatin remodeling activites. In this study, we characterized the properties of dTip60 and evaluated the function of dTip60 in gene regulation in the model organism Drosophila melanogaster.

We found that strong reduction of dTip60 by RNAi was lethal, whereas a moderate knock-down led to defects in wing development. Reduction of dTip60 in the wing imaginal disc globally increased methylation of H3 K27 and caused increased apoptosis. Furthermore, dTip60 was present in the nucleus of Drosophila embryos and was localized to about $50 \%$ of interbands on polytene chromosomes. Genome-wide expression analysis showed that dTip60 regulated roughly $10 \%$ of all genes in Drosophila SL2 cells, and that many of these genes had chromatin-related functions. Interestingly, reduction of dTip60 levels caused repression at some genes, but activation at others, implying a role for dTip60 both in gene activation and repression. Furthermore, dTip60 was physically present at repressed genes, suggesting a direct role in their repression. Statistical analysis of expression signatures indicated that dTip60 worked in cooperation with HDAC1 in gene repression.

\section{1 dTip60 was essential for viability in flies}

We demonstrated that dTip60 is essential in flies with the help of a dTip60-RNAi fly line using the UAS/Gal4 system. This was in good agreement with previous work (Zhu et al., 2007), where a different UAS/Gal4 dTip60-RNAi line displayed early pupal lethality under the control of the strong and ubiquitous Gal4-driver Act5c. We tested different Gal4 driver lines at different temperatures in order to modulate dTip60 RNAi knock-down efficiency. Strong knock-down was lethal, whereas moderate knock-down lead to the formation of ectopic wing veins, which can be considered a relatively mild phenotype. Therefore, there seems to be a relatively narrow window for the reduction of dTip60 protein: If the amount of dTip60 protein that causes a mild phenotype is only slightly reduced, this results in lethality.

Why does lethality occur in the late pupae? A combination of three effects might allow flies to survive up until this relatively late stage: first, due to the maternal effect, some 
dTip60 protein might be passed on from the mother to the progeny; second, knockdown efficiency might leave dTip60 protein levels just high enough for flies to live until pupae; and third, lack of dTip60 protein might have the highest impact on processes needed no earlier that the late pupae. Our current state of knowledge does not allow a distinction between these possibilities.

In the embryonic Drosophila cell line SL2, knock-down of dTip60 led to reduced proliferation. This suggests that dTip60 was essential on a cellular level and is in agreement with previous findings (Zhu et al., 2007), where dTip60-RNAi caused cellular defects and lethality in SL2 cells.

Not all MYST HATs are essential: Sas2 in S. cerevisiae is not essential (EhrenhoferMurray et al., 1997), and lack of MOF in D. melanogaster is lethal in males, but not females due to its role in dosage compensation (Akhtar and Becker, 2000). Genomewide analysis revealed that in SL2 cells, dTip60 regulated directly and indirectly about $10 \%$ of all genes in Drosophila, indicating that dTip60 is involved in a wide range of biological functions. Significant disturbance in gene expression of a high percentage of genes might lead to biological effects that negatively affect viability.

Among this wide range of biological functions influenced by dTip60, some could be identified in this work. For example, dTip60 showed an effect on chromatin related functions, such as chromatin assembly and disassembly and DNA packaging, by regulating about half of all genes with this GO annotation. Chromatin structure is highly important for gene expression, replication, recombination and repair. If loss of dTip60 negatively affects the integrity of chromatin structure, this might lead to cell death.

\subsection{Effects of dTip60 on wing development}

In our assay, dTip60 had an effect on wing vein development in that reduction of dTip60 protein led to the formation of ectopic wing veins parallel to L5 or, with reduced frequency, at other positions of the wing.

There are two possible models for how dTip60 might influence wing development. During development, at least five different signaling pathways contribute to the establishment of vein and intervein territories: Hedgehog (Hh) signaling, bone morphogenetic protein (BMP) signaling, epidermal growth factor receptor (Egfr) signaling, Wingless (Wg) and Notch $(\mathrm{N})$ signaling (Blair, 2007). dTip60 might be affecting any of these pathways, which would result in disturbed vein formation in the 
absence of dTip60. The genetic complexity of wing development makes it difficult to identify the possible pathway affected by dTip60 loss.

It is also possible that the formation of ectopic wing veins in dTip60-RNAi flies is due to a silencing defect. During evolution, a variety of wing patterns evolved in insects, and most insects display about twice the number of veins as Drosophila. This fact has led to the hypothesis that in Drosophila, a subset of veins are silenced. This theory is supported by the fact that with high frequency, ectopic veins run longitudinally between normal veins (Bier, 2000). Therefore, the short ectopic vein running parallel to L5 might be a silencing defect as a consequence of derepression by loss of dTip60.

Other evidence supports the notion that, in other contexts, dTip60 plays a role in silencing. Ai and colleagues propose that the dTip60 complex promotes the generation of a repressive chromatin state via its subunit Reptin (Qi et al., 2006). A recent study demonstrated a cooperation of the dTip60 complex with RSF and suggested that those two work together at silent chromatin formation (Hanai et al., 2008).

The wing phenotype caused by dTip60 reduction prompted us to investigate the consequences of dTip60 protein reduction in the imaginal wing disc. We found that reduction of dTip60 caused increased H3K27 trimethylation, which has been correlated with transcriptional silencing. In Drosophila, trimethylation of H2K27 is introduced by the PRC2 complex, which is a Polycomb Group (PcG) protein complex. (Czermin et al., 2002; Muller et al., 2002). PcG proteins are evolutionary conserved proteins required for maintaining long-term repression of genes. The finding that trimethylation of H3K27, a repressive mark, is enriched if dTip60 protein levels are reduced suggests dTip60 might act as a counterplayer of the PRC2 complex in maintaining gene expression of a subset of genes. Since our analysis showed that dTip60 is required for the activation as well as the repression of a large number of genes in the Drosophila genome, this suggests that dTip60 is not restricted to just one role in either activation or repression.

Further analysis of imaginal wing disc revealed that reduction of dTip60 protein levels caused increased apoptosis. This indicated that dTip60 was needed in this tissue at this point of time in development. The finding that dTip60 is essential for cells of imaginal wing discs is in line with the requirement of dTip60 for viability in flies and SL2 cells. 


\section{3 dTip60 in transcriptional activation}

dTip60 is part of a dual-activity HAT/ chromatin remodeler complex (Squatrito et al., 2006). Generally, one might posit a role for such a complex in gene activation. Chromatin is repressive, and this repression needs to be overcome by histone acetylation and chromatin remodeling at the gene promoters, which thus help in the recruitment of the transcriptional machinery. In the context of transcriptional activation, histone acetylation in the proximity of promotor regions serves as a signal for the recruitment of chromatin remodeling factors, which then mobilize nucleosomes in the proximity of the promotor.

This prominent role for HAT and chromatin remodeler complexes in gene activation prompted us to ask whether the dTip60 complex plays a role in transcriptional activation.

Genome-wide transcription analysis showed that upon loss of dTip60, many genes downregulated their expression, suggesting that indeed, dTip60 is required for the activation of a large number of genes in the Drosophila genome. This activation may be due to the direct effect of the dTip60 complex performing histone acetylation and chromatin remodeling. Alternatively, activation of transcriptional gene activity might also be independent of HAT activity (Gavaravarapu and Kamine, 2000), for example by acetylation of non-histone proteins such as transcription factors.

In addition to an activating function of dTip60 as evaluated by genome-wide transcriptional analysis, two other findings also support the notion that dTip60 has an activation function. As discussed preiviously, increased H3K27 trimethylation suggested a role for dTip60 in gene activation as a counterplayer of PRC2. Also, dTip60 was localization to interbands on polytene chromosomes, a finding that is consistent with a role in gene activation and will be discussed in the following paragraph. 


\subsection{Function and localization of dTip60 on polytene chromosomes}

In Drosophila, polytene chromosomes are present in the salivary glands of third instar larve and provide an useful model for studies of chromosomes and chromatin domains. As a result of differential chromatin compaction, polytene chromosomes are organized in bands and interbands. Heterochromatic bands are thought to be associate with inactive genes, whereas transcription mostly occurs in the relatively decondensed interbands.

We demonstrated that on polytene chromosomes, dTip60 localized to a subset of interbands, suggesting an euchromatic association of dTip60 and role for dTip60 in gene activation. This is consistent with our finding in SL2 cells, where dTip60 is involved in the activation of a large number of genes.

In order to establish and maintain band and interband structure, they are separated by boundaries. It has been demonstrated that insulator proteins are localized to these boundaries, where they are thought to prevent spreading of heterochromatin in euchromatic regions (Dorman corces). We found that dTip60 also localized to the border of interbands on polytene chromosomes, which is compatible with a barrier function for dTip60. However, the pattern of dTip60 in these regions does not overlap with the pattern of known insulator proteins such as BEAF-32 and $\operatorname{Mod}(\operatorname{mdg} 4)$.

\section{5 dTip60 in transcriptional repression}

In addition to a role for dTip60 in gene activation, our data also reveal a substantial contribution of dTip60 to gene repression. Genome-wide trancriptional analysis revealed that dTip60 has a repressive effect on a large amount of genes. We asked whether this repression was mediated directly by dTip60 or was an indirect effect as a consequence of dTip60-regulated proteins with a regulatory function in transcription. dTip60 was physically located at the promotor regions of repressed genes, suggesting that dTip60 has a direct role in gene repression.

It has been shown previously in several studies in humans and mice that despite the prominent role of histone acetyltransferases in gene activation, Tip60 can have repressive function. For example, hTip60 was found to act as a corepressor by 
recruitment of HDAC7 (Xiao et al., 2003) and represses HDC promotor activity together with KLF4 and HDAC7 (Ai et al., 2007).

In Drosophila, how does the HAT dTip60 mediate its repressive function?

\subsection{1 dTip60 might mediate its repressive function by deposition of $\mathrm{H} 2 \mathrm{Av}$}

The dTip60 complex acetylates phosphorylated H2A.v and replaces it with the unmodified form (Kusch et al., 2004). H2Av in Drosophila is present at heterochromatin and is involved in the formation of repressed chromatin. Since phospho-H2Av is not only associated with DNA damage repair, but also with general transcriptional activation in flies (Swaminathan et al., 2005), one might then speculate that the dTip60 complex is globally required to deposit $\mathrm{H} 2 \mathrm{Av}$ on repressed genes. Loss of dTip60 would therefore result in a loss of H2Av and hence a loss of gene repression. Notably, one homolog of dTip60, Sas2 in S. cerevisiae, has also been implicated in gene silencing at yeast telomeres and the silent mating-type loci (Ehrenhofer-Murray et al., 1997; Meijsing and Ehrenhofer-Murray, 2001; Reifsnyder et al., 1996). However, Sas2's telomeric silencing function stems from its role as a boundary factor in preventing the spreading of heterochromatin into euchromatic regions (Kimura et al., 2002; Suka et al., 2002), rather than from direct promoter acetylation. In addition, the boundary function of Sas 2 may entail the deposition of H2A.Z in subtelomeric regions (Shia et al., 2006). Therefore, by analogy, dTip60 may also act in gene repression in flies as a boundary factor by restricting heterochromatin to its designated genomic region. In line with this, dTip60 localized to the border of interbands on polytene chromosomes, which is compatible with a barrier function for dTip60. However, our ChIP analysis showed localization of dTip60 at repressed genes, arguing for a more direct role of dTip60 in gene repression. As discussed before, the interband localization of dTip60 is also consistent with its role in gene activation. 


\subsubsection{The repressive function of dTip60 might be independent of its HAT function}

Another possibility for dTip60's role in gene repression is that it is not the HAT function of dTip60 that is relevant for repression. Notably, Tip60 can acetylate histones in the nucleosomal context only when it is within a protein complex, but not as a recombinant protein (Ikura et al., 2000), suggesting that other accessory proteins (and perhaps chromatin remodelling) are required for its activity. In consequence, the activity of these factors may be more important for the repressive activity of the dTip60 complex than the HAT activity alone, for instance by moving nucleosomes over the promoter of genes to a position repressive to transcription.

The study by $\mathrm{Ai}$ and Wang adds a mechanistic view to a function of dTip60 independent of its HAT activity. The authors demonstrated that Tip60 physically interacts with HDAC7, and that the HDAC7-interacting domain of Tip60 displayed great overlap with the region required for HAT activity (Ai et al., 2007). This finding led to the hypothesis that the Tip60 complex can change its properties from a trancriptional activator to a transcriptional repressor, with the repressive function mediated by HDAC activity and HAT activity lost upon binding of HDAC7.

dTip60 contains a chromodomain, a zinc finger and a HAT domain. The function of dTip60 therefore may not only lie in its ability to acetylate histones, but also in the recruitment of other catalytically active complex components via its other protein domains. Also, acetylation of non-histone proteins, especially transcription factors, might be a strategy for Tip60-mediated expression.

\subsection{3 dTip60 might cooperate with HDAC1 in gene regulation}

Our data indicated that dTip60 cooperates with HDAC1 in gene regulation at a subset of genes, although we see this correlation for genes both up- and down-regulated by dTip60 and HDAC1, implying that they cooperate in gene activation as well as in repression. Therefore, the dTip60 complex may recruit HDAC1 to the promoters to perform histone deacetylation, which then causes gene regulation. The final transcriptional output of a gene may then result from the sum of (histone) acetylation by dTip60 and deacetylation by HDAC1. In support of this cooperation of dTip60 and HDAC1, mutations in HDAC1, like mutations in dTip60 components, cause defects in 
position effect variegation (Mottus et al., 2000). Furthermore, genes that are weakly activated by dTip60/ HDAC1 may be in a "poised" state of gene expression and carry both transcription-activating and -repressing chromatin marks. Such genes could then be rapidly and strongly activated under inducing conditions.

For the regulation of genes by dTip60 that are independent of HDAC1, dTip60 most likely cooperates with other co-repressors. For some such genes, dTip60 may work with another TSA-sensitive HDAC, because the number of TSA-regulated genes was larger that the number of HDAC1-regulated genes. For other groups of genes, dTip60 probably collaborates with other, TSA-resistant HDACs, as well as with other, as yet unidentified co-repressors to control gene expression.

An alternative explanation for dTip60's role in gene repression is that dTip60 acetylates targets other than histones, and that these are relevant for dTip60's repressive function, whereas histone acetylation might be important for gene activation. Tip60 in known to acetylate other factors, for instance p53, which modulates its transcriptional output (Sykes et al., 2006; Tang et al., 2006).

\subsection{4 the function of dTip60 might be context-dependent}

dTip60 joins a growing number of chromatin regulators that have a context-dependent influence on gene expression in that they contribute both to gene activation and repression, although it remains to be determined how many of the dTip60 targets identified here are directly regulated by dTip60. Notably, the Drosophila MYST HAT Chameau stimulates transcription that depends on the transcription factor AP-1, and this coactivation is accompanied by increased histone acetylation at the gene promoter. (Miotto et al., 2006). Conversely, mutations in Chameau cause defects in position effect variegation and in the maintenance of Polycomb-dependent gene silencing, suggesting a parallel role for Chameau in gene repression (Grienenberger et al., 2002). Similarly, the dTip60 homolog MOF is best known for upregulating expression of X-linked genes in male flies through acetylation of H4 K16 (Mendjan and Akhtar, 2007). However, MOF is also required for the downregulation of a substantial number of genes on the autosomes of male and female flies (Kind et al., 2008). Furthermore, pericentric heterochromatin in Drosophila contains H4 K12 acetylation that depends on the presence of H2A.v, suggesting a role for this modification in establishing pericentric gene silencing (Swaminathan et al., 2005). Thus, there seems to be no simple 
correlation between the enzymatic activity of a regulator and its effect on gene expression at an individual genomic location. The overall transcriptional outcome likely depends on which cofactors interact with dTip60 at a given promoter, and what the sum of epigenetic modifications is. HDAC7, for example, displays a tissue-specific expression pattern (Kao et al., 2000) and its localization is signal-regulated (Fischle et al., 2001), which supports the idea of different dTip60-interacting partners in different tissues. 


\subsection{Summary and outlook}

In this study, we sought to gain insight into the biological functions of the MYST histone acetyltransferase dTip60, which is part of a multimeric protein complex. First, we evaluated the effects of dTip60 reduction by RNAi in flies. We found that on an organismal level, strong reduction of dTip60 caused lethality, whereas moderate reduction of dTip60 led to the formation of ectopic wing veins as well as increased apoptosis and trimethylation of H3K27 in the imaginal wing disc. Characterization of dTip60 localization revealed dTip60 located to the nucleus in early embryos and on interbands of polytene chromosomes

We wanted to further investigate the role of dTip60 in gene regulation. Genome-wide expression analysis demonstrated that dTip60 significantly regulated about $10 \%$ of all genes in Drosophila SL2 cells. Genes with chomatin-related functions were highly enriched among those genes regulated by dTip60. Surprisingly, our results suggested a role for dTip60 in both gene activation and repression. We demonstrated that dTip60 was physically present at promotor regions of repressed genes, suggesting a direct role for dTip60 in gene repression. We asked how this repressive function is mediated, and found a highly statistically significant overlap of gene expression profiles between dTip60 and HDAC1, suggesting that they cooperate in gene regulation.

With this work, we have shown a central role for dTip60 in both gene activation and repression in flies. Further characterization of this function of dTip60 is of high interest and will provide important insights into the genome-wide regulation of gene expression. Our results suggest an interaction of HDAC1 and dTip60 in gene regulation based on a comparison of gene expression signatures. In the future, it will be of interest to gain further insight into this proposed interaction. Do HDAC1 and dTip60 interact biochemically? Coimmunoprecipitation of dTip60 and HDAC1 would be the experiment of choice to test for an interaction. Furthermore, one might expect partial colocalization of dTip60 and HDAC1 on polytene chromosomes.

We showed that dTip60 is involved in the regulation of about $10 \%$ of all genes in Drosophila. For a small sample of repressed genes, we demonstrated that this effect was mediated directly by dTip60. On a global scale, it would be highly interesting to provide

a genome-wide map of dTip60 occupancy. Chromatin immunoprecifitation of dTip60 protein followed by microarray analysis could give insight into promotor occupancy by dTip60, therefore indicating which genes are directly regulated by dTip60. A similar 
experiment could be performed with HDAC1 and would provide information about which genes are jointly regulated by dTip60 and HDAC1. Our data suggest the possiblity that dTip60 and HDAC1 might also have functions in gene regulation independently of each other. Knowledge of genome-wide promotor occupancy by dTip60 and HDAC1 could answer the question which genes are regulated by either protein independently of the other. Does dTip60 regulate these genes in cooperation with other protein complexes? Is there a preference for an interaction of dTip60 with a certain protein complex in gene activation versus gene repression? A genome-wide map of dTip60 occupancy could also give more insight into the biological functions of dTip60, for instance whether dTip60 regulates pathway components or key proteins of developmental processes.

On a biochemical level, it would be of high interest if and what kind of biochemical modifications dTip60 might perform at promoter regions. One can assume that dTip60 acetylates histones in order to regulate gene expression. Which kind of histone modifications are introduced by dTip60? Is there a difference in the pattern of histone modification between genes activated and repressed by dTip60? However, the interpretation of such modification patterns may be complex due to the potential interaction of dTip60 with HDAC1, which may itself alter modification patterns.

This study highlighted the prominent role of dTip60 in epigenetic gene regulation. Further work is necessary to characterize the functions of dTip60 in detail. This is highly desireable, as it adds further knowledge to epigenetic processes, which play a central role in development, differentiation and disease. For example, the human homologue of dTip60, hTip60, is involved in acute myeloid leukemia. As the relevance of HDAC inhibitors as potent drugs in the treatment of cancer is emerging, characterization of interactions between Tip60 and HDACs is becoming highly medically relevant. Therefore, gaining knowledge about the function of dTip60 is not only a valuable contribution to science in general, but might also find medical application. 


\section{Abstract}

Epigenetic changes are crucial for the development and differentiation of cells and organisms. MYST histone acetyltransferases play an important role in the introduction of epigenetic modifications.

Here, we sought to gain insight into the biological functions of dTip60. We evaluated the consequences of dTip60 reduction and found that strong ubiquitous dTip60 knockdown in flies was lethal. Moderate knock-down of dTip60 in the wing led to developmental defects. In the imaginal wing disc, reduction of dTip60 caused increased tri-methylation of H3K27 and increased apoptosis. We characterized the localization of dTip60 in early embryos and found that it localized to the nucleus. On polytene chromosomes, dTip60 localized to the interbands, which suggests a role for dTip60 in gene activation.

We further investigated the role for dTip60 in gene regulation and performed genomewide expression analysis upon dTip60 depletion in cell culture. dTip60 regulated approximately $10 \%$ of all genes in Drosophila, among which genes with chromatinrelated functions were highly enriched. Surprisingly, about half of these genes were upregulated upon dTip60 loss, indicating that dTip60 has repressive as well as activating functions. We asked whether this repressive function of dTip60 was a consequence of a direct or indirect effect. dTip60 protein was directly located at promotor regions of a subset of repressed genes, suggesting a direct role in gene repression. How does the histone acetyltransferase dTip60 mediate this repressive function? We compared the gene expression signature of dTip60 with that of HDACs and found a significant correlation with the gene expression signature of HDAC1. This suggested that the dTip60 complex recruits an HDAC1-containing complex to regulate gene expression in the Drosophila genome. 


\section{Zusammenfassung}

Epigenetische Veränderungen sind von großer Wichtigkeit für die Entwicklung und Differenzierung von Zellen und Organismen. MYST Histonacetyltransferasen spielen eine zentrale Rolle bei der Einführung epigenetischer Modifikation.

Ziel dieser Arbeit war es, Einblicke in die biologischen Funktionen von dTip60 zu gewinnen. Es wurde mittels RNAi untersucht, zu welchen biologischen Konsequenzen eine Reduktion von dTip60 in Drosophila melanogaster führt. Starker knock-down von dTip60 war lethal, während ein moderater knock-down von dTip60 einen Flügelphänotyp verursachte. In Flügelimaginalscheiben führte eine Reduktion von dTip60 zu einer Zunahme von Trimethylierung an H3K27 und zu erhöhter Apoptose. Die Lokalisation von dTip60 im frühen Embryo wurde bestimmt. dTip60 ist am Zellkern lokalisiert. In polytänen Chromosomen ist dTip60 an den Interbanden lokalisiert, was auf eine Rolle von dTip60 in der Genaktivierung hindeutet.

Es wurde weiterhin die Rolle von dTip60 in der Genregulation untersucht, indem eine genomweite Expressionsanalyse von dTip60-RNAi behandelten SL2 Zellen durchgeführt wurde. Dabei hat sich gezeigt, dass dTip60 ungefähr $10 \%$ aller Drosophilagene reguliert. Unter den von dTip60 regulierten Genen waren solche mit einer Funktion in der Regulierung der Chromatinstruktur signifikant angereichert. Die genomweite Expressionsanalyse hat weiterhin ergeben, dass dTip60 nicht nur an der Genaktivierung, sondern auch an der Genrepression beteiligt ist. Um zu ermitteln, ob es sich dabei um einen direkten oder indirekten Effekt handelt, wurde überprüft, ob dTip60 in der Promoterregion reprimierter Gene vorhanden ist. Dies konnte für mehrere Gene gezeigt werden, was darauf hindeutet, dass dTip60 direkt an der Genrepression beteiligt ist. Wie aber kann eine Histonacetyltransferase eine reprimierende Funktion vermitteln? Es wurde untersucht, ob es eine Überlappung von dTip60 regulierten Genen mit solchen gibt, die von HDACs reguliert werden. Es hat sich gezeigt, dass es eine signifikante Korrelation von dTip60- und HDAC1-regulierten Genen gibt, was darauf hindeutet, dass ein HDAC1-Komplex mit dem dTip60-Komplex bei der Genregulation zusammenarbeiten könnte. 


\section{References}

Adelman, K., Wei, W., Ardehali, M. B., Werner, J., Zhu, B., Reinberg, D. and Lis, J. T. (2006). Drosophila Paf1 modulates chromatin structure at actively transcribed genes. Mol Cell Biol 26, 250-60.

Ai, W., Zheng, H., Yang, X., Liu, Y. and Wang, T. C. (2007). Tip60 functions as a potential corepressor of KLF4 in regulation of HDC promoter activity. Nucleic Acids Res 35, 6137-49.

Akhtar, A. and Becker, P. B. (2000). Activation of transcription through histone $\mathrm{H} 4$ acetylation by MOF, an acetyltransferase essential for dosage compensation in Drosophila. Mol Cell 5, 367-75.

Akhtar, A., Zink, D. and Becker, P. B. (2000). Chromodomains are proteinRNA interaction modules. Nature 407, 405-9.

Baek, S. H., Ohgi, K. A., Rose, D. W., Koo, E. H., Glass, C. K. and Rosenfeld, M. G. (2002). Exchange of N-CoR corepressor and Tip60 coactivator complexes links gene expression by NF-kappaB and beta-amyloid precursor protein. Cell 110, 55-67.

Bauer, A., Chauvet, S., Huber, O., Usseglio, F., Rothbacher, U., Aragnol, D., Kemler, R. and Pradel, J. (2000). Pontin52 and reptin52 function as antagonistic regulators of beta-catenin signalling activity. Embo $J$ 19, 6121-30.

Becker, P. B. and Horz, W. (2002). ATP-dependent nucleosome remodeling. Anпи Rev Biochem 71, 247-73.

Beissbarth, T. and Speed, T. P. (2004). GOstat: find statistically overrepresented Gene Ontologies within a group of genes. Bioinformatics 20, 1464-5.

Bellosta, P., Hulf, T., Balla Diop, S., Usseglio, F., Pradel, J., Aragnol, D. and Gallant, P. (2005). Myc interacts genetically with Tip48/Reptin and Tip49/Pontin to control growth and proliferation during Drosophila development. Proc Natl Acad Sci U $S$ A 102, 11799-804.

Bier, E. (2000). Drawing lines in the Drosophila wing: initiation of wing vein development. Curr Opin Genet Dev 10, 393-8.

Blair, S. S. (2007). Wing vein patterning in Drosophila and the analysis of intercellular signaling. Anпи Rev Cell Dev Biol 23, 293-319. 
Brand, A. H. and Perrimon, N. (1993). Targeted gene expression as a means of altering cell fates and generating dominant phenotypes. Development 118, 401-15.

Buscaino, A., Kocher, T., Kind, J. H., Holz, H., Taipale, M., Wagner, K., Wilm, M. and Akhtar, A. (2003). MOF-regulated acetylation of MSL-3 in the Drosophila dosage compensation complex. Mol Cell 11, 1265-77.

Cao, X. and Sudhof, T. C. (2001). A transcriptionally [correction of transcriptively] active complex of APP with Fe65 and histone acetyltransferase Tip60. Science 293, 115-20.

Carrozza, M. J., Utley, R. T., Workman, J. L. and Cote, J. (2003). The diverse functions of histone acetyltransferase complexes. Trends Genet 19, 321-9.

Ceol, C. J. and Horvitz, H. R. (2004). A new class of C. elegans synMuv genes implicates a Tip60/NuA4-like HAT complex as a negative regulator of Ras signaling. Dev Cell 6, 563-76.

Czermin, B., Melfi, R., McCabe, D., Seitz, V., Imhof, A. and Pirrotta, V. (2002). Drosophila enhancer of Zeste/ESC complexes have a histone H3 methyltransferase activity that marks chromosomal Polycomb sites. Cell 111, 185-96.

Diop, S. B., Bertaux, K., Vasanthi, D., Sarkeshik, A., Goirand, B., Aragnol, D., Tolwinski, N. S., Cole, M. D., Pradel, J., Yates, J. R., 3rd et al. (2008). Reptin and Pontin function antagonistically with PcG and TrxG complexes to mediate Hox gene control. EMBO Rep 9, 260-6.

Doyon, Y., Selleck, W., Lane, W. S., Tan, S. and Cote, J. (2004). Structural and functional conservation of the NuA4 histone acetyltransferase complex from yeast to humans. Mol Cell Biol 24, 1884-96.

Ebert, A., Lein, S., Schotta, G. and Reuter, G. (2006). Histone modification and the control of heterochromatic gene silencing in Drosophila. Chromosome Res 14, 377-92.

Eggert, H., Gortchakov, A. and Saumweber, H. (2004). Identification of the Drosophila interband-specific protein $\mathrm{Z} 4$ as a DNA-binding zinc-finger protein determining chromosomal structure. J Cell Sci 117, 4253-64.

Ehrenhofer-Murray, A. E. (2004). Chromatin dynamics at DNA replication, transcription and repair. Eur J Biochem 271, 2335-49.

Ehrenhofer-Murray, A. E., Rivier, D. H. and Rine, J. (1997). The role of Sas2, an acetyltransferase homologue of Saccharomyces cerevisiae, in silencing and ORC function. Genetics 145, 923-34. 
Fischle, W., Dequiedt, F., Fillion, M., Hendzel, M. J., Voelter, W. and Verdin, E. (2001). Human HDAC7 histone deacetylase activity is associated with HDAC3 in vivo. J Biol Chem 276, 35826-35.

Foglietti, C., Filocamo, G., Cundari, E., De Rinaldis, E., Lahm, A., Cortese, R. and Steinkuhler, C. (2006). Dissecting the biological functions of Drosophila histone deacetylases by RNA interference and transcriptional profiling. J Biol Chem 281, 17968-76.

Frank, S. R., Parisi, T., Taubert, S., Fernandez, P., Fuchs, M., Chan, H. M., Livingston, D. M. and Amati, B. (2003). MYC recruits the TIP60 histone acetyltransferase complex to chromatin. EMBO Rep 4, 575-80.

Gallant, P. (2007). Control of transcription by Pontin and Reptin. Trends Cell Biol 17, 187-92.

Gaughan, L., Brady, M. E., Cook, S., Neal, D. E. and Robson, C. N. (2001). Tip60 is a co-activator specific for class I nuclear hormone receptors. J Biol Chem 276, 46841-8.

Gause, M., Eissenberg, J. C., Macrae, A. F., Dorsett, M., Misulovin, Z. and Dorsett, D. (2006). Nipped-A, the Tra1/TRRAP subunit of the Drosophila SAGA and Tip60 complexes, has multiple roles in Notch signaling during wing development. Mol Cell Biol 26, 2347-59.

Gavaravarapu, S. and Kamine, J. (2000). Tip60 inhibits activation of CREB protein by protein kinase A. Biochem Biophys Res Commun 269, 758-66.

Godde, J. S. and Ura, K. (2008). Cracking the enigmatic linker histone code. $J$ Biochem 143, 287-93.

Gorrini, C., Squatrito, M., Luise, C., Syed, N., Perna, D., Wark, L., Martinato, F., Sardella, D., Verrecchia, A., Bennett, S. et al. (2007). Tip60 is a haplo-insufficient tumour suppressor required for an oncogene-induced DNA damage response. Nature 448, 1063-7.

Gortchakov, A. A., Eggert, H., Gan, M., Mattow, J., Zhimulev, I. F. and Saumweber, H. (2005). Chriz, a chromodomain protein specific for the interbands of Drosophila melanogaster polytene chromosomes. Chromosoma 114, 54-66.

Grewal, S. I. and Moazed, D. (2003). Heterochromatin and epigenetic control of gene expression. Science 301, 798-802.

Grienenberger, A., Miotto, B., Sagnier, T., Cavalli, G., Schramke, V., Geli, V., Mariol, M. C., Berenger, H., Graba, Y. and Pradel, J. (2002). The MYST 
domain acetyltransferase Chameau functions in epigenetic mechanisms of transcriptional repression. Curr Biol 12, 762-6.

Hammond, S. M., Bernstein, E., Beach, D. and Hannon, G. J. (2000). An RNA-directed nuclease mediates post-transcriptional gene silencing in Drosophila cells. Nature 404, 293-6.

Hanai, K., Furuhashi, H., Yamamoto, T., Akasaka, K. and Hirose, S. (2008). RSF governs silent chromatin formation via histone H2Av replacement. PLoS Genet 4 , e1000011.

Ikura, T., Ogryzko, V. V., Grigoriev, M., Groisman, R., Wang, J., Horikoshi, M., Scully, R., Qin, J. and Nakatani, Y. (2000). Involvement of the TIP60 histone acetylase complex in DNA repair and apoptosis. Cell 102, 463-73.

Kamakaka, R. T. and Biggins, S. (2005). Histone variants: deviants? Genes Dev 19, 295-310.

Kamine, J., Elangovan, B., Subramanian, T., Coleman, D. and Chinnadurai, G. (1996). Identification of a cellular protein that specifically interacts with the essential cysteine region of the HIV-1 Tat transactivator. Virology 216, 357-66.

Kao, H. Y., Downes, M., Ordentlich, P. and Evans, R. M. (2000). Isolation of a novel histone deacetylase reveals that class I and class II deacetylases promote SMRT-mediated repression. Genes Dev 14, 55-66.

Kesper, D. A., Stute, C., Buttgereit, D., Kreiskother, N., Vishnu, S., Fischbach, K. F. and Renkawitz-Pohl, R. (2007). Myoblast fusion in Drosophila melanogaster is mediated through a fusion-restricted myogenic-adhesive structure (FuRMAS). Dev Dyn 236, 404-15.

Kim, M. Y., Ann, E. J., Kim, J. Y., Mo, J. S., Park, J. H., Kim, S. Y., Seo, M. S. and Park, H. S. (2007). Tip60 histone acetyltransferase acts as a negative regulator of Notch1 signaling by means of acetylation. Mol Cell Biol 27, 6506-19.

Kimura, A., Umehara, T. and Horikoshi, M. (2002). Chromosomal gradient of histone acetylation established by Sas $2 p$ and Sir $2 p$ functions as a shield against gene silencing. Nat Genet 32, 370-7.

Kind, J., Vaquerizas, J. M., Gebhardt, P., Gentzel, M., Luscombe, N. M., Bertone, P. and Akhtar, A. (2008). Genome-wide analysis reveals MOF as a key regulator of dosage compensation and gene expression in Drosophila. Cell 133, 813-28. 
Kinner, A., Wu, W., Staudt, C. and Iliakis, G. (2008). Gamma-H2AX in recognition and signaling of DNA double-strand breaks in the context of chromatin. Nucleic Acids Res 36, 5678-94.

Kinoshita, A., Shah, T., Tangredi, M. M., Strickland, D. K. and Hyman, B. T. (2003). The intracellular domain of the low density lipoprotein receptor-related protein modulates transactivation mediated by amyloid precursor protein and Fe65. $J$ Biol Chem 278, 41182-8.

Kinoshita, A., Whelan, C. M., Berezovska, O. and Hyman, B. T. (2002). The gamma secretase-generated carboxyl-terminal domain of the amyloid precursor protein induces apoptosis via Tip60 in H4 cells. J Biol Chem 277, 28530-6.

Kornberg, R. D. (1974). Chromatin structure: a repeating unit of histones and DNA. Science 184, 868-71.

Kramer, J. M. and Staveley, B. E. (2003). GAL4 causes developmental defects and apoptosis when expressed in the developing eye of Drosophila melanogaster. Genet Mol Res 2, 43-7.

Kusch, T., Florens, L., Macdonald, W. H., Swanson, S. K., Glaser, R. L., Yates, J. R., 3rd, Abmayr, S. M., Washburn, M. P. and Workman, J. L. (2004). Acetylation by Tip60 is required for selective histone variant exchange at DNA lesions. Science 306, 2084-7.

Lange, B. M., Rebollo, E., Herold, A. and Gonzalez, C. (2002). Cdc37 is essential for chromosome segregation and cytokinesis in higher eukaryotes. Embo J 21, 5364-74.

Legube, G., Linares, L. K., Tyteca, S., Caron, C., Scheffner, M., ChevillardBriet, M. and Trouche, D. (2004). Role of the histone acetyl transferase Tip60 in the p53 pathway. J Biol Chem 279, 44825-33.

Luger, K., Mader, A. W., Richmond, R. K., Sargent, D. F. and Richmond, T. J. (1997). Crystal structure of the nucleosome core particle at 2.8 A resolution. Nature 389, 251-60.

Mavrich, T. N., Jiang, C., Ioshikhes, I. P., Li, X., Venters, B. J., Zanton, S. J., Tomsho, L. P., Qi, J., Glaser, R. L., Schuster, S. C. et al. (2008). Nucleosome organization in the Drosophila genome. Nature 453, 358-62.

McBryant, S. J., Adams, V. H. and Hansen, J. C. (2006). Chromatin architectural proteins. Chromosome Res 14, 39-51. 
McGuire, S. E., Roman, G. and Davis, R. L. (2004). Gene expression systems in Drosophila: a synthesis of time and space. Trends Genet 20, 384-91.

Meijsing, S. H. and Ehrenhofer-Murray, A. E. (2001). The silencing complex SAS-I links histone acetylation to the assembly of repressed chromatin by CAF-I and Asf1 in Saccharomyces cerevisiae. Genes Dev 15, 3169-82.

Mendjan, S. and Akhtar, A. (2007). The right dose for every sex. Chromosoma 116, $95-106$.

Miotto, B., Sagnier, T., Berenger, H., Bohmann, D., Pradel, J. and Graba, Y. (2006). Chameau HAT and DRpd3 HDAC function as antagonistic cofactors of JNK/AP-1-dependent transcription during Drosophila metamorphosis. Genes Dev 20, $101-12$.

Mottus, R., Sobel, R. E. and Grigliatti, T. A. (2000). Mutational analysis of a histone deacetylase in Drosophila melanogaster: missense mutations suppress gene silencing associated with position effect variegation. Genetics 154, 657-68.

Muller, H., Fogeron, M. L., Lehmann, V., Lehrach, H. and Lange, B. M. (2006). A centrosome-independent role for gamma-TuRC proteins in the spindle assembly checkpoint. Science $\mathbf{3 1 4}, 654-7$.

Muller, J., Hart, C. M., Francis, N. J., Vargas, M. L., Sengupta, A., Wild, B., Miller, E. L., O'Connor, M. B., Kingston, R. E. and Simon, J. A. (2002). Histone methyltransferase activity of a Drosophila Polycomb group repressor complex. Cell 111, 197-208.

Negeri, D., Eggert, H., Gienapp, R. and Saumweber, H. (2002). Inducible RNA interference uncovers the Drosophila protein $\mathrm{Bx} 42$ as an essential nuclear cofactor involved in Notch signal transduction. Mech Dev 117, 151-62.

Patel, J. H., Du, Y., Ard, P. G., Phillips, C., Carella, B., Chen, C. J., Rakowski, C., Chatterjee, C., Lieberman, P. M., Lane, W. S. et al. (2004). The cMYC oncoprotein is a substrate of the acetyltransferases hGCN5/PCAF and TIP60. Mol Cell Biol 24, 10826-34.

Qi, D., Jin, H., Lilja, T. and Mannervik, M. (2006). Drosophila Reptin and other TIP60 complex components promote generation of silent chromatin. Genetics 174, 241-51.

Reifsnyder, C., Lowell, J., Clarke, A. and Pillus, L. (1996). Yeast SAS silencing genes and human genes associated with AML and HIV-1 Tat interactions are homologous with acetyltransferases. Nat Genet 14, 42-9. 
Robert, F., Hardy, S., Nagy, Z., Baldeyron, C., Murr, R., Dery, U., Masson, J. Y., Papadopoulo, D., Herceg, Z. and Tora, L. (2006). The transcriptional histone acetyltransferase cofactor TRRAP associates with the MRN repair complex and plays a role in DNA double-strand break repair. Mol Cell Biol 26, 402-12.

Robertson, H. M., Preston, C. R., Phillis, R. W., Johnson-Schlitz, D. M., Benz, W. K. and Engels, W. R. (1988). A stable genomic source of P element transposase in Drosophila melanogaster. Genetics 118, 461-70.

Sambrook, J., Fritsch, E. F. and Sambrook, J. (1989). Molecular cloning: a laboratory manual. Cold Spring Harbor, N.Y.: Cold Spring Harbor Laboratory Press.

Sanjuan, R. and Marin, I. (2001). Tracing the origin of the compensasome: evolutionary history of DEAH helicase and MYST acetyltransferase gene families. $\mathrm{Mol}$ Biol Evol 18, 330-43.

Sapountzi, V., Logan, I. R. and Robson, C. N. (2006). Cellular functions of TIP60. Int J Biochem Cell Biol 38, 1496-509.

Schneider, I. (1972). Cell lines derived from late embryonic stages of Drosophila melanogaster. J Embryol Exp Morphol 27, 353-65.

Scott, E. K., Lee, T. and Luo, L. (2001). enok encodes a Drosophila putative histone acetyltransferase required for mushroom body neuroblast proliferation. Curr Biol 11, 99-104.

Shia, W. J., Li, B. and Workman, J. L. (2006). SAS-mediated acetylation of histone H4 Lys 16 is required for H2A.Z incorporation at subtelomeric regions in Saccharomyces cerevisiae. Genes Dev 20, 2507-12.

Squatrito, M., Gorrini, C. and Amati, B. (2006). Tip60 in DNA damage response and growth control: many tricks in one HAT. Trends Cell Biol 16, 433-42.

Suka, N., Luo, K. and Grunstein, M. (2002). Sir2p and Sas2p opposingly regulate acetylation of yeast histone $\mathrm{H} 4$ lysine 16 and spreading of heterochromatin. Nat Genet 32, 378-83.

Sumioka, A., Nagaishi, S., Yoshida, T., Lin, A., Miura, M. and Suzuki, T. (2005). Role of 14-3-3gamma in FE65-dependent gene transactivation mediated by the amyloid beta-protein precursor cytoplasmic fragment. J Biol Chem 280, 42364-74.

Swaminathan, J., Baxter, E. M. and Corces, V. G. (2005). The role of histone $\mathrm{H} 2 \mathrm{Av}$ variant replacement and histone $\mathrm{H} 4$ acetylation in the establishment of Drosophila heterochromatin. Genes Dev 19, 65-76. 
Sykes, S. M., Mellert, H. S., Holbert, M. A., Li, K., Marmorstein, R., Lane, W. S. and McMahon, S. B. (2006). Acetylation of the p53 DNA-binding domain regulates apoptosis induction. Mol Cell 24, 841-51.

Tang, Y., Luo, J., Zhang, W. and Gu, W. (2006). Tip60-dependent acetylation of p53 modulates the decision between cell-cycle arrest and apoptosis. Mol Cell 24, 827-39.

Taubert, S., Gorrini, C., Frank, S. R., Parisi, T., Fuchs, M., Chan, H. M., Livingston, D. M. and Amati, B. (2004). E2F-dependent histone acetylation and recruitment of the Tip60 acetyltransferase complex to chromatin in late G1. Mol Cell Biol 24, 4546-56.

Turner, B. M. (2002). Cellular memory and the histone code. Cell 111, $285-91$.

Utley, R. T. and Cote, J. (2003). The MYST family of histone acetyltransferases. Curr Top Microbiol Immunol 274, 203-36.

Wallrath, L. L. and Elgin, S. C. (1995). Position effect variegation in Drosophila is associated with an altered chromatin structure. Genes Dev 9, 1263-77.

Workman, J. L. and Kingston, R. E. (1998). Alteration of nucleosome structure as a mechanism of transcriptional regulation. Annu Rev Biochem 67, 545-79.

Wu, C. H., Yamaguchi, Y., Benjamin, L. R., Horvat-Gordon, M., Washinsky, J., Enerly, E., Larsson, J., Lambertsson, A., Handa, H. and Gilmour, D. (2003). NELF and DSIF cause promoter proximal pausing on the hsp70 promoter in Drosophila. Genes Dev 17, 1402-14.

Xiao, H., Chung, J., Kao, H. Y. and Yang, Y. C. (2003). Tip60 is a corepressor for STAT3. J Biol Chem 278, 11197-204.

Zhang, L., Eugeni, E. E., Parthun, M. R. and Freitas, M. A. (2003). Identification of novel histone post-translational modifications by peptide mass fingerprinting. Chromosoma 112, 77-86.

Zhu, X., Singh, N., Donnelly, C., Boimel, P. and Elefant, F. (2007). The cloning and characterization of the histone acetyltransferase human homolog DmellTIP60 in Drosophila melanogaster: DmellTIP60 is essential for multicellular development. Genetics 175, 1229-40. 


\section{Danksagung}

Mein großer Dank gilt Frau Prof. Ann Ehrenhofer-Murray für die Möglichkeit, dieses spannende Projekt in ihrem Labor bearbeiten $\mathrm{zu}$ dürfen und ihre kontinuierliche Unterstützung bei der Bearbeitung dieses Projektes. Des weiteren danke ich Prof. Harald Saumweber für die Einführung in die Arbeit mit Drosophila. Als besonders unterstützend und bereichernd habe ich die Zusammenarbeit mit Dr. Ludger KleinHitpaß und mit Prof. Daniel Hoffmann bei der Analyse und anschließenden bioinformatischen Auswertung der Microarray-Daten empfunden.

Allen jetzigen und früheren Mitarbeitern des Ehrenhofer-Murray Labors danke ich für angeregte Diskussionen und große Kollegialität.

Ich danke meinen Eltern, Judith Schrodt, Franziska Seifert und Carina Hoeft. 


\section{Lebenslauf}

Persönliche Daten

Corinna Schirling

Adresse

Busehofstr. 71

45144 Essen

Email

corinna.schirling@uni-duisburg-essen.de

Geburtsdatum, -ort

11. Oktober 1977, Heilbronn a.N.

Ausbildung

$1987-1994$

Hohenlohe Gymnasium Öhringen

$1994-1997$

Agrarwissenschaftliches Gymnasium Öhringen

Juni 1997

allgemeine Hochschulreife

WS 1997/98 - WS 1998/99

Studium der Biochemie, Universität Regensburg

SS 1999 - WS 2003/2004

Studium der Biochemie, Freie Universität Berlin

18. 11.2003

Diplom (Biochemie)

\section{Dissertation}

1. 12. 2003 bis 31.12 .2004

wiss. Mitarbeiterin

MPI für Molekulare Genetik, Berlin

Arbeitsgruppe Ehrenhofer-Murray

Beginn der Dissertation

1. 1.2005 bis 31.7 .2005

wiss. Mitarbeiterin

Justus-Liebig-Universität Giessen

Arbeitsort: Berlin, Fortsetzung der Dissertation unter Anleitung von Frau Ehrenhofer-Murray

1. 8. 2005 - gegenwärtig

wiss. Mitarbeiterin

Universität Duisburg - Essen

Fortsetzung der Dissertation am neu berufenen

Lehrstuhl für Genetik bei Frau Prof. Dr. EhrenhoferMurray 